NASA-CR-203015

$N \not$

ICARUS 113, 232-266 (1995)

\title{
An Analysis of Neptune's Stratospheric Haze Using High-Phase-Angle Voyager Images
}

\author{
JuLianne I. Moses \\ Lunar and Planetary Institute, 3600 Bay Area Boulevard, Houston, Texas 77058-1113 \\ E-mail: moses@lpi.jsc.nasa.gov \\ Kathy Rages \\ Space Physics Research Institute, Sunnyvale, California 94087
}

AND

James B. Pollack $\dagger$

NASA Ames Research Center, Moffen Field, California 94035

Received May 18, 1994; revised September 26, 1994

\begin{abstract}
We have inverted high-phase-angle Voyager images of Neptune to determine the atmospheric extinction coefficient as a function of altitude and the scattering phase function at a reference altitude. Comparisons between theoretical models and observations help separate the contributions from molecular Rayleigh and aerosol scattering and help determine the variation of the aerosol size, concentration, and scattering properties with altitude. Further comparisons between models and data allow us to place constraints on the location and composition of the hazes, the concentration and downward flux of certain condensible hydrocarbon gases, the eddy diffusion coefficient in the lower stratosphere, and the thermal profile in parts of Neptune's stratosphere. We find that a distinct stratospheric haze layer exists near $12_{-1}^{+1} \mathrm{mbar}$ in Neptune's lower stratosphere, most probably due to condensed ethane. The derived stratospheric haze production rate of $1.0_{-0.3}^{+0.2} \times 10^{-15} \mathrm{~g} \mathrm{~cm}^{-2} \mathrm{sec}^{-1}$ is substantially lower than photochemical model predictions. Evidence for hazes at higher altitudes also exists. Unlike the situation on Uranus, large particles $\mathbf{( 0 . 0 8 -}$ $0.11 \mu \mathrm{m}$ ) may be present at high altitudes on Neptune (e.g., near 0.5 mbar), well above the region in which we expect the major hydrocarbon species to condense. Near $28 \mathrm{mbar}$, the mean particle size is about $0.13_{-0.02}^{+0.02} \mu \mathrm{m}$ with a concentration of $5_{-3}^{+3}$ particles $\mathrm{cm}^{-3}$. The cumulative haze extinction optical depth above 15 mbar in the clear filter is $\sim 3 \times 10^{-3}$, and much of this extinction is due to scattering rather than absorption; thus, if our limb-scan sites are typical, the hazes cannot account for the stratospheric temperature inversion on Neptune and may not contribute signifcantly to atmospheric heating. We compare the imaging results with the results from other observations, including those of the Voyager Photopolarimeter Subsystem, and discuss differences between Neptune and Uranus. 1995 Academic Press, Inc.
\end{abstract}

\footnotetext{
† Deceased.
}

\section{INTRODUCTION}

None of the planetary atmospheres in our solar system are perfectly clean; all contain dust, haze, or cloud particles that obscure our view and can profoundly affect physics and chemistry on the planet. Aerosol particles can modify the scattering properties, thermal structure, energy balance, dynamics, and photochemistry of an atmosphere; thus, a good understanding of the particle characteristics and behavior is essential to understanding some of the basic underlying properties of the planetary system. In the case of the outer planets (Jupiter, Saturn, Uranus, and Neptune), optically thick clouds develop from the condensation of major gas species in the troposphere while thinner high altitude hazes form when ultraviolet radiation or energetic particles break down atmospheric gases to form less volatile species. Particles may also form from an influx of extraplanetary debris.

Uranus and Neptune are so cold that most of the major gas molecules other than $\mathrm{H}_{2}$ condense far below the tropopause and do not affect photochemistry or haze formation in the upper atmosphere. Methane is an exception to this rule. Methane is abundant, yet volatile enough that it will condense in the upper troposphere and still have a significant mixing ratio at the tropopause cold trap; thus, it will diffuse or be dynamically injected into the upper atmosphere, become dissociated by ultraviolet radiation or charged particles, and instigate the photochemistry on Uranus and Neptune. The methane photolysis products react with themselves and with hydrogen to form a host of hydrocarbon species. These species diffuse downward into the colder lower stratosphere where 


\section{$\therefore \quad \cdots$}


some can condense to form hazes (see reviews by West et al. 1991, Atreya et al. 1991, and Baines et al. 1994).

The relative simplicity of this single-species-driven photochemistry has made Uranus and Neptune attractive targets for theoretical modeling. Early theoretical studies of photochemistry and haze formation on Uranus (Atreya and Ponthieu 1983, Atreya and Romani 1985) and Neptune (Romani and Atreya 1988, 1989) have shown that methane photolysis products such as ethane and acetylene should condense in the stratosphere. Often, these authors predict both the altitude levels at which the hydrocarbons should condense and the total column production rate of stratospheric aerosols-predictions that can be tested directly by appropriate spacecraft or ground-based observations. The data from Voyager 2's encounter with Uranus (J. Geophys. Res. special issue, 92, December 30, 1987 and Science special issue, 233, July 4,1986 ) caused some revisions in the photochemical models (Summers and Strobel 1989, Atreya et al. 1991) and gave us some valuable information concerning the methane cycle on the outer planets, but did not drastically change the simple qualitative picture of methanedriven photochemistry and haze formation on Uranus and Neptune.

Quantitatively, however, the Voyager observations of the hazes on Uranus produced some mild surprises. For instance, both the observed altitude dependence of the particle concentration (e.g., the relative importance of the different condensible hydrocarbon species) and the total inferred haze production rate seemed to be different than expected (Pollack et al. 1987, Rages et al. 1991, Atreya et al. 1991). No great note of these discrepancies was taken since the differences appeared to be within model uncertainties. However, recent analyses of the Voyager data from the Neptune encounter (J. Geophys. Res. special issue, 96, December 30, 1991; Science special issue, 246, December 15, 1989; Pryor et al. 1992; Yelle et al. 1993), of ground-based visible and infrared observations of Neptune (Baines and Hammel 1994, Orton et al. 1992), and of the Neptune Voyager imaging data discussed in this paper suggest that we do not fully understand the processes of photochemistry and haze formation on Uranus and Neptune. As will be discussed later in this paper, these recent observations of the stratospheric hazes have produced some unexpected results that have provided us with some valuable insights and further puzzles into the formation of hazes on the outer planets.

In this paper, we extend the preliminary investigation reported by Smith et al. (1989) and analyze some of the high-phase-angle images acquired by the Voyager 2 Imaging Subsystem (ISS) during the Neptune encounter. Our analysis procedure is similar to that used by Pollack et al. (1987) in their examination of the high-phase-angle data from Uranus. High-phase-angle images are particu- larly useful for determining properties of stratospheric scattering particles. Haze particles typically have phase functions that are sharply peaked at low scattering angles (high phase angles); thus, it is easier to separate the contributions from forward-scattering hazes and the symmetric Rayleigh-scattering gas molecules in high-phaseangle images. Also, multiple scattering occurs less frequently at high phase angles, allowing the single-scattering properties of the stratospheric hazes to be readily inferred from the images. Furthermore, the excellent spatial resolution of the high-phase-angle images enables us to readily separate contributions from different altitudes in the stratosphere.

Important complements to our imaging analysis are provided by several theoretical models designed to simulate the observed extinction in the high-phase-angle images. We construct photochemical models to predict the composition, condensation levels, and production rates of hydrocarbon hazes in Neptune's stratosphere. These results are then used as input to an aerosol-dynamics model that helps us predict the concentration and size distribution of the aerosols as a function of altitude. With a Rayleigh and Mie single-scattering code, we then compare the predicted extinction from this aerosol distribution with observations. This comparison allows us to constrain several physical parameters of interest in the stratospheric haze layers. The multiwavelength nature of the data set ensures that we can place relatively tight constraints on these parameters, and comparisons with the high-phase-angle observations from the Voyager Photopolarimeter Subsystem (PPS) experiment (Pryor et al. 1992) - observations taken in the same latitude region as the ISS high-phase-angle images-give us more insight into the formation and evolution of the stratospheric aerosol layers on Neptune.

In the following sections, we describe our image-processing techniques, inversion algorithms, and theoretical models. Next, we present our inversion results for $29^{\circ} \mathrm{S}$ latitude and discuss a number of issues that arise during comparisons between the observations and theoretical models. We then discuss the primary constraints on the physical properties of the stratospheric hazes that have been determined from the model-data comparisons and discuss the sensitivity of our theoretical models to free parameters. Finally, we present our principal conclusions.

\section{VOYAGER IMAGES}

A series of wide- and narrow-angle camera images of Neptune's illuminated crescent were obtained in August 1989 after Voyager 2's closest approach with the planet. We have selected five of the highest phase-angle images to use in our analysis of the Neptune stratospheric haze. 
TABLE I

Voyager Image Characteristics

\begin{tabular}{clccc}
\hline $\begin{array}{c}\text { FDS } \\
\text { count }\end{array}$ & $\begin{array}{c}\text { Camera } \\
\text { and filter }\end{array}$ & $\begin{array}{c}\text { Phase angle } \\
\left({ }^{\circ}\right)\end{array}$ & $\begin{array}{c}\text { Resolution } \\
(\mathrm{km} / \text { line pair })\end{array}$ & $\begin{array}{c}\text { Latitude range } \\
\left({ }^{\circ}\right)\end{array}$ \\
\hline 11392.23 & NA clear & 158.3 to 158.6 & 2.02 & -28.2 to -30.6 \\
11392.13 & WA violet & 159 to 161 & 14.0 & -22.0 to -38.8 \\
11392.17 & WA blue & 158 to 161 & 14.5 & -24.1 to -40.3 \\
11392.11 & WA green & 159 to 162 & 13.8 & -22.0 to -35.7 \\
11392.15 & WA orange & 158 to 162 & 14.3 & -22.0 to -40.3 \\
\hline
\end{tabular}

a Since the point-spread function of the Voyager cameras is $\sim 1.67$ pixels ( $S$. A. Collins, private communication), the resolution is given per line pair (e.g., 2 pixels) rather than per pixel.

The quality of the data set is excellent. Four wide-angle camera images at $160^{\circ}$ phase angle exhibit a spatial resolution of better than $15 \mathrm{~km} /$ line pair while a narrow-angle clear-filter image at a phase angle of $158^{\circ}$ exhibits a resolution of about $2 \mathrm{~km} /$ line pair-much better resolution than has ever been obtained for high-phase-angle images of any of the giant planets (note that atmospheric scale heights range from $17 \mathrm{~km}$ in the lower stratosphere to 50 $\mathrm{km}$ in the middle stratosphere). All five images were taken within 10 min of each other and are centered near the same planetary latitude and longitude. Table I sum- marizes several characteristics of these images; all latitudes quoted throughout the paper are in planetographic coordinates. We have processed the images in the manner described in detail in Rages et al. (1991) and Pollack et al. (1987). The goal of the image processing is to determine the specific intensity $(I / F)$ and atmospheric extinction coefficient as a function of planetary radius across Neptune's limb. See Appendix A for further details.

To help orient our limb-scan sites in relation to prominent albedo features on the planet, we have included an image taken approximately $11 \mathrm{~h}$ before our high-phaseangle images (see Fig. 1). The image in Fig. 1a was taken with the wide-angle camera in a methane filter ( $\mathrm{MeU}$, efficient wavelength $0.543 \mu \mathrm{m}$ ). The image has not been processed beyond simple pointing and stretch corrections; it is included mainly to show where our limb sites appear in relation to the Great Dark Spot (GDS) and Bright Companion (BC). The GDS and $\mathrm{BC}$ are visible in the upper left of Fig. 1a while our limb-scan sites are on the center right (see Fig. $1 \mathrm{~b}$ for a comparison grid).

Our principal latitude region of $29^{\circ}-30^{\circ} \mathrm{S}$ is only a degree or so south of the $\mathrm{BC}$ and is located nearly $90^{\circ}$ east of the $\mathrm{BC}$ at the time our high-phase-angle images were taken. The latitude region we examine is relatively free of bright albedo features that can help determine wind speeds (Limaye and Sromovsky 1991), suggesting that
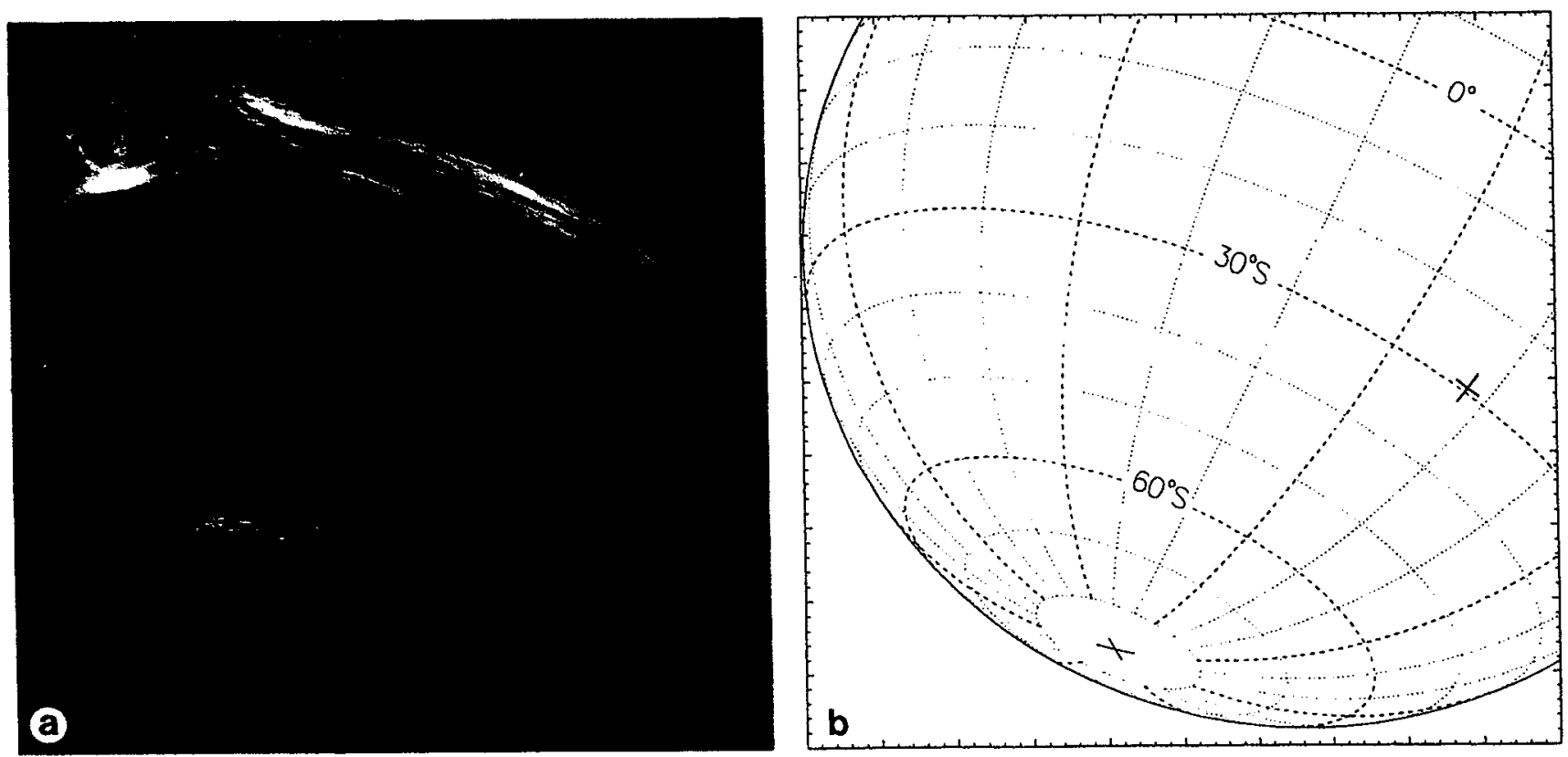

FIG. 1. (a) A methane-band (MeU) image of Neptune (FDS 11378.06) taken $11.25 \mathrm{hr}$ before our narrow-angle camera clear-filter image. The Great Dark Spot and Bright Companion are visible in the upper left of the image. (b) A companion grid shows the location of our NA-camera limbscan site at that time; the position is marked with a cross at the right side of the grid. 
this latitude region might be relatively free of cloud or haze particles at the altitudes responsible for the bright streaks emanating from the GDS. On the other hand, ultraviolet PPS images (Lane et al. 1989) and visible ISS images (Smith et al. 1989) indicate that this latitude region is within the southern end of an equatorial band that appears bright in the visible and dark in the ultraviolet, suggesting the prevalence of UV-absorbing and visiblescattering aerosols at this location. In any case, we want to point out that stratospheric hazes in our latitude region may be affected by the close proximity of the GDS, which has clearly influenced atmospheric activity at the time of the Voyager encounter.

\section{ATMOSPHERIC MODELS}

In order to fully interpret the Voyager imaging data, we have developed several theoretical models whose ultimate purpose is to reproduce the observed extinction in the high-phase-angle Neptune images. First of all, we have created a hydrostatic model atmosphere for Neptune to construct an accurate picture of the background gas composition and density variation with altitude. This hydrostatic model establishes the background atmosphere for both the photochemistry and aerosol-dynamics models and forms the basis for the Rayleigh-scattering calculation used in both the data inversion and the forward-modeling scattering routines. Secondly, we have constructed a one-dimensional photochemical model that allows us to predict the abundance, downward flux, and condensation levels of potentially condensible hydrocarbon species in the Neptune stratosphere. The results from this photochemical model are used as input to the third model, a one-dimensional aerosol-dynamics (cloudmicrophysics) model that follows the formation and evolution of the stratospheric aerosol particles. This aerosoldynamics model allows us to predict the concentration, size distribution, and other physical properties of the aerosols as a function of altitude on Neptune. Finally, we use a Mie- and Rayleigh-scattering program to take the output from the aerosol-dynamics routine and calculate the atmospheric and aerosol extinction as a function of altitude that would result from the predicted aerosol distribution. The model predictions are then compared directly with the Voyager observations.

\subsection{Background Hydrostatic Atmosphere}

In order to make meaningful comparisons with observations, the background atmosphere developed for our theoretical models must mirror that of the actual atmosphere. Unfortunately, our knowledge of Neptune's thermal structure, planetary shape, and variation of mean molecular mass with altitude is incomplete; therefore,

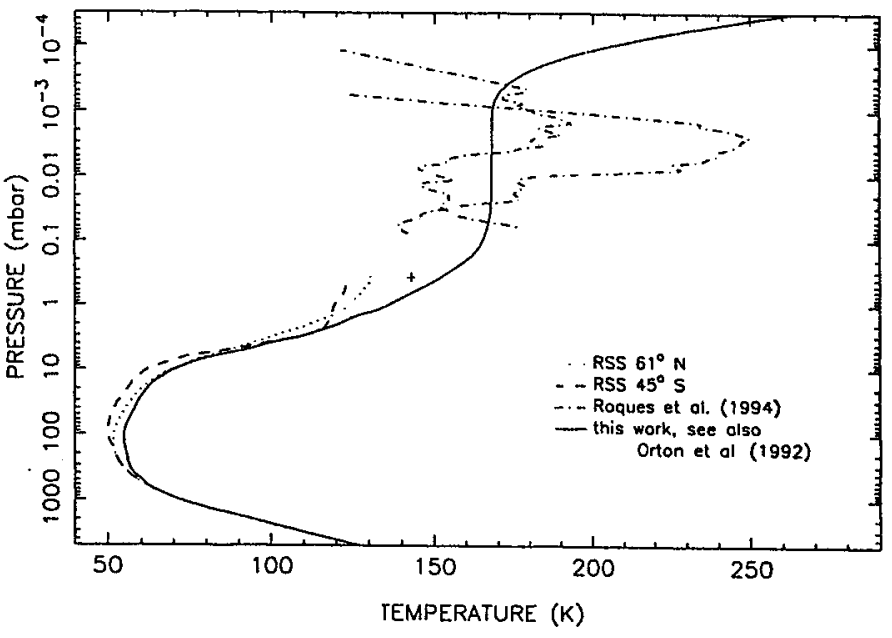

FIG. 2. Temperature profile for Neptune. The solid line depicts a global average profile as suggested by Orton et al. (1992) and is used throughout this paper. The dotted and dashed lines are the results from the RSS occultation experiments (Lindal 1992) for $61^{\circ} \mathrm{N}$ and $45^{\circ} \mathrm{S}$ latitude, respectively, and the dot-dash lines represent two results from the June 7, 1989 stellar occultation (immersion) of Neptune as observed at the ESO and Pic du Midi (Roques et al. 1994). The information from the observation of a central flash in the August 20,1985 occultation (Hubbard et al. 1987) is represented by a cross. All results are for an assumed helium abundance of $19 \%$.

our derived atmospheric density profile is somewhat uncertain. We have attempted to piece together all the available observations to develop a background atmosphere that closely resembles reality. The thermal profile adopted in our models is shown in Fig. 2. Note that we have adopted a thermal profile that is warmer than that derived for the RSS occultation sites in Neptune's troposphere and lower stratosphere. Conrath et al. (1991a) and Orton et al. (1992) demonstrate that the latitudes probed by the RSS experiment appear to be several degrees colder than most regions of the planet. Details of our adopted thermal profile and of the other parameters required for the development of our hydrostatic model Neptune atmosphere are given in Appendix B. The hydrogen number density profile resulting from the temperature profile and planetary characteristics discussed in Appendix B is shown in Fig. 3.

\subsection{Photochemistry}

In the second step of our forward modeling of Neptune's stratospheric hazes, we use a photochemical model to make predictions concerning the downward flux and condensation levels of the hydrocarbon species. We develop a one-dimensional model of hydrocarbon photochemistry using the Caltech chemical kinetics and diffusion code described in Allen et al. (1981). The reaction list, rate constants, quantum yields, and molecular diffu- 


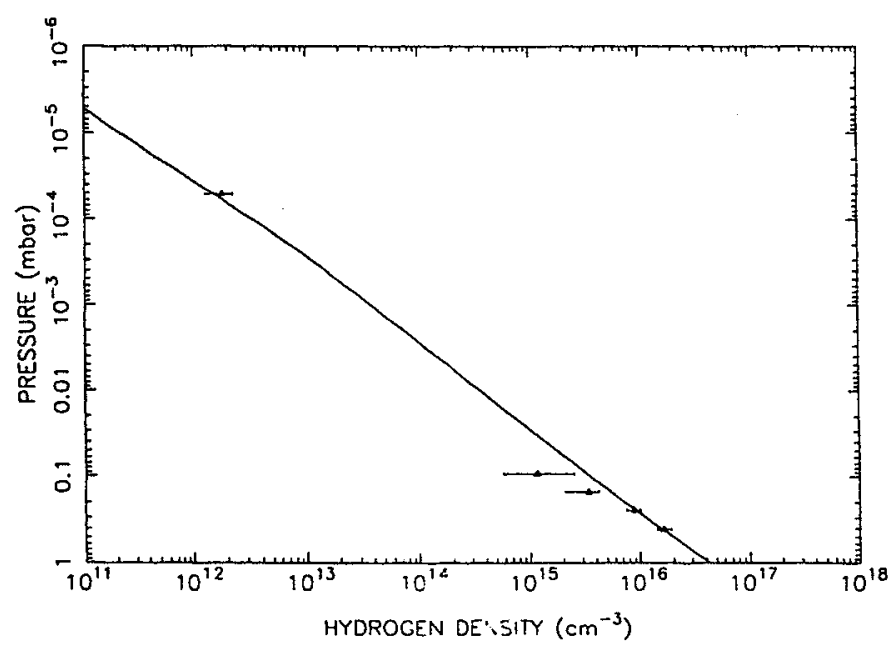

FIG. 3. The hydrogen density in our model (at $49^{\circ}$ latitude) using the temperature profile from Fig. 2. The observations (triangles with error bars) are from the egress UVS solar occultation experiment (Yelle et al. 1993, Fig. 8f).

sion coefficients are almost identical to those used in the Jupiter model of Gladstone et al. (1994), with minor changes as discussed by Moses (1991). Some recently reported reaction rates and dissociation pathways are not included. For example, Mordaunt et al. (1993) suggest that methane photolysis occurs in large part by a pathway not considered in this paper. Preliminary calculations indicate that the Mordaunt et al. photodissociation yields may reduce slightly but not otherwise affect the production rate of higher-order hydrocarbons such as $\mathrm{C}_{2} \mathrm{H}_{2}$ and $\mathrm{C}_{2} \mathrm{H}_{6}$ in outer-planetary atmospheres (Lee et al. 1993, Gladstone et al. 1994).

The model only includes hydrocarbon species (and helium) and does not include condensation; thus, the results for the concentrations of the individual species are approximate. On Neptune, eddy diffusion in the stratosphere is strong enough to carry methane up to relatively high altitudes. The condensible hydrocarbon gases are produced by methane photolysis (and subsequent chemical reactions) at relatively high altitudes, well above the low-temperature condensation regions; thus, little in situ aerosol production takes place as the gases are formed. Instead, the gases diffuse downward to the lower stratosphere until they eventually become supersaturated and condense. Our neglect of condensation in the photochemical model will therefore not affect the species abundances except within the condensation regions themselves (where the gas abundances will be depleted due to condensation) and within a scale height or so above the condensation regions (where the atmosphere to a lesser degree still "feels" the effects of condensation, cf. Romani and Atreya 1988, 1989). If our adopted eddy diffusion profile is correct, then the predictions for the total condensation flux should also be fairly accurate even if the photochemistry is incomplete because most of the carbon atoms that are liberated from methane by dissociation end up in condensible species.

The diffusion and chemical loss time scales for the major haze-forming species in Neptune's lower stratosphere are longer than a few years; thus, we use solar flux values that are intermediate between sunspot maximum and minimum conditions. The specific value (at $1 \mathrm{AU}$ ) for the $\mathrm{H}$ Lyman $\alpha$ flux that we use in $3.32 \times 10^{11}$ photons $\mathrm{cm}^{-2}$ $\mathrm{sec}^{-1}$ in a $1-\AA$ interval centered at $1215.7 \AA$. This value is slightly higher than average for the recent solar cycle 22 . Neptune's short rotational period of $16.1 \mathrm{hr}$ also inspires us to take diurnal averages. However, since Neptune's orbital period of 165 years is much longer than the lifetimes of these species, we do not take seasonal averages, but examine the photochemistry at the relevant season occurring at the time of the Voyager encounter. The contribution to the flux from solar radiation scattered from $\mathrm{H}$ atoms in the local interstellar medium (LISM) is nontrivial and is included in the calculations (see Moses 1991). Since we have little information concerning this LISM source, we use the value measured directly by the UVS instrument on Voyager during the 1989 Neptune encounter $\left(2.7 \times 10^{7}\right.$ photons $\mathrm{cm}^{-2} \mathrm{sec}^{-1}$ sterad $^{-1}$ at $30 \mathrm{AU}$, Broadfoot et al. 1989). Since this source should also vary with solar cycle, the value that we adopt is probably higher than average. As a result we may overpredict "average" ethane fluxes by a factor of $\leqslant 15 \%$. Moses (1991) provides a more detailed discussion of the solar and LISM spectrum used in this model and of the sensitivity of the hydrocarbon mixing ratios to the ultraviolet flux. The photochemical model calculations were performed at $49^{\circ} \mathrm{S}$ latitude for comparison with the UVS occultation results and at $30^{\circ} \mathrm{S}$ latitude to set up conditions relevant to the high-phase-angle images.

If the observed zonal winds on Neptune (e.g., Smith et al. 1989, Hammel et al. 1989, Limaye and Sromovsky 1991) are maintained in steady state by the Coriolis force acting on the meridional winds and if the frictional damping time in Neptune's lower stratosphere is comparable to the atmospheric radiative relaxation time, which is comparable to the planet's orbital period (as is suggested by Conrath et al. 1991a), then meridional wind speeds in Neptune's lower stratosphere should be of order $0.1 \mathrm{~mm}$ $\mathrm{sec}^{-1}$. In the course of a full Neptune year, stratospheric air parcels at $30^{\circ}$ latitude would move only a few degrees in latitude, and latitudinal exchange of aerosols should be minimal. Zonal wind speeds, on the other hand, are much stronger, and we would expect an air parcel to travel around the planet horizontally in the east-west direction in a few days or weeks. Thus, fewer longitudinal variations in the stratospheric haze properties should exist, 
although some variations may be possible, and indeed may even be visible if the bright features in the groundbased and Voyager methane-band images (e.g., Hammel 1989, Smith et al. 1989) extend to the stratosphere. Both of these traits of slow latitudinal mixing and rapid longitudinal mixing suggest that diurnally averaged one-dimensional models should provide reasonable representations of Neptune's stratosphere, at least as far as the aerosols are concerned.

The photochemical scheme we use is known to have problems reproducing the hydrocarbon observations. At this time, it is not clear whether the problems are due to the photochemical scheme itself, to the adopted eddy diffusion profile in Neptune's stratosphere, to real spatial and/or temporal differences in Neptune's stratosphere, or to uncertainties in the observational analyses. One perennial problem is that the photochemical scheme underestimates the $\mathrm{C}_{2} \mathrm{H}_{6} / \mathrm{C}_{2} \mathrm{H}_{2}$ ratio in all the outer planets, especially on Neptune (e.g., Moses 1991, Allen et al. 1992, Bézard et al. 1991, Romani et al. 1993). The problem could be that the reaction rates we use, which are often measured only at room temperature, are not appropriate to the cold temperatures in the outer planets. Additionally, we may be neglecting minor species (like oxygen and/or nitrogen compounds) that might have a significant effect on the photochemistry of the outer planets. If these additional species cause net destruction of hydrocarbon compounds such as $\mathrm{C}_{2} \mathrm{H}_{2}$ but do not form condensible species in the process, then the net aerosol production rate predicted from our models might be an upper limit. On the other hand, the problem with the $\mathrm{C}_{2} \mathrm{H}_{6} / \mathrm{C}_{2} \mathrm{H}_{2}$ ratio might be due to the failure of conventional models of the eddy diffusion coefficient as applied to outer planetary atmospheres rather than due to any problems with the chemistry. Romani et al. (1993) have examined this latter suggestion and derive an eddy diffusion coefficient that accurately reproduces the observed $\mathrm{C}_{2} \mathrm{H}_{6} / \mathrm{C}_{2} \mathrm{H}_{6}$ ratio using a photochemical scheme similar to that adopted here. Although Romani et al. (1993) derive reasonable $\mathrm{C}_{2} \mathrm{H}_{6}$ and $\mathrm{C}_{2} \mathrm{H}_{2}$ abundances for the lower stratosphere, their derived abundances for $\mathrm{CH}_{4}$ and $\mathrm{C}_{2} \mathrm{H}_{6}$ do not compare well with the higher altitude UVS data.

In fact, the information at high altitudes, which arises mainly from the analysis of the Voyager UVS data (Yelle et al. 1993), is very difficult to reconcile with the lower altitude information, which arises mainly from groundbased and Voyager infrared observations (Kostiuk et al. 1992, Orton et al. 1992, Bézard et al. 1991). The ethane mixing ratio appears to be dropping rapidly with altitude in the Yelle et al. UVS analysis, yet all the infrared observations indicate that the ethane mixing ratio is still quite large at lower altitudes. Although it is theoretically possible that a judicious choice of the eddy diffusion coefficient could reproduce the observations to within the quoted uncertainties, it is also possible that very real latitudinal differences exist. The ground-based infrared observations (Kostiuk et al. 1992, Orton et al. 1992) are global averages and the Voyager IRIS data (Kostiuk et al. 1992, Bézard et al. 1991) used for the comparisons with our photochemical models are also averaged over a full Neptune disk. The UVS occultations occurred at two specific latitudes that may not be representative of the global average. In addition, the UVS data show some differences in derived abundances at the two occultation sites (Yelle et al. 1993) and the IRIS data show spatial variations in the $\mathrm{C}_{2} \mathrm{H}_{2}$ emission (Bézard et al. 1991), further suggesting that some latitudinal differences exist. It therefore may be unreasonable to attempt to simultaneously fit both sets of data. A related problem with model/data comparisons is that unresolved uncertainties exist in the data analyses. For instance, the strength of the observed infrared emission lines depends on the stratospheric temperatures as well as the abundance of the molecule causing the emission; as already discussed, temperatures in Neptune's stratosphere are not well known and may be latitudinally variable. The UVS lightcurves provide good information concerning relative altitudes, but the absolute altitude scale is less certain.

At this time, we cannot establish the reasons for the problems with the model/data comparisons, and we will not attempt to resolve the issue in this paper. Instead, we will examine two cases. In the first case, we consider that the altitude dependence of the UVS egress occultation results at $49^{\circ} \mathrm{S}$ latitude is representative of $30^{\circ} \mathrm{S}$ latitude and assume that the problems with the model/data comparisons at high altitude are due to the adopted photochemical scheme so that standard diffusion coefficient profiles are appropriate; we then use a chemistry/diffusion scheme that fits the high-altitude $\mathrm{CH}_{4}$ and $\mathrm{C}_{2} \mathrm{H}_{6}$ observations fairly well but that overestimates the $\mathrm{C}_{2} \mathrm{H}_{2}$ abundance at all altitudes and underestimates the $\mathrm{C}_{2} \mathrm{H}_{6}$ abundance at lower altitudes. Because we overpredict the acetylene abundance in this case, we may also overpredict the $\mathrm{C}_{2} \mathrm{H}_{6}$ downward flux and aerosol production rate by a factor of $\sim 2$, and we can take this fact into account during our predictions of the aerosol production rates. In the second case, we assume that the globalaverage infrared observations at lower altitudes are representative of $30^{\circ} \mathrm{S}$ latitude, that the photochemical scheme is accurate, and that the reason that photochemical models tend to underestimate the observed $\mathrm{C}_{2} \mathrm{H}_{6} /$ $\mathrm{C}_{2} \mathrm{H}_{2}$ ratio in the lower stratosphere is that standard eddy diffusion profiles are not appropriate; we then use a diffusion profile identical to that of Case $C$ in Romani et al. (1993) to estimate the aerosol production rates. In this case, the model reproduces the lower altitude data and the ethane/acetylene ratio but does not compare well with the Yelle et al. UVS high-altitude data. 


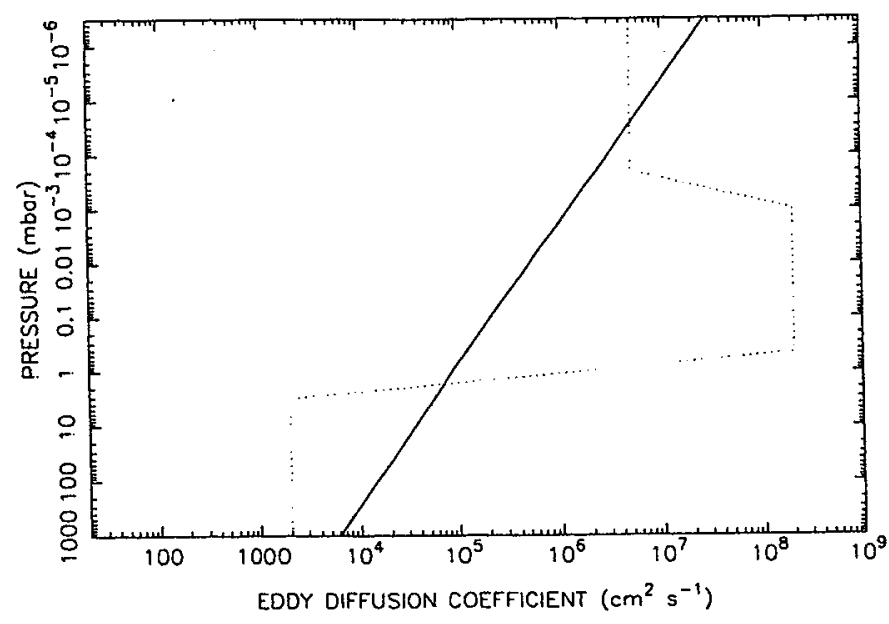

FIG. 4. The eddy diffusion coefficient used in our photochemical and aerosol-dynamics models. The solid line is our standard eddy diffusion profile and the dotted line is identical to Case C of Romani et al. (1993).

The actual eddy diffusion profiles used in our models are shown in Fig. 4. The solid line represents our standard model and is based on a modification of the traditional form for the eddy diffusion coefficient in a stably stratified atmosphere (Lindzen 1971):

$$
K=K_{1}\left(\frac{p_{1}}{p}\right)^{\beta}
$$

where $K_{1}$ and $p_{1}$ are the eddy coefficient and pressure at a reference level (e.g., the tropopause), and $\beta$ is close to the theoretically predicted value of 0.5 . We use this traditional form with $\beta=0.39, p_{1}=1 \mathrm{bar}$, and $K_{1}=6 \times 10^{3}$ $\mathrm{cm}^{2} \mathrm{sec}^{-1}$. These values for $\beta$ and $K_{1}$ were chosen so that the high-altitude UVS methane observations (Yelle et al. 1993) were reproduced given our choice of the methane mixing ratio in the lower stratosphere (see discussion below). The dotted profile in Fig. 4 is derived from Case $\mathrm{C}$ of Romani et al. 1993, and has $K=2 \times 10^{3} \mathrm{~cm}^{2} \mathrm{sec}^{-1}$ at pressures greater than $3 \mathrm{mbar}$, rapid growth in $K$ with decreasing pressure to $K=2 \times 10^{8} \mathrm{~cm}^{2} \mathrm{sec}^{-1}$ at $0.5 \mathrm{mbar}$, $K$ constant at $2 \times 10^{8} \mathrm{~cm}^{2} \mathrm{sec}^{-1}$ between 0.5 and $10^{-3}$ mbar, and $K$ falling again to $5 \times 10^{6}$ at pressures less than $2 \times 10^{-4}$ mbar.

The $\mathrm{H}_{2}$ density is held fixed in the photochemical model while the abundances of 38 hydrocarbon species are solved using the one-dimensional continuity equation that includes both photochemistry and molecular and eddy diffusion. Zero flux is assumed as an upper boundary condition for all the species except atomic $\mathrm{H}$, which is produced by EUV and soft-electron dissociation of $\mathrm{H}_{2}$ and is given a downward flux at the upper boundary of $4 \times 10^{7} \mathrm{~cm}^{-2} \mathrm{sec}^{-1}$ (Romani and Atreya 1988). The vol- ume mixing ratios of $\mathrm{He}(19 \%$, see Conrath et al. 1991b) and $\mathrm{CH}_{4}$ (either $7.5 \times 10^{-4}$ or $1 \times 10^{-4}$, depending on the model) are fixed at the lower boundary, and all other species are assumed to have a zero concentration gradient at the lower boundary (i.e., they are diffusing into the lower atmosphere at the maximum possible rate). Steady-state solutions to the diurnally averaged model are presented in Figs. 5 and 6.

Estimates concerning the methane mixing ratio $f_{\mathrm{CH}_{4}}$ in Neptune's lower stratosphere range from $\sim 10^{-4}$ to $10^{-3}$ (Bishop et al. 1992, Baines and Hammel 1994, Orton et al. 1992, Yelle et al. 1993). At this time, we cannot unambiguously determine the value of $f_{\mathrm{CH}_{4}}$ on Neptune because of uncertainties in stratospheric temperatures. We constrain $f_{\mathrm{CH}_{4}}$ in our standard model to be $7.5 \times 10^{-4}$ in the lower stratosphere based on the infrared observations of Orton et al. (1992) and on our assumptions concerning the stratospheric temperature profile. With this constraint in mind, the slope and magnitude of the eddy diffusion coefficient in the 0.1 - to $10^{-4}$-mbar region are adjusted until the standard model gives a reasonable fit to the observed $\mathrm{CH}_{4}$ and $\mathrm{C}_{2} \mathrm{H}_{6}$ concentrations from the Yelle et al. (1993) analysis of the UVS observations (see their Fig. 8f). We then assume that the eddy profile has the same slope down to at least the tropopause (116 mbar), and no further adjustments to the diffusion coefficient are made based on comparisons between the observations and model. Specifically, we have made no attempt to fit the other observations (e.g., $\mathrm{C}_{2} \mathrm{H}_{2}$ or $\mathrm{C}_{2} \mathrm{H}_{4}$ ) by adjusting the eddy diffusion profile because uncertainties are inherent in our photochemical scheme. Note that a different choice of $f_{\mathrm{CH}_{4}}$ would require us to choose a different slope $\beta$ and magnitude of $K$ in the $10^{-4}$-mbar region to reproduce the UVS observations. We cannot separate the effects of $K$ from $f_{\mathrm{CH}_{4}}$ in the data; thus, both values remain uncertain.

Figure 5 illustrates how our photochemical model compares with actual observations. The resulting ethane abundance in our standard model fits the Yelle et al. $\mathrm{C}_{2} \mathrm{H}_{6}$ observations reasonably well but is quite a bit smaller than the infrared $\mathrm{C}_{2} \mathrm{H}_{6}$ observations (Orton et al. 1992, Kostiuk et al. 1992, Bézard et al. 1991). The $\mathrm{C}_{2} \mathrm{H}_{2}$ abundance in the standard model is higher than all the observations, demonstrating the infamous problem with the $\mathrm{C}_{2} \mathrm{H}_{6} / \mathrm{C}_{2} \mathrm{H}_{2}$ ratio.

The results using the Case $\mathrm{C}$ eddy profile of Romani et al. (1993) appear much different. Romani et al. assume a $1 \times 10^{-4}$ methane mixing ratio in the lower stratosphere, based on models $a$ and $b$ of the Bishop et al. (1992) analysis of the UVS occultation data. By using the Romani et al. Case $C$ diffusion coefficient profile, most of the major hydrocarbon species are well mixed in the middle stratosphere, allowing the mixing ratios of these species to be constant with altitude. Although the Yelle et al. 

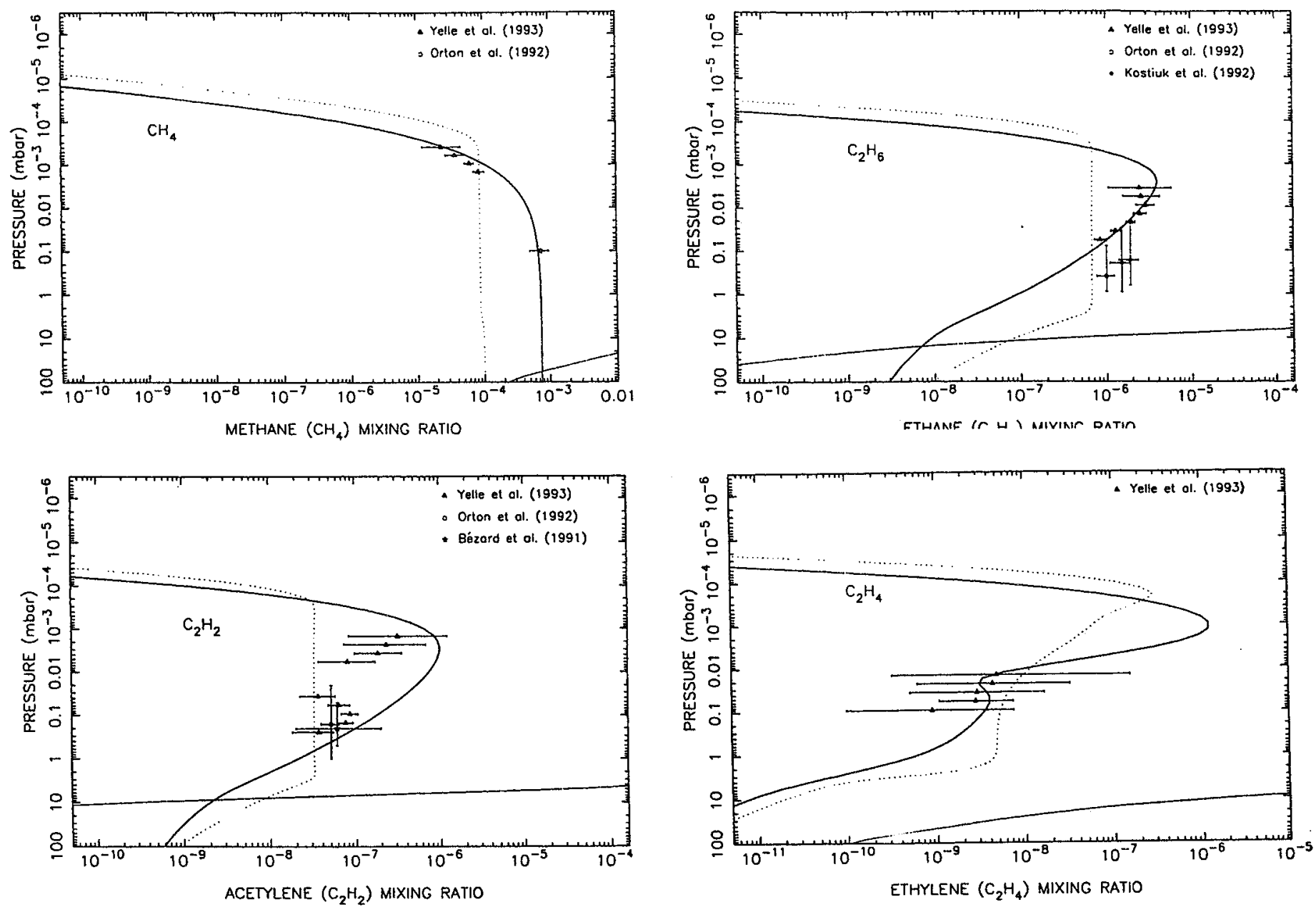

FIG. 5. The $49^{\circ} \mathrm{S}$ latitude results for several hydrocarbon species compared with various observations: (a) $\mathrm{CH}_{4}$, (b) $\mathrm{C}_{2} \mathrm{H}_{6}$, (c) $\mathrm{C}_{2} \mathrm{H}_{2}$, and (d) $\mathrm{C}_{2} \mathrm{H}_{4}$. The thick solid line represents the mixing ratios of the species in our standard model while the dotted line represents the results using the eddy profile from Case C of Romani et al. (1993) (see Fig. 4). The thinner solid lines near the bottom of the figures show the saturation vapor density profiles for each species. If the model abundances exceed the saturation vapor densities, then the species become supersaturated and might condense in this region. The triangle data points with associated error bars are from the UVS egress occultation results (Fig. $8 \mathrm{f}$ of Yelle et al. 1993), the open circles are from global-average ground-based infrared observations of Orton et al. (1992) assuming a stratospheric temperature of $168 \mathrm{~K}$, the solid circles are from the Voyager and ground-based infrared observations of Kostiuk et al. (1992), and the stars from an analysis of the Voyager IRIS data by Bezard et al. (1991). The horizontal error bars were taken directly from the above citations while the vertical error bars were determined from the contribution functions published in the above citations or sent via personal communication (e.g., G. Orton, 1992). No vertical error bars were assigned to the Yelle $e t$ al. data since we found the vertical errors difficult to interpret; however, these estimated errors should be nonzero. No vertical error bars were assigned to the Orton et al. $\mathrm{CH}_{4}$ data point due to lack of information; the contribution function will be broad and might not be centered at the pressure region at which we have assigned the point.

(1993) UVS analysis does not generally support constant mixing ratio profiles for most of the species, the Romani et al. model has the advantage of having a $\mathrm{C}_{2} \mathrm{H}_{6} / \mathrm{C}_{2} \mathrm{H}_{2}$ ratio that is close to observations. The mixing ratios derived for $\mathrm{C}_{2} \mathrm{H}_{6}$ and $\mathrm{C}_{2} \mathrm{H}_{2}$ in our Case $\mathrm{C}$ model differ a bit from those quoted by Romani et al. (1993) due to slight differences in our photochemical schemes; the implied downward flux of $\mathrm{C}_{2} \mathrm{H}_{2}$ at 6.9 mbar in our model $(2.7 \times$ $\left.10^{-16} \mathrm{~g} \mathrm{~cm}^{-2} \mathrm{sec}^{-1}\right)$ compares well with the Romani et al. quote from their Table VI $\left(2.8 \times 10^{-16} \mathrm{~g} \mathrm{~cm}^{-2} \mathrm{sec}^{-1}\right)$, but the $\mathrm{C}_{2} \mathrm{H}_{6}$ flux at $10 \mathrm{mbar}$ in our model $\left(5.1 \times 10^{-15} \mathrm{~g} \mathrm{~cm}^{-2}\right.$ $\mathrm{sec}^{-1}$ ) is $45 \%$ lower than the corresponding value from Table VI of Romani et al. $\left(7.4 \times 10^{-15}\right)$. We use the results from our model in later calculations.

At least eight of the methane photochemical products that we examine become supersaturated in Neptune's lower stratosphere. In order of decreasing importance to the possible aerosol production rate in our standard model, these species are ethane $\left(\mathrm{C}_{2} \mathrm{H}_{6}\right)$, acetylene $\left(\mathrm{C}_{2} \mathrm{H}_{2}\right)$, propane $\left(\mathrm{C}_{3} \mathrm{H}_{8}\right)$, diacetylene $\left(\mathrm{C}_{4} \mathrm{H}_{2}\right)$, methylacetylene 


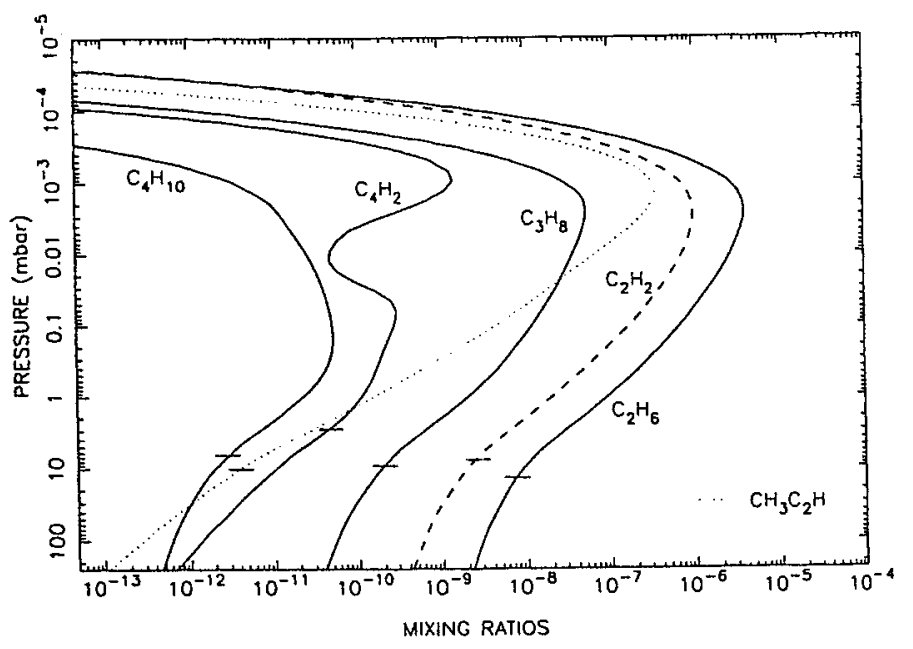

FIG. 6. The (volume) mixing ratios of the major condensible species in our standard photochemical model (for $30^{\circ} \mathrm{S}$ latitude). The small horizontal lines indicate the pressure levels at which the species become supersaturated.

$\left(\mathrm{C}_{3} \mathrm{H}_{4}\right.$, specifically $\left.\mathrm{CH}_{3} \mathrm{C}_{2} \mathrm{H}\right)$, butane $\left(\mathrm{C}_{4} \mathrm{H}_{10}\right)$, ethylacetylene $\left(\mathrm{C}_{4} \mathrm{H}_{6}\right)$, and propylene $\left(\mathrm{C}_{3} \mathrm{H}_{6}\right) . \mathrm{HCN}$, allene $\left(\mathrm{C}_{3} \mathrm{H}_{4}\right.$, specifically $\left.\mathrm{CH}_{2} \mathrm{CCH}_{2}\right)$, triacetylene $\left(\mathrm{C}_{6} \mathrm{H}_{2}\right)$, and higher order polyacetylenes may also contribute minor amounts to the total haze production rate. The concentrations of the most important condensible species in our standard model are shown in Fig. 6; the levels at which these species become saturated are also indicated in the figure. Remember that the photochemical model does not specifically include condensation; thus, the concentrations of the gas species below the saturation levels are not accurate-the actual concentrations will begin to mimic the saturation vapor density curves below these levels. Since the lower-stratospheric methane abundance adopted in our models requires that methane is supersaturated in Neptune's stratosphere, methane might also condense in Neptune's lower stratosphere (Fig. 7). In fact, methane could conceivably dominate the stratospheric haze production on Neptune.

TABLE II

Predictions from Standard Photochemical Model

\begin{tabular}{lcccc}
\hline Species & $\begin{array}{c}P_{\text {sat }} \\
\text { (mbar) }\end{array}$ & $\begin{array}{c}T_{\text {sat }} \\
(\mathrm{K})\end{array}$ & $\begin{array}{c}\text { Haze production rate } \\
\left(\mathrm{g} \mathrm{cm}^{-2} \mathrm{sec}^{-1}\right)\end{array}$ & $\begin{array}{c}\text { Fraction of } \\
\text { total }\end{array}$ \\
\hline $\mathrm{C}_{2} \mathrm{H}_{6}$ & 16 & 63 & $5.4 \times 10^{-15}$ & 0.814 \\
$\mathrm{C}_{2} \mathrm{H}_{2}$ & 9.4 & 72 & $1.0 \times 10^{-15}$ & 0.156 \\
$\mathrm{C}_{3} \mathrm{H}_{8}$ & 11 & 69 & $1.7 \times 10^{-16}$ & 0.026 \\
$\mathrm{C}_{4} \mathrm{H}_{2}$ & 3.4 & 106 & $1.4 \times 10^{-17}$ & 0.002 \\
$\mathrm{CH}_{3} \mathrm{C}_{2} \mathrm{H}$ & 12 & 67 & $5.3 \times 10^{-18}$ & 0.001 \\
$\mathrm{C}_{4} \mathrm{H}_{10}$ & 7.9 & 76 & $2.4 \times 10^{-18}$ & 0.000 \\
$\mathrm{C}_{4} \mathrm{H}_{6}$ & 9.4 & 72 & $6.3 \times 10^{-19}$ & 0.000 \\
$\mathrm{C}_{3} \mathrm{H}_{6}$ & 34 & 59 & $4.0 \times 10^{-19}$ & 0.000 \\
\hline
\end{tabular}

TABLE III

Predictions from Case C Photochemical Model

\begin{tabular}{lcccc}
\hline Species & $\begin{array}{c}P_{\text {sat }} \\
(\mathrm{mbar})\end{array}$ & $\begin{array}{c}T_{\text {sat }} \\
(\mathrm{K})\end{array}$ & $\begin{array}{c}\text { Haze production rate } \\
\left(\mathrm{g} \mathrm{cm}^{-2} \mathrm{sec}^{-1}\right)\end{array}$ & $\begin{array}{c}\text { Fraction of } \\
\text { total }\end{array}$ \\
\hline $\mathrm{C}_{2} \mathrm{H}_{6}$ & 12 & 67 & $5.1 \times 10^{-15}$ & 0.834 \\
$\mathrm{C}_{3} \mathrm{H}_{8}$ & 7.9 & 76 & $7.1 \times 10^{-16}$ & 0.115 \\
$\mathrm{C}_{2} \mathrm{H}_{2}$ & 7.9 & 76 & $2.8 \times 10^{-16}$ & 0.046 \\
$\mathrm{C}_{4} \mathrm{H}_{30}$ & 6.2 & 83 & $2.5 \times 10^{-17}$ & 0.004 \\
$\mathrm{C}_{4} \mathrm{H}_{2}$ & 3.2 & 108 & $5.5 \times 10^{-18}$ & 0.001 \\
$\mathrm{CH}_{3} \mathrm{C}_{2} \mathrm{H}$ & 10 & 70 & $1.5 \times 10^{-18}$ & 0.000 \\
$\mathrm{C}_{3} \mathrm{H}_{6}$ & 18 & 63 & $7.4 \times 10^{-19}$ & 0.000 \\
$\mathrm{C}_{4} \mathrm{H}_{6}$ & 8.6 & 74 & $1.2 \times 10^{-19}$ & 0.000 \\
\hline
\end{tabular}

The haze production rates for the individual species can be estimated based on the downward fluxes calculated from the photochemical model. The haze production rate predictions for our standard model and for the Case $\mathrm{C}$ diffusion profile model are shown in Tables II and III, respectively. Ethane clearly dominates the total haze production rate of the methane photolysis products in the stratosphere. Since we have overestimated the $\mathrm{C}_{2} \mathrm{H}_{2}$ abundance in our standard photochemical model, the acetylene haze production rate quoted in Table II is probably an overestimate; even so, acetylene and propane should make up the remainder of the stratospheric aerosol production, with minor but potentially observable amounts of the other species. The total haze production

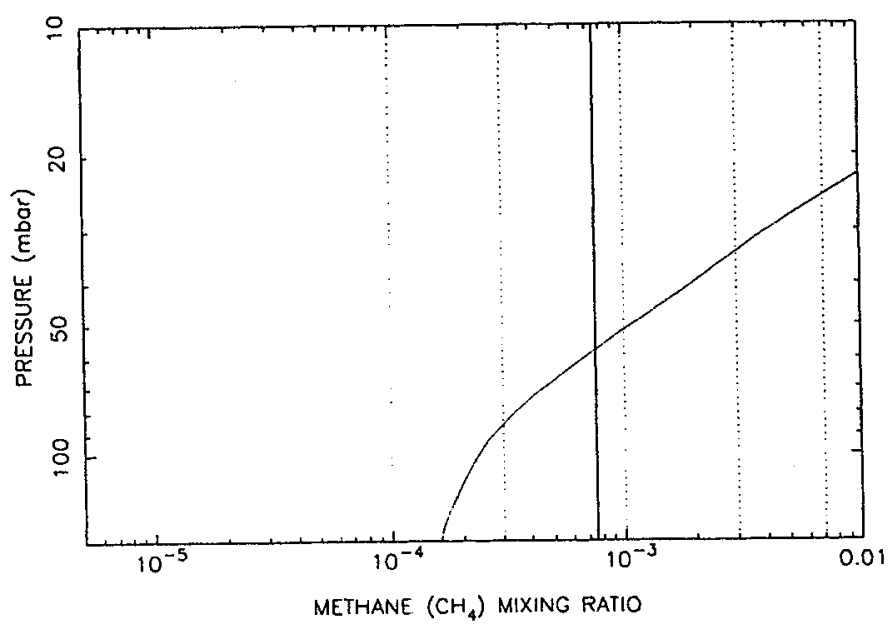

FIG. 7. The possible condensation levels for methane in Neptune's stratosphere as a function of methane mixing ratio. The solid line is the saturation vapor mixing ratio of methane for the temperature profile adopted in this model. The dotted vertical lines represent the nearly constant mixing ratio profiles that methane would have in the stratosphere in the absence of condensation. Methane can condense near the level where the actual mixing ratio exceeds the saturation vapor mixing ratio. Uncertainties in the temperature profile render these calculations approximate. 
rate from these eight species in the standard photochemical model is $6.6 \times 10^{-15} \mathrm{~g} \mathrm{~cm}^{-2} \mathrm{sec}^{-1}$ and from the Case $\mathrm{C}$ model is $6.1 \times 10^{-15} \mathrm{~g} \mathrm{~cm}^{-2} \mathrm{sec}^{-1}$.

Moses et al. (1992) demonstrate that the photolysis products may not condense right at their saturation levels because of the low vapor abundances of many of the species and because of difficulties with nucleation at the cold temperatures encountered in Neptune's stratosphere. In fact, if the hydrocarbon ice crystals that form at high altitudes due to condensation of some of the lower-volatility compounds do not provide congenial sites for the nucleation of embryos of the condensed phases of the more volatile species, then some of the species in Tables II and III might not nucleate at all. By calculating the heterogeneous nucleation rates in the manner discussed in Moses et al. (1992), we have attempted to predict the saturation ratios required for stable condensed-phase embryo formation of each of the hydrocarbon species onto any preexisting aerosol particles. These results are shown in Table IV. We have assumed here that the preexisting particles are large $(0.1 \mu \mathrm{m})$ based on some of the haze observations and are fairly compatible with the condensing phases (low contact angle, $5^{\circ}$ ).

Major species like ethane, acetylene, and propane are relatively unaffected by the finite nucleation rates while less abundant species like ethylacetylene never nucleate at an appreciable rate despite achieving relatively high supersaturations. Basically, ethylacetylene molecules rarely encounter each other on the surface of the aerosol particle, thus, a species like ethylacetylene cannot form a coherent condensed phase; however, that does not preclude the possibility that molecules or clusters of molecules of ethylacetylene and similar species might be in-

TABLE IV

Critical Levels Defining the Onset of Nucleation

\begin{tabular}{lcccc}
\hline Species & $P$ (mbar) & $T(\mathrm{~K})$ & $\begin{array}{c}\Delta z(\mathrm{~km}) \\
\text { from } S=1\end{array}$ & $S_{\text {crit }}$ \\
\hline $\mathrm{C}_{2} \mathrm{H}_{6}$ & 16 & 63 & 0 & 1.12 \\
$\mathrm{C}_{2} \mathrm{H}_{2}$ & 9.4 & 72 & 0 & 1.12 \\
$\mathrm{C}_{3} \mathrm{H}_{8}$ & 11 & 69 & 0 & 1.24 \\
$\mathrm{C}_{4} \mathrm{H}_{2}$ & 10 & 70 & -28 & $3 \times 10^{12}$ \\
$\mathrm{CH}_{3} \mathrm{C}_{2} \mathrm{H}$ & 15 & 65 & -4 & 5.76 \\
$\mathrm{C}_{4} \mathrm{H}_{10}$ & 16 & 63 & -16 & $5.9 \times 10^{3}$ \\
$\mathrm{C}_{3} \mathrm{H}_{6}$ & 38 & 58 & -2 & 1.67 \\
\hline
\end{tabular}

Note. The calculations are for heterogeneous nucleation about a spherical insoluble particle with radius $r_{N}=0.1 \mu \mathrm{m}$ and contact angle $\theta=5^{\circ}$ (see Moses et al. 1992 for a definition of these terms). The critical saturation ratio $S_{\text {crit }}$ is defined as the saturation ratio $S$ at which the nucleation rate reaches $10^{-2} \mathrm{~cm}^{-3} \mathrm{sec}^{-1}$. Diacetylene $\left(\mathrm{C}_{4} \mathrm{H}_{2}\right)$ does not nucleate effectively by heterogeneous nucleation but may by ion-induced nucleation (Moses et al. 1992). corporated into the matrix of other condensed phases. Since the major haze-forming species do not require large supersaturations to initiate nucleation, we use the information in Table II as our input for further calculations on the aerosol evolution. The biggest uncertainty in our prediction of the actual levels at which condensation will occur is not our uncertainties in the details of nucleation-it is the uncertainty in the temperature profile itself and in the photochemistry. Because of the exponential dependence of the vapor pressures on temperature, small changes in temperature can lead to large changes in the predicted condensation levels. As mentioned before, we do not know the temperature profile accurately in the lower stratosphere at the latitudes covered by the highphase-angle Voyager images. Differences in the photochemical schemes and in the adopted diffusion profile can lead to large differences in the predicted abundances of the condensible species in the lower stratosphere (see Fig. 5), which can also affect the predicted condensation levels (cf. Tables II and III).

\subsection{Aerosol Dynamics}

We use an aerosol-dynamics model similar to that described in Pollack et al. (1987) to follow the formation and evolution of the stratospheric haze particles on Neptune. Our model is based on the one-dimensional physical-chemical aerosol models of Turco et al. (1979) and Toon et al. (1980), which were developed to simulate aerosol formation in the stratospheres of the Earth and Titan. In our Neptune model, we solve the one-dimensional aerosol continuity equation for the stratospheric aerosol particles using an implicit "backward Euler" technique. We let small particles form at high altitudes on Neptune due to processes such as the ablation and recondensation of meteoric debris (e.g., Moses 1992) or the condensation of relatively involatile disequilibrium photochemical products formed from either ultraviolet photolysis or charged-particle dissociation of methane and other atmospheric gases (e.g., Khare et al. 1987). These high-altitude particles then diffuse or settle into the lower stratosphere where they eventually reach the hydrocarbon condensation regions. Certain hydrocarbon species then condense about the particles, and the particles grow by vapor deposition and Brownian coagulation and move vertically due to gravitational settling and eddy diffusion. The model conserves mass, does not introduce appreciable artificial numerical dispersion, and has been tested against simple analytical solutions as described in Pollack et al. (1987).

Using the hydrostatic model atmosphere described in Appendix B, we divide Neptune's atmosphere into 127 discrete altitude bins with each bin separated by $2 \mathrm{~km}$ in the lower and middle stratosphere and by less than $1 / 3$ of 
a scale height in the upper stratosphere. All aerosol particles in the model are assumed to be spherical and are segregated into size bins that double in volume with each successive bin. Particles are formed in the smallest size bin only at the high altitudes; in the lower altitude hydrocarbon-condensation regions, no new particles are formed, but vapor condenses directly onto the preexisting particles. Because the data indicate that the preexisting particles are fairly large even at high altitudes on Neptune, nucleation will probably be rapid for the major haze-forming gases (see Section 3.2); thus, nucleation is not considered explicitly. In addition, because the particles do not grow large enough to have coalescence become important, this process is neglected. Zero flux is assumed as a boundary condition at the top of the model $\left(10^{-3} \mathrm{mbar}\right)$, and the particle velocity at the lower boundary is defined by the sedimentation velocity in the lowest layer and by the fastest possible eddy diffusion velocity (the diffusion coefficient divided by the scale height) in the lowest layer (1 bar). The model is allowed to evolve to steady state. It generally takes $\sim 800$ model years until the mass input near the top of the model is balanced by the mass flowing out the bottom.

We use the silicate meteoroid ablation profiles of Moses (1992) to estimate the particle production rate in Neptune's upper atmosphere and assume that the ablated debris immediately recondenses to form particles in the smallest size bin in our model. The condensation flux and initial size of these newly created particles are essentially free parameters in the model. Moses suggests ablation fluxes in the range of $2 \times 10^{-17}$ to $4 \times 10^{-15} \mathrm{~g} \mathrm{~cm}^{-2} \mathrm{sec}^{-1}$ and initial sizes of 0.001 to $0.01 \mu \mathrm{m}$ for these particles. We use the smallest flux and the largest particle size in these ranges for our initial guess.

Several of the other free parameters in the aerosol model can be constrained from previous information. For instance, the relative haze production rates (condensation fluxes) and the location of the hydrocarbon condensation levels can be obtained from the photochemical models described in Section 3.2; we use the information in Table II for our initial guess. The particle bulk density can also be considered a free parameter and will affect the results; we assume that the hazes have an altitudeindependent bulk density that is determined by the massflux-weighted average of the "silicate" debris (with assumed density of 1.5 to $3 \mathrm{~g} \mathrm{~cm}^{-3}$ ) and the hydrocarbon ices, represented by ethane (with a density of $0.7 \mathrm{~g} \mathrm{~cm}^{-3}$ ). Other free parameters in the model include the average charge on the particles (i.e., coagulation is inhibited if particles are similarly charged), the eddy diffusion coeffcient in the condensation region, and the background atmospheric density and temperature variation with altitude. We use the hydrostatic and photochemical models discussed in Sections 3.1 and 3.2 to develop an initial guess for the latter two parameters. Comparisons between models and observations help us further constrain these free parameters.

\subsection{Rayleigh and Mie Scattering}

In the final step of our forward modeling of Neptune's stratospheric hazes, we use the results from the aerosoldynamics model to predict the amount of light scattered from the haze particles in Neptune's stratosphere. A Mie-scattering routine developed originally by Toon and Ackerman (1981) to derive the aerosol single-scattering albedo, phase function, and extinction coefficient as a function of altitude is used, and then these results are combined with standard single-scattering Rayleigh calculations to predict the total extinction per unit path length in the stratosphere. Afterward, the extinction coefficient profiles are compared directly with observations.

Much of what we observe in the high-phase-angle Voyager images is extinction due to Rayleigh scattering from gas molecules in Neptune's atmosphere. To estimate the magnitude of this effect, we first divide the atmosphere into homogeneous layers $2 \mathrm{~km}$ thick and assume that the atmosphere is composed of $19 \%$ helium, 0.075 or $0.01 \%$ methane (depending on the model), with the remainder being hydrogen (see Appendix B for more details about the background atmosphere). Note that the methane homopause lies above the region in which the ISS observations are sensitive; thus, the methane mixing ratio has not started to drop notably, and we can assume that it is constant with altitude. Methane condensation in the lower stratosphere would have a negligible effect on the results. In the Voyager continuum filters, absorption by gaseous methane, hydrogen, and helium in Neptune's stratosphere is negligible; therefore, the imaginary index of refraction of these gas molecules is assumed to be zero. The Rayleigh extinction coefficient $\beta_{\text {Ray }}$ can then be calculated for a series of monochromatic wavelengths, and these values convolved over the Voyager camera/ filter spectral response functions. According to van de Hulst (1957),

$$
\beta_{\text {Ray }}(\lambda)=\frac{32 \pi^{3}\left(n_{\mathrm{r}}(\lambda)-1\right)^{2}}{3 n \lambda^{4}} f
$$

where $n_{\mathrm{r}}$ is the real refractive index of the gas, $\lambda$ is the wavelength, $n$ is the concentration of gas molecules $\left(\mathrm{cm}^{-3}\right)$, and $f$ is a constant of order unity that depends on the depolarization factors discussed in van de Hulst (1957). Filter response profiles are given in Danielson (1981); more recent recalibrations of these response functions (T. V. Johnson, personal communication, 1988) have been used throughout this paper. The Rayleigh phase function $p(\theta)_{\text {Ray }}$ equals $0.75\left(1+\cos ^{2} \theta\right)$, where $\theta$ is 
the angle between the incident and scattered light. Note that $\theta$ equals $180^{\circ}$ minus the phase angle of the observations.

The contribution to the total observed extinction due to scattering from haze particles becomes progressively more important at lower altitudes in Neptune's stratosphere. The monochromatic aerosol extinction coefficient $\beta_{\text {haz }}(\lambda)$ is calculated by integrating the extinction cross section of the particles over the particle size distribution; i.e.,

$$
\beta_{\mathrm{haz}}(\lambda)=\int_{0}^{\infty} Q_{\mathrm{ext}}(\lambda, r) \pi r^{2} n(r) d r,
$$

where $r$ is the particle radius, $n(r)$ is the particle size distribution calculated in Section 3.3, and $Q_{\text {ext }}(\lambda, r)$ is the Mie-scattering extinction efficiency of the particles calculated using a routine similar to that of Toon and Ackerman (1981). In Neptune's stratosphere, ethane and other hydrocarbon ices will condense about preexisting aerosol particles composed of silicate meteoric debris, high-volatility hydrocarbons, or other ices. To simulate this effect, the particles in the Mie-scattering routine are assumed to consist of a spherical core of one material surrounded by a spherical mantle or shell of another material. The optical properties of certain material classes that may be relevant to the situation in Neptune's stratosphere are listed in Table V. Here, $n_{\mathrm{r}}$ and $n_{\mathrm{i}}$ are the real and imaginary refractive indices, $\lambda$ is the wavelength in micrometers, and the values are representative of the material types but should not be considered exact (most values are obtained from standard physics and chemistry handbooks, Rizk et al. 1991, or Pollack et al. 1987). The imaginary index of refraction for the material labeled "kerogen" is actually taken from the derived refractive index of the Uranus stratospheric hazes (Rages et al. 1991), and pure ethane has a imaginary refractive index smaller than that quoted, but it is unlikely that pure ethane ice as such exists in Neptune's atmosphere. The aerosol single-scattering albedo $\bar{\omega}_{0}$ and phase function $p(\theta)$ are also calculated using the Mie-scattering routine. All calculations are performed for a series of monochromatic wavelengths, and the results are convolved over the Voyager camera/filter spectral response functions.

The total extinction coefficient and albedo-weighted phase function due to Rayleigh and aerosol scattering in any altitude layer can be calculated by

$$
\beta_{\mathrm{tot}}=\beta_{\mathrm{haz}}+\beta_{\mathrm{Ray}}
$$

and

$$
\left(\tilde{\omega}_{0} p(\theta)\right)_{\mathrm{tot}}=\frac{\beta_{\mathrm{haz}}\left(\tilde{\omega}_{0} p(\theta)\right)_{\mathrm{haz}}+\beta_{\mathrm{Ray}} p(\theta)_{\mathrm{Ray}}}{\beta_{\mathrm{tot}}},
$$

TABLE V Optical Constants

\begin{tabular}{llc}
\hline Material & $n_{\mathrm{r}}$ & $n_{\mathrm{i}}$ \\
\hline Rock & 1.7 & $3 \times 10^{-4}$ \\
Kerogen & 1.68 & $\exp (-2.1-6.5 \cdot \lambda)$ \\
Ethane & 1.44 & $1 \times 10^{-4}$ \\
Methane & 1.3 & $1 \times 10^{-6}$ \\
\hline
\end{tabular}

where the subscripts Ray, haz, and tot refer to Rayleigh, haze, and total.

\section{RESULTS AND DISCUSSION}

We now present the results of our data inversion and theoretical simulations. The theoretical models discussed in Section 3 provide our first estimates as to the haze production rates, condensation levels, and other parameters required for the aerosol modeling; however, the aerosol distributions that result from these first guesses were found to poorly reproduce the data. Therefore, we were forced to alter our initial parameters, in some cases substantially, before we could obtain a good fit between models and data. In the following section, we discuss the haze parameters that best fit the data, discuss the sensitivity of the aerosol model to the various free parameters, and provide constraints on the major physical properties of interest in the stratospheric haze layers.

\subsection{Extinction Coefficient Profile}

Figure 8 shows the results of the data inversion for the NA camera clear-filter image taken near $29.4^{\circ} \mathrm{S}$ latitude at a $158^{\circ}$ solar phase angle. The thick solid line with associated error bounds represents the total observed "extinction" due to both molecular Rayleigh scattering and aerosol scattering and absorption. The figure is actually a plot of the product of the extinction coefficient $\beta$, singlescattering albedo $\tilde{\omega}_{0}$, and phase function $p(\theta)$ rather than a plot of just the extinction coefficient because we find it difficult to separate the effects of $\beta$ from those of $\tilde{\omega}_{0} p(\theta)$ in the data. The difficulty stems from that fact that the observed specific intensity $I / F$ is proportional to $\tilde{\omega}_{0} p(\theta)$ times the cumulative extinction coefficient (i.e., optical depth) at slant optical depths much less than 1 while it is proportional to $\tilde{\omega}_{0} p(\theta)$ alone as the atmosphere becomes optically thick; the expression is more complicated at intermediate optical depths (see Pollack et al. 1987). From the data inversion procedure discussed in Appen$\operatorname{dix} A$, we find that we can separate $\beta$ from $\tilde{\omega}_{0} p(\theta)$ only near and below the altitude marked by a "bullet" in the figure; i.e., at a region in which the slant path through the atmosphere becomes optically thick, but before signal 


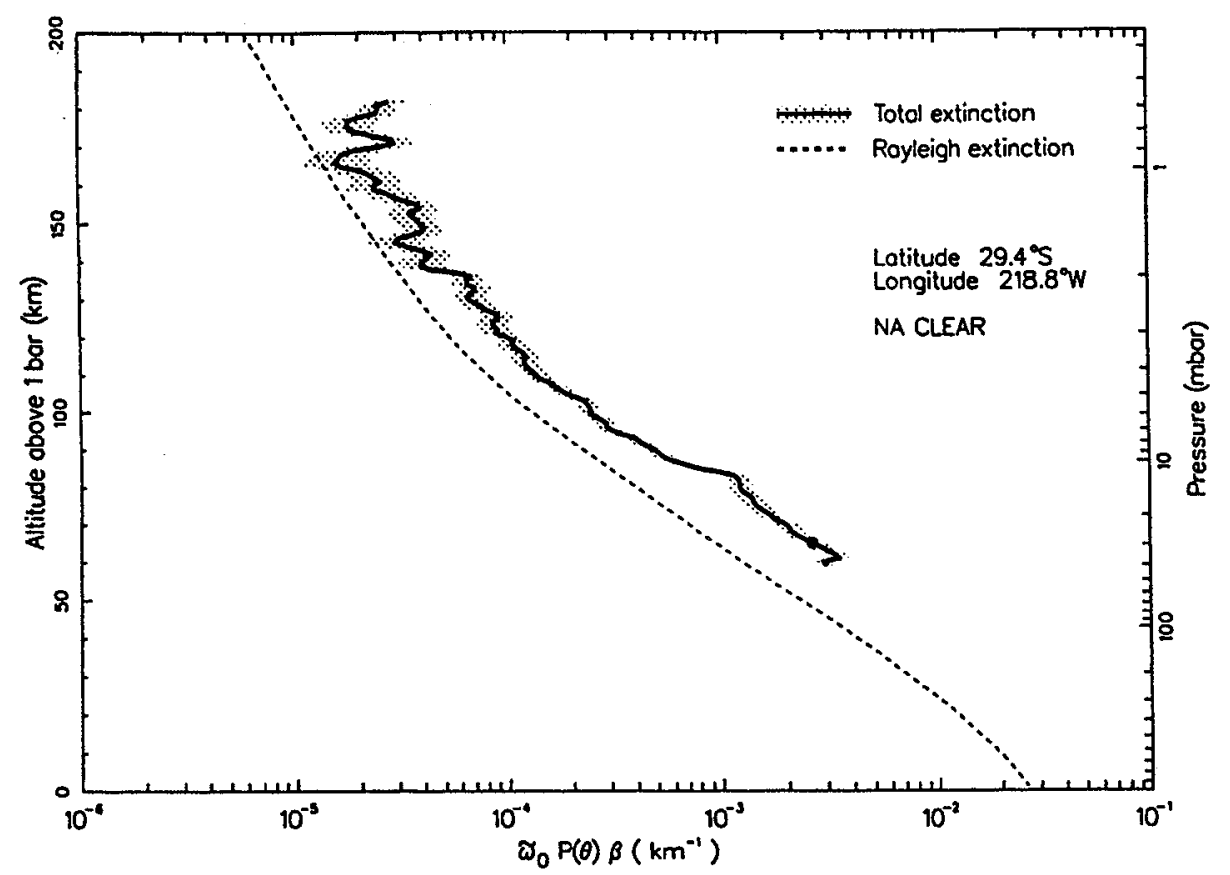

FIG. 8. Vertical profile of the extinction coefficient times the albedo-weighted phase function for the $158^{\circ}$ phase-angle clear-filter observations of Neptune's stratosphere. The thick solid line with shaded error bounds depicts the actual inverted data whereas the dashed line illustrates the predicted molecular Rayleigh extinction profile. Although the total observed extinction appears to exceed the Rayleigh curve at all altitudes in the stratosphere, the absolute altitude scale of the observations is uncertain. At the point marked with a bullet on the observation profile, the albedoweighted phase function $\bar{\omega}_{0} p(\theta)$ is inferred to be 2.52 , significantly above the Rayleigh phase function of 1.40 , implying the presence of haze particles at least in this altitude region.

quality is lost. Any reasonable aerosol model must reproduce both $\beta$ and $\bar{\omega}_{0} p(\theta)$ in this altitude region. The singlescattering albedo-weighted phase functions derived by our inversion procedure for the different filters in this bullet region are shown in Table VI along with the Rayleigh phase function calculated for an appropriate average phase angle for the images and other information relevant to the limb scans.

Two main results are obvious from Fig. 8. First of all, the extinction increases rapidly with altitude in the $\sim 12-$

TABLE VI

Limb Scan Characteristics

\begin{tabular}{lcccc}
\hline \multicolumn{1}{c}{ Filter } & $\lambda_{\text {enf }}{ }^{c}(\mu \mathrm{m})$ & $\tilde{\omega}_{0} p(\theta)_{\text {Ray }}{ }^{b}$ & $\dot{\omega}_{0} p(\theta)_{\mathrm{haz}^{c}}$ & $P_{\mathrm{b}}(\mathrm{mbar})^{d}$ \\
\hline NA clear & 0.473 & 1.40 & 2.52 & 28 \\
WA violet & 0.431 & 1.41 & 2.54 & 22 \\
WA blue & 0.474 & 1.41 & 2.45 & 31 \\
WA green & 0.562 & 1.41 & 2.85 & 58 \\
WA orange & 0.596 & 1.41 & 2.68 & 72 \\
\hline
\end{tabular}

a Effective wavelength for the Voyager filters.

b Differences are due to differences in the phase angle of the images.

- Model-independent determination.

¿ Pressure of the "bullet" region (where $\beta_{\mathrm{tot}}=10^{-3} \mathrm{~km}^{-1}$ for Model A). Note that $P_{\mathrm{b}}$ is a model-dependent quantity. mbar region, suggesting the presence of a distinct haze layer in Neptune's lower stratosphere. Secondly, the total observed extinction exceeds the Rayleigh extinction throughout the region of stratosphere probed by the observations, suggesting that hazes contribute measurably to the scattering at all altitudes in Neptune's stratosphere. The second result is somewhat surprising (cf. the Uranus results reported by Pollack et al. 1987 and Rages et al. 1991) and deserves more discussion since the conclusion also depends on the uncertain altitude scale of the observations.

All the limb scans at the different latitudes in the images taken with the different filters were converted to equivalent equatorial radii to facilitate comparisons between the different filters. The data in Fig. 8 are plotted as a function of equatorial altitude above an assumed 1bar equatorial radius of $24764 \mathrm{~km}$ (Lindal et al. 1990). Due to ambiguities in camera pointing and in the planetary shape, the absolute altitude scale of the observations is uncertain. For instance, errors in the camera pointing or geometry or the assumed planetary oblateness may cause the data in Fig. 8 to be shifted up or down relative to the model atmosphere by 10 s of kilometers (see Appendix A). If we are off by $10 \mathrm{~km}$, the observations could be shifted down in altitude such that the high-altitude 
data more closely follow the Rayleigh extinction curve. In that case, haze scattering would play a minor role at high altitudes. We will discuss this problem in more detail later.

When we compare the aerosol models with the data, the absolute altitude scale of the observations is derived in the following manner. We first determine the total extinction coefficient $\beta$ of the observations in the altitude region (marked by a bullet in Fig. 8 ) at which we think we can separate the effects of the extinction and the albedoweighted phase function. The total extinction coefficient in this region is $\sim 1 \times 10^{-3} \mathrm{~km}^{-1}$. We then determine the location at which the total extinction in the aerosol models reaches $1 \times 10^{-3} \mathrm{~km}^{-1}$ for each filter and line up the two altitude scales at that point. The resulting "absolute" altitude scale of the observations is model dependent. Luckily, the different Voyager filters sample different altitude regions; thus, we have several constraints on the models.

\subsection{Comparisons with Models}

Initial attempt. For our first modeling attempt, we use the results from the standard photochemical model to predict the haze condensation levels and the relative haze production rates for the different species (see Table II). We also use the ablation results of Moses (1992) and assume that small haze particles $(0.01 \mu \mathrm{m}$ radius) are produced at high altitudes (e.g., 0.1 to 0.001 mbar) at a rate of $2 \times 10^{-17} \mathrm{~g} \mathrm{~cm}^{-2} \mathrm{sec}^{-1}$. Based on the results for Uranus (Rages et al. 1991), we assume that the particles have an average charge of $10 e^{-}$per micrometer, and we require that the average material bulk density of the particles be 0.706 (e.g., a mass-flux-weighted average of the high-altitude involatile material density, $\sim 2.5 \mathrm{~g} \mathrm{~cm}^{-3}$, and the hydrocarbon ice density, $\sim 0.7 \mathrm{~g} \mathrm{~cm}^{-3}$ ).

The aerosol distribution resulting from this model has particles that remain small at high altitudes due to low coagulation rates but that grow rapidly in the hydrocarbon condensation region due to high condensation rates. At $1 \mathrm{mbar}$, the mean particle radius in this model is just $0.02 \mu \mathrm{m}$; however, the particles grow to $0.2 \mu \mathrm{m}$ by 20 mbar and to $0.3 \mu \mathrm{m}$ by $1 \mathrm{bar}$. To determine the scattering properties of the aerosols in the model, we assume that the particles are composed of a kerogen core surrounded by an ethane mantle (see Table V), with the altitude independent core/particle radius determined by the relative mass fluxes of the high-altitude involatile material (e.g., kerogen or silicate material) and the hydrocarbon ices, and we use the Rayleigh and Mie-scattering code described in Section 3.4 to determine the albedo-weighted phase function and extinction coefficient that would result from the calculated aerosol distribution. The results are shown in Figs. 9 and 10.
Figure 9 shows the actual phase function and extinction coefficient derived from the calculated aerosol distribution. The aerosol phase function remains low and Rayleigh scattering dominates the extinction profile while the particles are small, but both the phase function and extinction rise dramatically in the condensation region as the particles grow large. The observed total phase function at the bullet region in the clear-filter data is 2.52 while the total phase function of the model (calculated from Eq. (2)) is 4.70 at the same altitude level (where the absolute altitude scale of the observations is determined from $\beta_{\text {tot }}$ in the model-data comparison as discussed in Section 4.1). Since particle size has the largest effect on the phase function at these low particle concentrations, it seems that we are overestimating the particle size in the condensation regions in the model. Moreover, a comparison of the product of $\beta$ and $\bar{\omega}_{0} p(\theta)$ between the model and observations (Fig. 10) demonstrates that our initial model provides a very poor fit to the data at all altitude levelswe underestimate the total extinction at high altitudes and overestimate the extinction in the hydrocarbon condensation region.

To obtain a better fit, we would need to (1) increase the production rate of the involatile high-altitude hazes in the model and/or increase the particle size of the high-altitude hazes and (2) decrease the haze production rate in the lower stratosphere. In fact, subsequent aerosol modeling demonstrates that the current predictions from photochemical models grossly overestimate the effective aerosol production rate in Neptune's lower stratosphere.
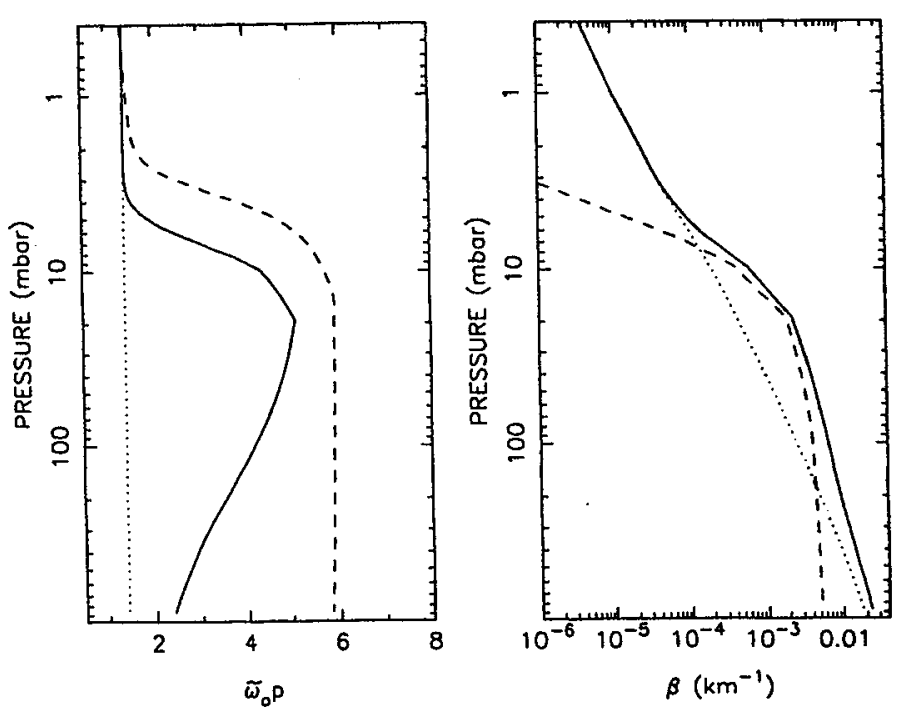

FIG. 9. The calculated total albedo-weighted phase function $\bar{\omega}_{0} p(\theta)$ and cumulative extinction coefficient $\beta$ in our first model. The dotted line represents the results for Rayleigh scattering only, the dashed line for aerosol extinction only, and the solid line for the combination of aerosol and Rayleigh extinction (see Eqs. (1) and (2)). 


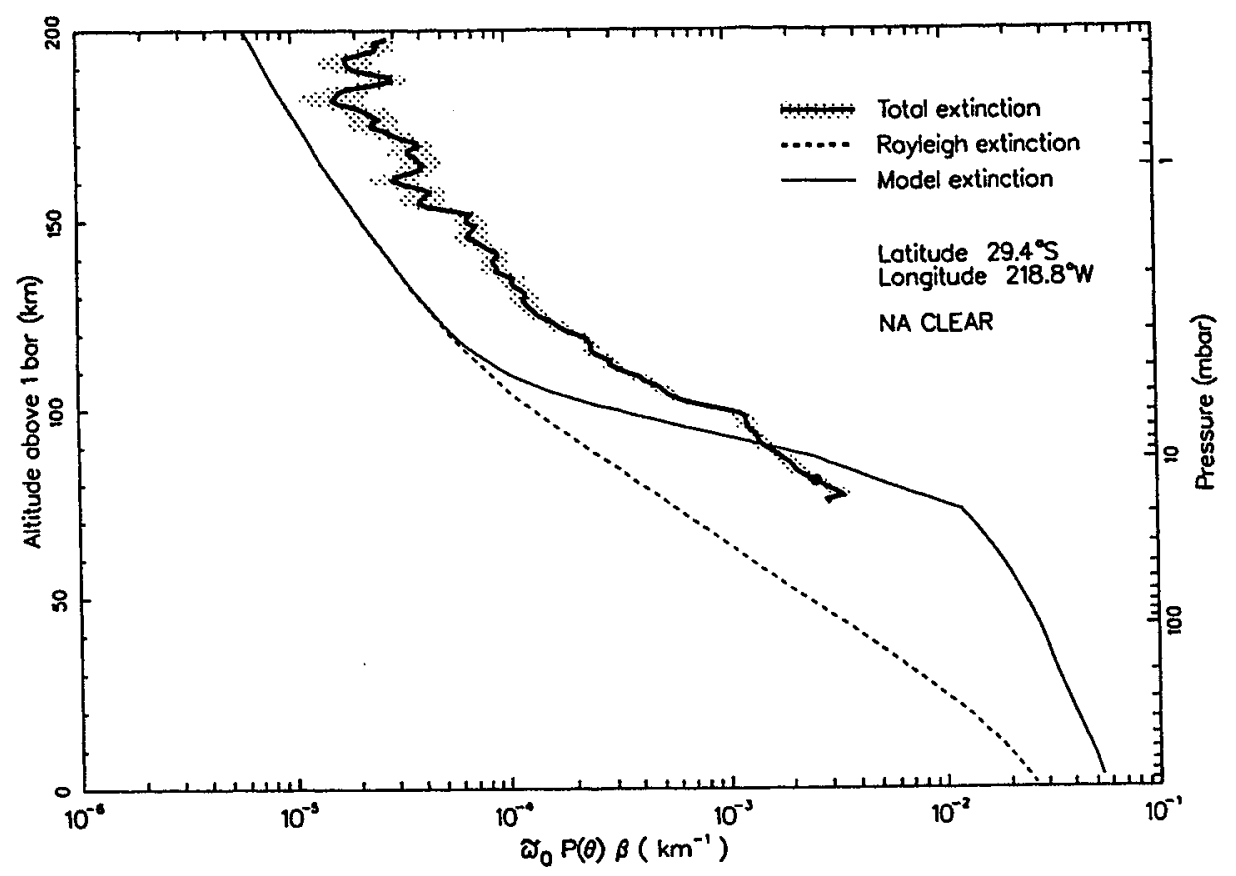

FIG. 10. A comparison between the clear-filter observations and our first-guess aerosol model. The model grossly overestimates the product of the extinction coefficient and the albedo-weighted phase function in Neptune's lower atmosphere.

Even by adjusting the other free parameters in the model, we are never able to develop an aerosol model that comes close to fitting the data when we use haze production rates similar to those quoted in Table II and III or in Romani et al. (1993).

Best-fit model (Model $A$ ). In an attempt to reproduce the observations, we varied the model free parameters in a somewhat systematic manner and created over 200 different aerosol models. Although we have not completely explored parameter space at this time, the model that fits the observations best is shown in Fig. 11 and Table VII. At high altitudes ( 0.1 to 0.001 mbar), the creation flux of small particles in the best-fit model (hereafter called Model A) is found to be $7 \times 10^{-16} \mathrm{~g} \mathrm{~cm}^{-2} \mathrm{sec}^{-1}$; the highaltitude particles are assumed to have an initial radius of $0.09 \mu \mathrm{m}$. The total haze production rate in the hydrocarbon condensation region is $1.0 \times 10^{-15} \mathrm{~g} \mathrm{~cm}^{-2} \mathrm{sec}^{-1}$, divided such that $4.0 \times 10^{-17} \mathrm{~g} \mathrm{~cm}^{-2} \mathrm{sec}^{-1}$ condenses at 5 mbar, $1.0 \times 10^{-16} \mathrm{~g} \mathrm{~cm}^{-2} \mathrm{sec}^{-1}$ condenses at $7.9 \mathrm{mbar}$, $8.0 \times 10^{-17} \mathrm{~g} \mathrm{~cm}^{-2} \mathrm{sec}^{-1}$ condenses at $8.6 \mathrm{mbar}, 8.0 \times$ $10^{-17} \mathrm{~g} \mathrm{~cm}^{-2} \mathrm{sec}^{-1}$ condenses at $10 \mathrm{mbar}$, and $7.0 \times 10^{-16}$ $\mathrm{g} \mathrm{cm}^{-2} \mathrm{sec}^{-1}$ condenses at $12 \mathrm{mbar}$. The different condensation levels were chosen somewhat arbitrarily, but might loosely correspond to the condensation of $\mathrm{HCN}$, $\mathrm{C}_{4} \mathrm{H}_{2}$, and $\mathrm{C}_{4} \mathrm{H}_{10}$ at the highest altitudes (lowest pressures), followed by the condensation of $\mathrm{C}_{2} \mathrm{H}_{2}$ and $\mathrm{C}_{3} \mathrm{H}_{8}$ at intermediate pressures, and ultimately the bulk of the ethane condensation occurring near 12 mbar. The aver- age charge on the particles is assumed to be $13 e^{-}$per micrometer, and the mean bulk density is calculated as before; i.e., by taking the mass-flux-weighted average of the assumed higher density $\left(2.8 \mathrm{~g} \mathrm{~cm}^{-3}\right)$ high-altitude particles and the lower density $\left(0.7 \mathrm{~g} \mathrm{~cm}^{-3}\right)$ hydrocarbon ices. The model fits the clear, blue, and green filter data well, but seems to have a slight systematic trend away from a good fit as we go to shorter or longer wavelengths.

The particle concentration and mean particle size in our Model A are shown in Figs. 12 and 13. The mean

TABLE VII

Sensitivity to Free Parameters

\begin{tabular}{|c|c|c|}
\hline Parameter & Base value & $\begin{array}{c}\text { Sensitivity to } \\
\text { variation }\end{array}$ \\
\hline Condensation flux & $1 \times 10^{-15} \mathrm{~g} \mathrm{~cm}^{-2} \mathrm{sec}^{-1}$ & High \\
\hline Creation flux & $7 \times 10^{-16} \mathrm{~g} \mathrm{~cm}^{-2} \mathrm{sec}^{-1}$ & High \\
\hline $\begin{array}{l}\text { Radius of newly created } \\
\text { particles }\end{array}$ & $0.09 \mu \mathrm{m}$ & High \\
\hline Material bulk density & $1.56 \mathrm{~g} \mathrm{~cm}^{-3}$ & High \\
\hline Eddy diffusion coefficient at & $3 \times 10^{4} \mathrm{~cm}^{2} \mathrm{sec}^{-1}$ & Moderate \\
\hline Charge & $13 e^{-1 / \mu \mathrm{m}}$ & Moderate \\
\hline $\begin{array}{l}\text { Stratospheric temperature } \\
\text { profile }\end{array}$ & & Moderate \\
\hline Condensation levels & Variable & Low \\
\hline Optical properties & Kerogen core, ethane mantle & Low \\
\hline
\end{tabular}




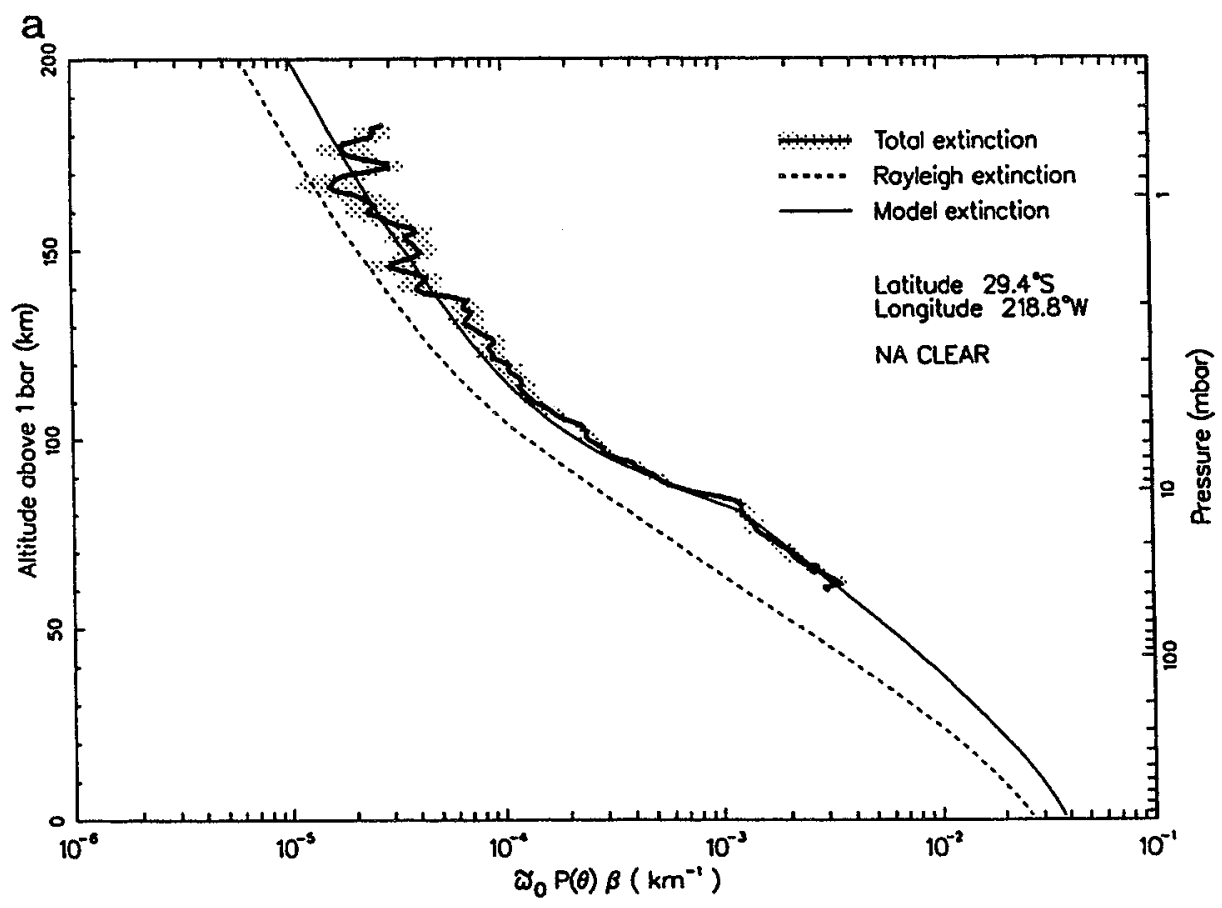

FIG. 11. A comparison of our best-fit model (Model A) with observations from the (a) clear, (b) violet, (c) blue, (d) green, and (e) orange Voyager filters. The NA clear-filter data have a higher spatial resolution than that of the WA colored filters; thus the distinct haze layer shows up more clearly in the clear-filter image. The shaded error bounds represent the formal error in the SVD inversion routine but do not take other systematic errors (e.g., the altitude uncertainty) into account.

particle size is plotted three ways: as the arithmetic mean radius of the particle distribution

$$
\langle r\rangle=\frac{\int_{0}^{\infty} n(r) r d r}{\int_{0}^{x} n(r) d r},
$$

where $r$ is the particle radius and $n(r)$ is the particle size distribution, as the effective or area-weighted mean radius

$$
\langle r\rangle_{\mathrm{aw}}=\frac{\int_{0}^{\infty} \pi r^{2} n(r) r d r}{\int_{0}^{\infty} \pi r^{2} n(r) d r},
$$

and as the cross-section-weighted mean radius (mean radius for extinction)

$$
\langle r\rangle_{\mathrm{csw}}=\frac{\int_{0}^{\infty} Q_{\mathrm{ext}}(r) \pi r^{2} n(r) r d r}{\int_{0}^{\infty} Q_{\mathrm{ext}}(r) \pi r^{2} n(r) d r},
$$

where $Q_{\text {ext }}(r)$ is the Mie extinction efficiency (van de Hulst 1957) calculated by convolution over the appropriate Voyager filter wavelengths. If the particle size distribution is broad, larger particles will contribute more to the observed atmospheric extinction; thus, the latter two quantities are more relevant to actual observations. Un- less otherwise stated, we will use $\langle r\rangle_{a w}$ when discussing mean partile radius. Condensation is responsible for the major abrupt increase in particle size shown in Fig. 13, and coagulation acts to continuously increase the particle size as one travels deeper into the atmosphere. Note that the particles in this model are small enough to be in the free-molecular regime throughout most of the stratosphere (e.g., the regime in which the particle radius is much smaller than the mean free path of the atmospheric gas molecules), but eventually enter the regime intermediate between the free-molecular and continuum regimes by the time they reach the lower stratosphere and troposphere.

A study of the time scales for the different loss processes (Fig. 14) helps demonstrate why the particle concentration and size profiles exhibit those shapes. In Fig. 14 , we use the mean radius $\langle r\rangle$ and the concentration profiles shown in Figs. 12 and 13 to roughly calculate the loss time scales for the haze particles in Model A. At high altitudes, the atmospheric density is low, and the particles, even though they are relatively small, fall out rapidly. On the other hand, the small size and low concentration of the particles prevents coagulation from being a major factor at high altitudes. Coagulation begins to play a larger role in the condensation region and at lower altitude levels. As the atmospheric density increases, the 

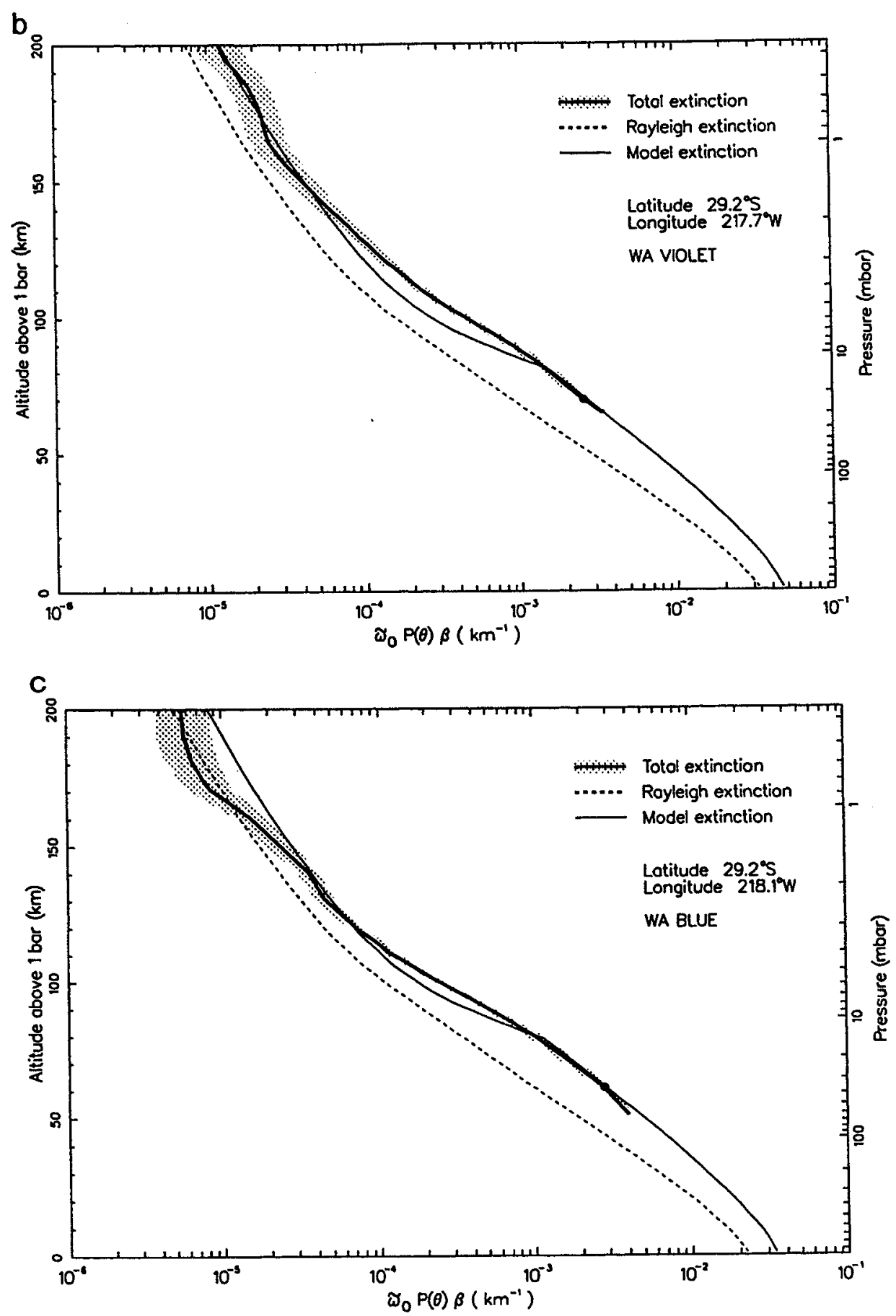

FIG. 11-Continued

sedimentation time scale for the particles becomes long, and sedimentation is no longer effective. As a result, eddy diffusion begins to dominate the vertical removal of particles, and the particle concentration becomes nearly constant in the lower stratosphere as eddy diffusion effciently removes particles to lower altitudes. Note that the particles can remain in the lower stratosphere for several years. As mentioned in Section 3.2, horizontal transport in the zonal (east-west) direction is expected to be rapid, but transport in the meridional (north-south) direction may take more than a full Neptune year.

The Model A opacities in the different Voyager filters 

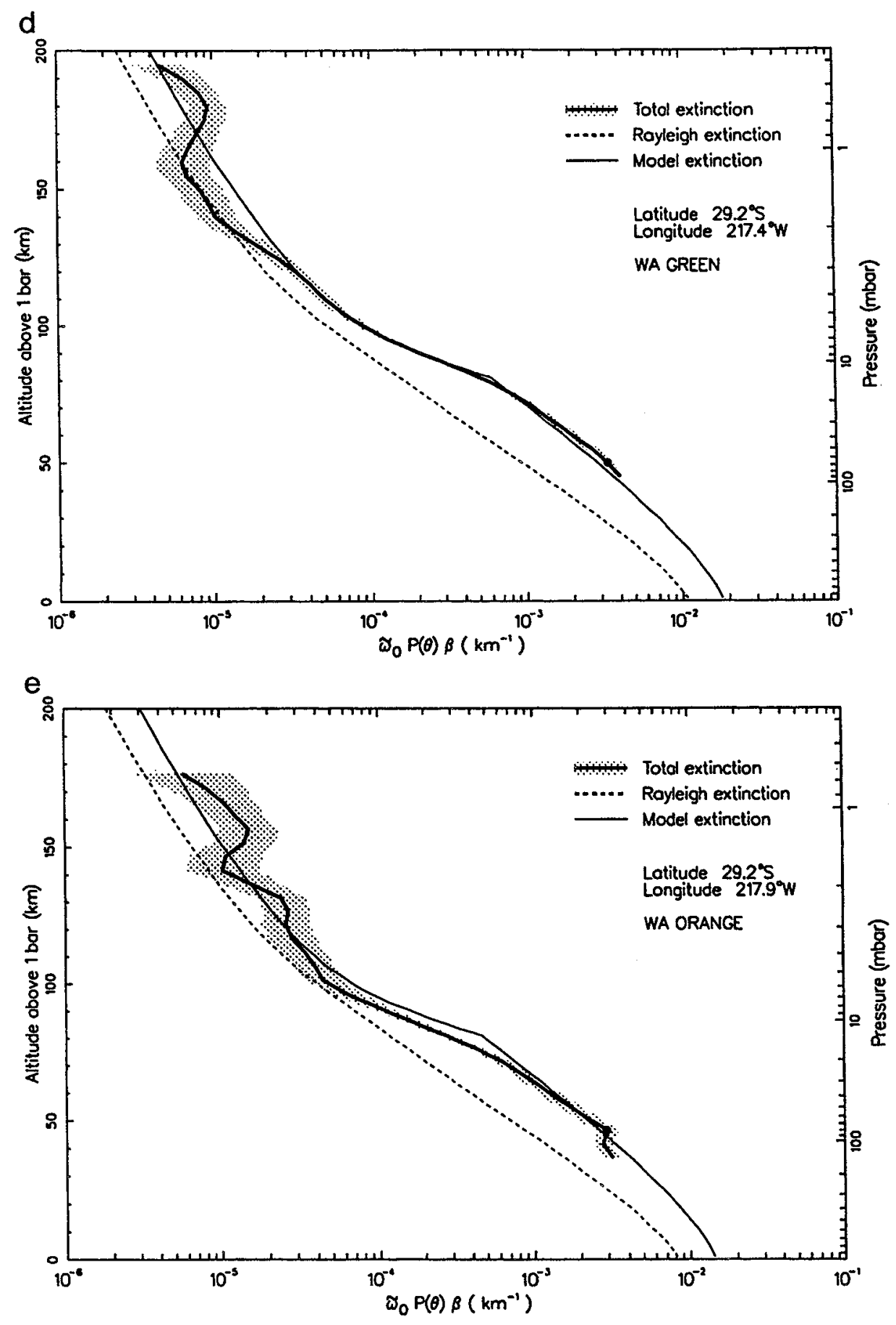

FIG. 11-Continued

are shown in Fig. 15. Remember that the high-phaseangle-observations extend down to at most $80 \mathrm{mbar}$ (with the orange filter); thus, the values we calculate for the lower altitudes (pressures higher than 80 mbar) are dependent on our model and do not result directly from the observations. If methane is oversaturated in Neptune's stratosphere, then methane might also condense in the lower stratosphere (somewhere between 40 and 100 mbar) and might affect the particle population. Methane condensation has not been included in our aerosol models because there is no evidence for a methane condensation region in the orange- and green-filter observa- 


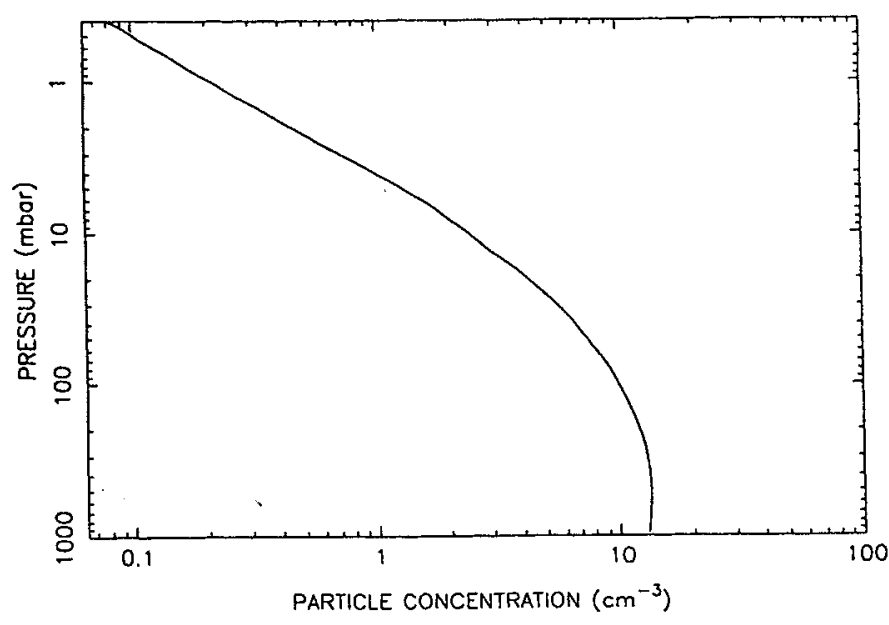

FIG. 12. The total concentration of haze particles in the best-fit aerosol model (Model A).

tions. However, we lose sensitivity at these low altitudes because the atmospheric slant path is becoming optically thick. Methane condensation cannot be ruled out from the observations. If methane is oversaturated to a large extent (e.g., greater than a factor of 2 ), then we might expect methane condensation to wash out some of the aerosol particles; in other words, the highly abundant methane would condense rapidly about the haze particles, the particles would then grow rapidly, and sedimentation would act to remove the aerosols from the lower stratosphere and upper troposphere. If this is the case, then the cumulative opacities and column mass abundances that we quote in the troposphere might be somewhat high.

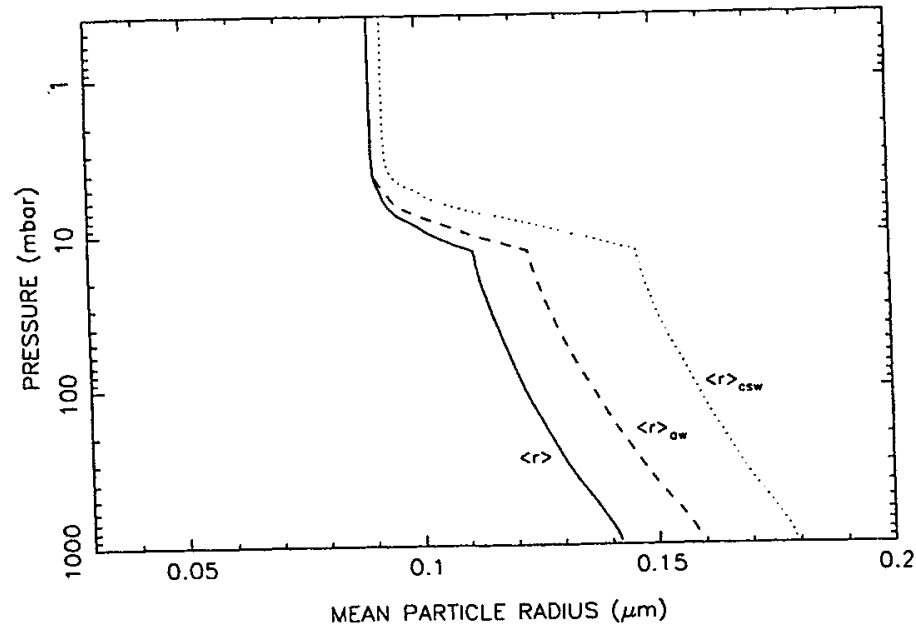

FIG. 13. The mean radius (solid line), area-weighted mean radius (dashed line), and cross-section-weighted mean radius (dotted line) in our best-fit aerosol model (Model A).

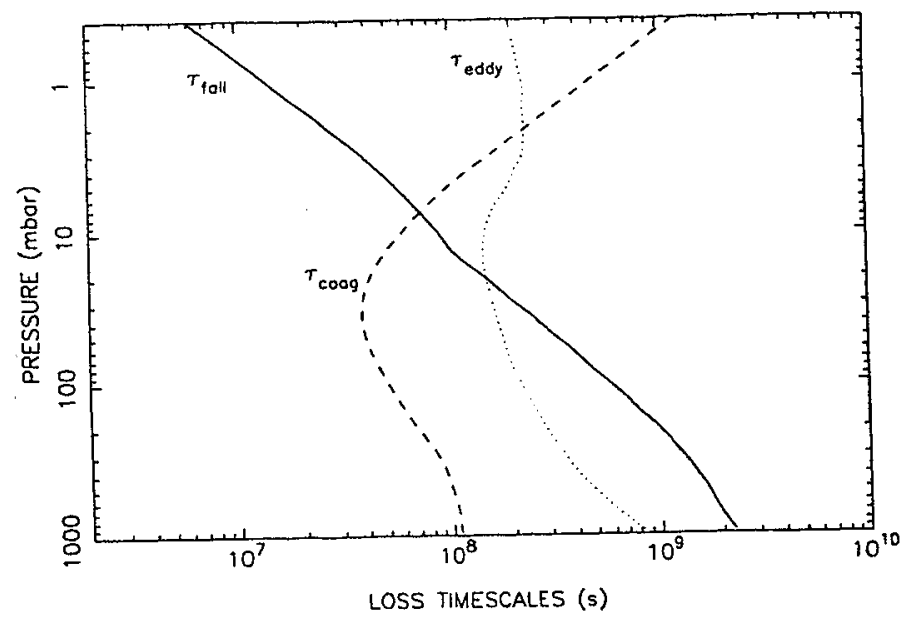

FIG. 14. The loss times scales for various processes in Model A. The coagulation time scale (dashed line) is calculated for same-sized particles with an altitude-variable mean radius as shown in Fig. 13. The sedimentation time scale (solid line) is also calculated using the meanradius profile. The eddy diffusion time scale is estimated simply as the square of the scale height divided by the eddy diffusion coefficient at each altitude.

\subsection{Sensitivity to Free Parameters}

Although Model A provides an acceptable fit to the high-phase-angle data, the sheer number of free parameters involved in the aerosol modeling makes it clear that many other physically reasonable solutions might be possible. In an attempt to place some constraints on the

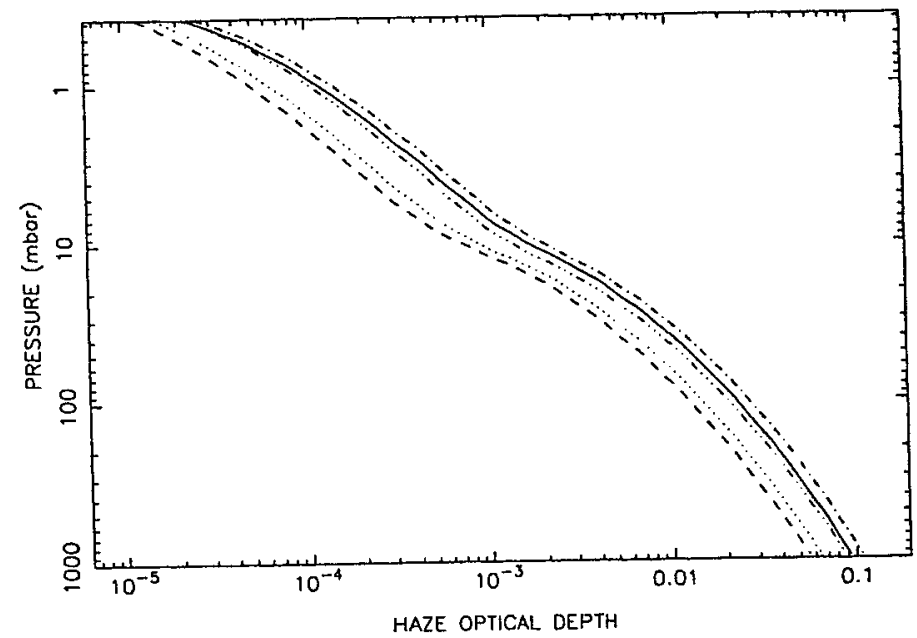

FIG. 15. Haze extinction optical depth from Model A (calculated in the vertical direction). From left to right, the dashed line is for the orange filter, the dotted line is for the green filter, the dash-double-dot line is for the blue filter, the solid line is for the clear filter, and the dotdash line is for the violet filter. The results are fairly accurate above $\sim 80$ mbar provided that our absolute altitude scale is correct. The results below 80 mbar are more model dependent. 
model parameters and results, we now examine the sensitivity of the modeling to the various free parameters. Table VII provides a summary of the free parameters and their qualitative effects on the model. Below, we discuss each of the free parameters in turn and place limits on their possible values. The error bars that we quote are approximate; we simply give the range of values that allow us to derive a reasonable fit to the observations.

Condensation flux. The single most important parameter in our aerosol modeling is the condensation flux. Although coagulation, sedimentation, and diffusion affect the particle population, condensation is the primary mechanism for controlling the amount of mass present at any altitude level. If the condensation flux is too high, either the particles will be too large or the total number of particles at any level will be too high, causing the calculated extinction coefficient and phase function to exceed the observations. We have examined models that have total condensation fluxes that range from $0.5-7 \times 10^{-15} \mathrm{~g}$ $\mathrm{cm}^{-2} \mathrm{sec}^{-1}$.

For ethane condensation fluxes similar to those predicted from photochemical models (e.g., greater than $5 \times$ $10^{-15} \mathrm{~g} \mathrm{~cm}^{-2} \mathrm{sec}^{-1}$ according to our models and those of Romani et al. 1993), the total aerosol mass in the ethane condensation region is high, and it is difficult to develop models that have the relatively small extinction coefficient and phase function that are observed in the highphase-angle Voyager images. We did find that by starting with a very small creation flux (i.e., by starting with a very small number of particles), we were able to develop models that fit one or more of the different filter observations when we used high total condensation fluxes similar to those of our standard photochemical model, but the results at the other wavelengths were always unreasonable. In fact, we found that we had to reduce the total condensation flux to $1_{-0.3}^{+0.2} \times 10^{-15} \mathrm{~g} \mathrm{~cm}^{-2} \mathrm{sec}^{-1}$ in order to reproduce the observations in all the Voyager filters.

The different wavelength filters sense different altitude regions. Because of the various physical processes operating on the particles, these different altitude regions may have different particle populations. In addition, the aerosol phase function is sensitive to $2 \pi r / \lambda$, where $\lambda$ is the wavelength; thus different wavelengths will have different observed phase functions even if the particle size distribution is similar at the different altitudes. The total phase function (see Eq. [2]) determined directly from the high-phase-angle observations is remarkably similar for all the filters (see Table VI); the values cluster around $\sim 2.6$ (corresponding to an aerosol phase function in the bullet regions of $\sim 4.5$ ). Several situations could produce this result. First, the particles could be fairly small $\left(\langle r\rangle_{\mathrm{aw}} \leqslant 0.15 \mu \mathrm{m}\right)$ in the condensation region with particle size increasing slowly toward higher pressures. As the wavelength is increased, the Rayleigh extinction coefficient decreases, and light penetrates to deeper altitudes. If the particles are relatively small, small changes in $x=$ $2 \pi r / \lambda$ with altitude or wavelength do not drastically change the aerosol phase function. Model A typifies such a solution. Another possibility is that the particles might be large $\left(\langle r\rangle_{\mathrm{aw}} \geqslant 0.5 \mu \mathrm{m}\right)$ with the particle size increasing more dramatically at higher pressures. Particles of this size are very forward scattering at visible wavelengths. Small changes in $x=2 \pi r / \lambda$ lead to dramatic changes in the aerosol phase function at any constant scattering angle. For the total phase function to then be constant with an increase in wavelength, the wavelength increase must be balanced by an increase in the particle size (e.g., $x \approx$ constant). Particle sizes in the $\langle r\rangle_{\mathrm{aw}}=0.2$ to $0.5 \mu \mathrm{m}$ range are difficult to reconcile with observations because they imply fairly large phase functions at a $20^{\circ}$ scattering angle.

Our results for a model that has a high condensation flux (hereafter called Model B) and large particles are shown in Fig. 16. We assume in Model B that small particles with radius $0.034 \mu \mathrm{m}$ are created at high altitudes (0.1-0.001 mbar) with a creation flux of $1 \times 10^{-18} \mathrm{~g} \mathrm{~cm}^{-2}$ $\mathrm{sec}^{-1}$. The assumed total haze production rate is $6.6 \times$ $10^{-15} \mathrm{~g} \mathrm{~cm}^{-2} \mathrm{sec}^{-1}$. This value is more typical of the photochemical model results than is the condensation flux from Model A. The average charge on the particles is assumed to be $1 e^{-}$per micrometer, and the mean bulk density is again calculated by taking the mass-fluxweighted average of the assumed higher density $(2.8 \mathrm{~g}$ $\left.\mathrm{cm}^{-3}\right)$ high-altitude particles and the lower density $(0.7 \mathrm{~g}$ $\mathrm{cm}^{-3}$ ) hydrocarbon ices. Note that the resulting average particle bulk density in Model B is much lower than that in Model A because Model B has a much higher rate of hydrocarbon ice condensation.

Model B illustrates that aerosol models that produce a small number of large particles in the hydrocarbon condensation regions can fit the clear-filter observations. However, such models also tend to overestimate the extinction in the longer wavelength Voyager filters (see Fig. 16b). The problem with Model B can easily be explained by an examination of the effect of particle size on the aerosol phase function. Figure 17 is a plot of the polar scattering diagrams for Models A and B, both of which fit the clear-filter observations. The biggest difference between the two models is that the condensation flux and mean particle size are smaller in Model $A$ than in Model B. Model A has a mean particle radius $\langle r\rangle_{\text {aw }}$ of $0.127 \mu \mathrm{m}$ in the clear-filter bullet region and a mean radius $\langle r\rangle_{\text {aw }}$ of $0.134 \mu \mathrm{m}$ in the orange-filter bullet region. These small particles exhibit an aerosol phase function that has a fairly broad forward peak: the phase function at a $\sim 20^{\circ}$ scattering angle (relevant to the observations) is only slightly smaller than the value at the $0^{\circ}$ peak. Model B, on 

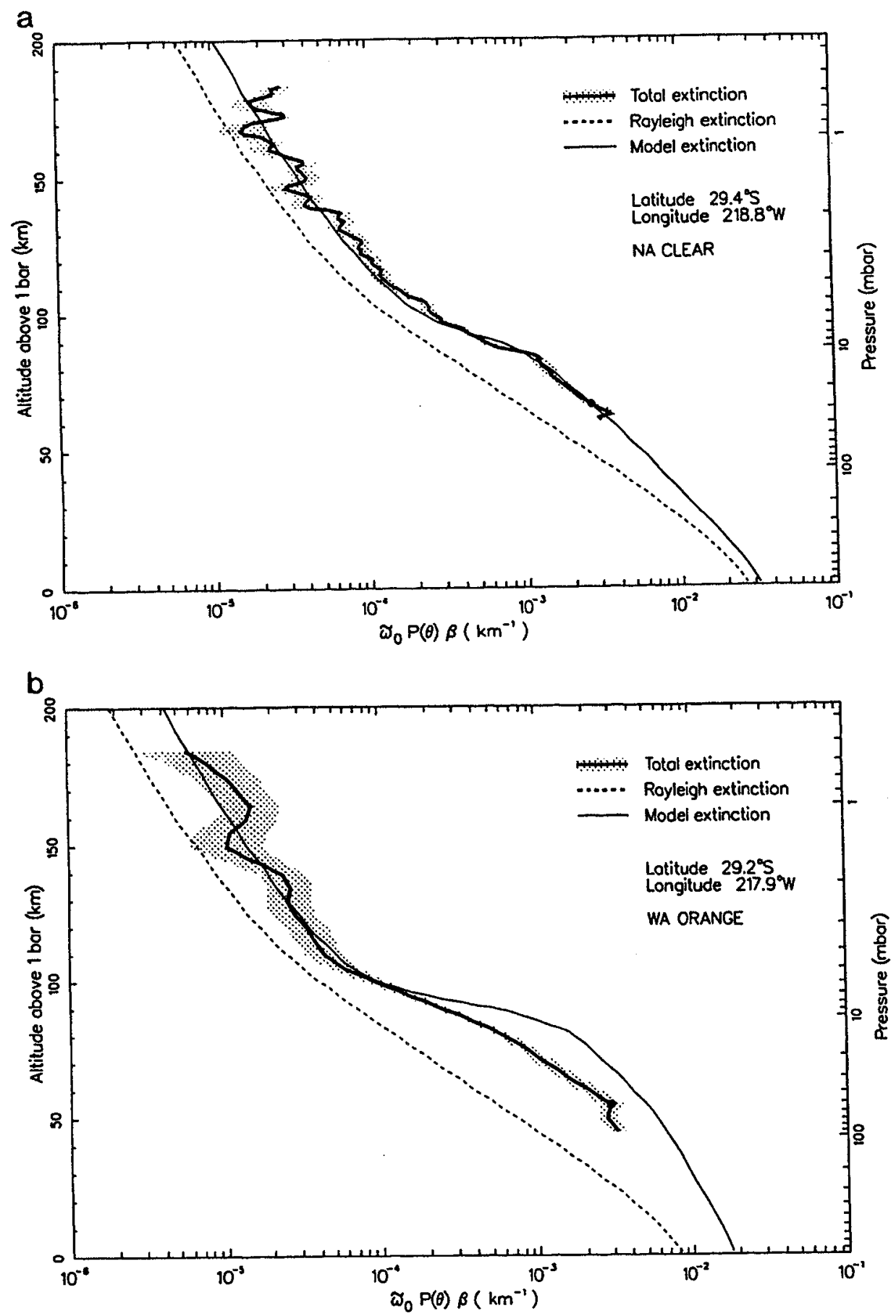

FIG. 16. A comparison of Model B with (a) the NA clear-filter and (b) the WA orange-filter observations.

the other hand, has a condensation flux similar to our standard photochemical model, $6.6 \times 10^{-15} \mathrm{~g} \mathrm{~cm}^{-2} \mathrm{sec}^{-1}$, and has much larger particles. The mean radius $\langle r\rangle_{a w}$ at the bullet region in both the clear-filter and orange-filter simulations in Model B is $0.64 \mu \mathrm{m}$. The forward peak for these large particles is very pronounced, and $20^{\circ}$ is far enough past the main $0^{\circ}$ peak that the phase function again becomes small. Both of the models have total phase functions that are near 2.5 at the bullet region of the clear-filter data; however, only Model A accurately reproduces the orange-filter data. The phase function for the Model B particles at orange-filter wavelengths is 

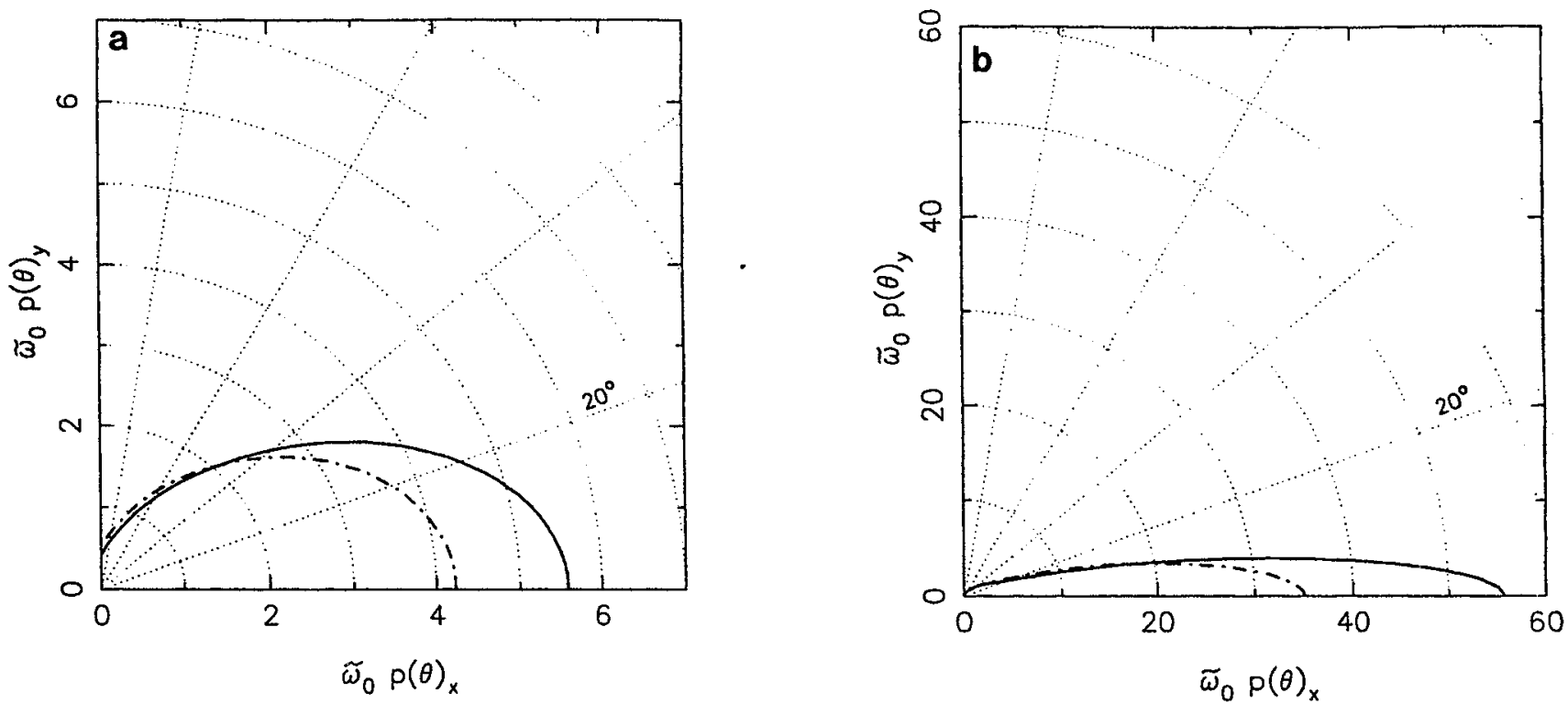

FIG. 17. Polar scattering phase diagrams for (a) Model A, and (b) Model B. The aerosol phase functions $\bar{\omega}_{0} p(\theta)$ for the particles in the bullet regions of clear-filter (solid line) and orange-filter (dot-dash line) simulations are plotted in a polar diagram with $0^{\circ}$ scattering angle along the $x$ axis and $90^{\circ}$ scattering angle along the $y$ axis. Note that the phase function peaks at $0^{\circ}$ scattering angle, and that larger particles (as in $\cdot M o d e l ~ B$ ) have forward peaks that are narrower but larger in magnitude.

broader than that at clear-filter wavelengths, and the aerosol phase function ends up being fairly large at a $20^{\circ}$ scattering angle. The total phase function (aerosol plus Rayleigh) for the large Model B particles at orange-filter wavelengths is nearly 4.6 , much higher than is observed in the high-phase-angle orange-filter images.

Creation flux and size of newly created particles. Together, the creation flux and the size of the newly created particles at high altitudes help control the concentration of aerosol particles in the model. To decrease the particle number density in the lower stratosphere, we can decrease the creation flux and/or increase the size of the newly created particles. A fairly strict constraint on the model is that the aerosol particles must be large (mean radius $\langle r\rangle_{\mathrm{aw}}=0.1_{-0.02}^{+0.01} \mu \mathrm{m}$ ) in the 0.1 - to 5 -mbar region. The particles could become this size by directly nucleating as large particles at high altitudes or by initially nucleating as small particles followed by later growth due to the condensation of a relatively abundant vapor species at pressures less than 0.1 mbar (e.g., water from meteoroid ablation). If the former method is responsible for the large particle sizes at high altitudes (as in Model A), then the creation flux of the high altitude particles will need to be large $\left(7_{-2}^{+3} \times 10^{-16} \mathrm{~g} \mathrm{~cm}^{-2} \mathrm{sec}^{-1}\right)$ to get enough extinction in the model. If the latter method is operating to increase the high-altitude particle size (as in Model B), then we have no firm constraints on the creation flux and size of newly created particles, but we can place some constraints on the flux of the vapor species that is con- densing about the aerosol particles at high altitudes. We find that condensation flux must be comparable to the creation flux quoted for the former method, $7 \times 10^{-16} \mathrm{~g}$ $\mathrm{cm}^{-2} \mathrm{sec}^{-1}$ (with a larger estimated uncertainty). The major drawback of the former method is that it is difficult to imagine that particles forming directly from a vapor state could quickly produce such large particles. The major drawback of the latter method is that it is difficult to imagine that some other as yet undetected species such as water could be abundant enough to condense at such a large rate in Neptune's upper atmosphere.

Material bulk density. The material bulk density affects the rates of coagulation and sedimentation. The higher the bulk density, the faster the particles fall, and the fewer the particles that are present at any particular altitude; thus, the smaller the extinction coefficient. On the other hand, high bulk densities imply lower coagulation rates, and the particles may remain small enough that sedimentation does not become important. The first effect dominates at high altitudes. Depending on the condensation flux and the particle size, the second effect may dominate at lower altitudes. The bulk density also affects the particle size during condensation in the model and the particle concentration during the creation of new particles in the model. For instance, if the creation flux and size of newly created particles are fixed, then the density will affect how many particles of that size can be produced at high altitudes. Since the bulk density is so highly coupled with the other parameters such as the 
condensation and creation fluxes, it is difficult to place firm constraints on this value. We find that higher average densities $\left(\langle\rho\rangle \geqslant 1 \mathrm{~g} \mathrm{~cm}^{-3}\right)$ tend to improve the fit in models similar to Model $\mathrm{A}$, where the assumed condensation flux is low $\left(\sim 1 \times 10^{-15} \mathrm{~g} \mathrm{~cm}^{-2} \mathrm{sec}^{-1}\right)$.

Eddy diffusion coefficient. A high eddy diffusion coefficient $\left(\geqslant 1 \times 10^{5} \mathrm{~cm}^{2} \mathrm{sec}^{-1}\right)$ in the condensation region helps move the particles vertically both up and down and allows smoother profiles of the extinction coefficient and albedo-weighted phase function. A small diffusion coefficient $\left(\leqslant 1 \times 10^{4} \mathrm{~cm}^{2} \mathrm{sec}^{-1}\right)$ in this region allows sharp changes in particle size with altitude as condensation occurs; thus, both $\beta$ and $\tilde{\omega}_{0} p(\theta)$ will exhibit abrupt increases in the condensation region. The shorter wavelength data favor smoother profiles and higher diffusion coefficients while the longer wavelength data favor more abrupt condensation regions and lower diffusion coefficients in the condensation region. Since it appears that we cannot simultaneously fit both the violet- and orange-filter data with any of our choices of the diffusion coefficient, we use a compromise value of $K \sim 3 \times 10^{4} \mathrm{~cm}^{2} \mathrm{sec}^{-1}$ at 12 mbar. When we use the Case $C$ diffusion profile of Romani et al. (1993) illustrated in Fig. 4, but leave the other parameters the same as in Model A, we get results as in Fig. 18. The fit in the violet and clear filters is slightly worse than that in Model A, but the fit in the orange filter is better.

A large value of the diffusion coefficient at the lower boundary causes particles to be rapidly lost to the troposphere. The choice of a large diffusion coefficient near our 1-bar lower boundary hurts the fit at all wavelengths. We find that $K \leqslant 1 \times 10^{4} \mathrm{~cm}^{2} \mathrm{sec}^{-1}$ at our lower boundary is necessary to obtain a good fit between models and data, but the constraints on the actual value are not particularly strong. Note that the temperature profile in Neptune's upper troposphere as measured by Lindal (1992) is subadiabatic at 1 bar and higher altitudes; thus, it is not unreasonable to assume that the eddy diffusion coefficient is low in this region. Lower down, as the profile becomes adiabatic and the atmosphere freely convective, one might expect a higher eddy diffusion coefficient (Stone 1976).

Stratospheric temperature profile. The stratospheric temperature profile controls the atmospheric density variation with altitude, which in turn controls the amount of Rayleigh scattering at any altitude. The adoption of a different temperature profile can change our results dramatically, especially at high altitudes. However, we have constraints on the temperature profile from ground-based and Voyager observations (see Appendix A). If we were to adopt the RSS profiles (either ingress or egress) for the troposphere and lower stratosphere and use the same middle stratospheric temperature that we are currently using, then the atmosphere would be less dense in the 0.1 - to 10-mbar region than our current model, and even larger particles would be required at high altitudes to match the observations (e.g., mean radii of $\langle r\rangle_{\mathrm{aw}} \geq$ $0.13 \mu \mathrm{m})$.

In order to increase the atmospheric density by the factor of $\sim 2$ that is necessary at any altitude to have the high-altitude observations follow the Rayleigh extinction curve, we would need a large increase in the average temperature below the 0.1 -mbar region. Such an increase cannot come from the high-altitude regions alone; e.g., a factor of 2 increase in the temperature in the 1-mbar region is not supported by observations. However, a smaller increase in the temperatures at all pressure regions, including the upper troposphere and lower stratosphere, might produce the desired effect. Since the profile that we use (from Orton et al. 1992) is already much warmer than the RSS profiles, we hesitate to increase the temperature any further. In fact, the location of the major condensation level (12 mbar) in the observations suggests that either our current profile is too warm in the 12 -mbar region or that we have underestimated the ethane density in our standard model, or perhaps both. In addition, our hydrogen density in the 0.2 - to $0.4-$ mbar region matches the UVS observations of Yelle et al. (1993; see Fig. 3). Thus, we believe that our background atmosphere (i.e., altitude-density profile) is not uncertain to within a factor of 2 in density, and we believe that the high-phase-angle images do support the conclusion that relatively large particles are present at high altitudes on Neptune. Errors in our absolute altitude scale simply cannot resolve the problem.

Charge. The amount of charge on the particles affects the coagulation rate-coagulation is inhibited if the particles are similarly charged with a high charge concentration per unit length (radius). Therefore, the amount of charging on the particles will affect the particle size distribution. In our models, however, the charging does not appreciably affect the total mass of condensed material at any altitude; it just controls the size and concentration of the same amount of mass. The extinction coefficient is thus not measurably affected by small changes in the charge concentration, but the albedo-weighted phase function, which is more sensitive to the particle size, can notably increase or decrease with a factor of 2 change in the value of the charge concentration. For every fixed value of the creation flux, size of newly created particles, condensation flux, and average particle bulk density, there is only one value of the charge that will reproduce both the extinction coefficient and phase function in the bullet region of the observations; however, the actual value of the charge is to dependent on the other parameters for us to place any useful constraints on its magnitude. 


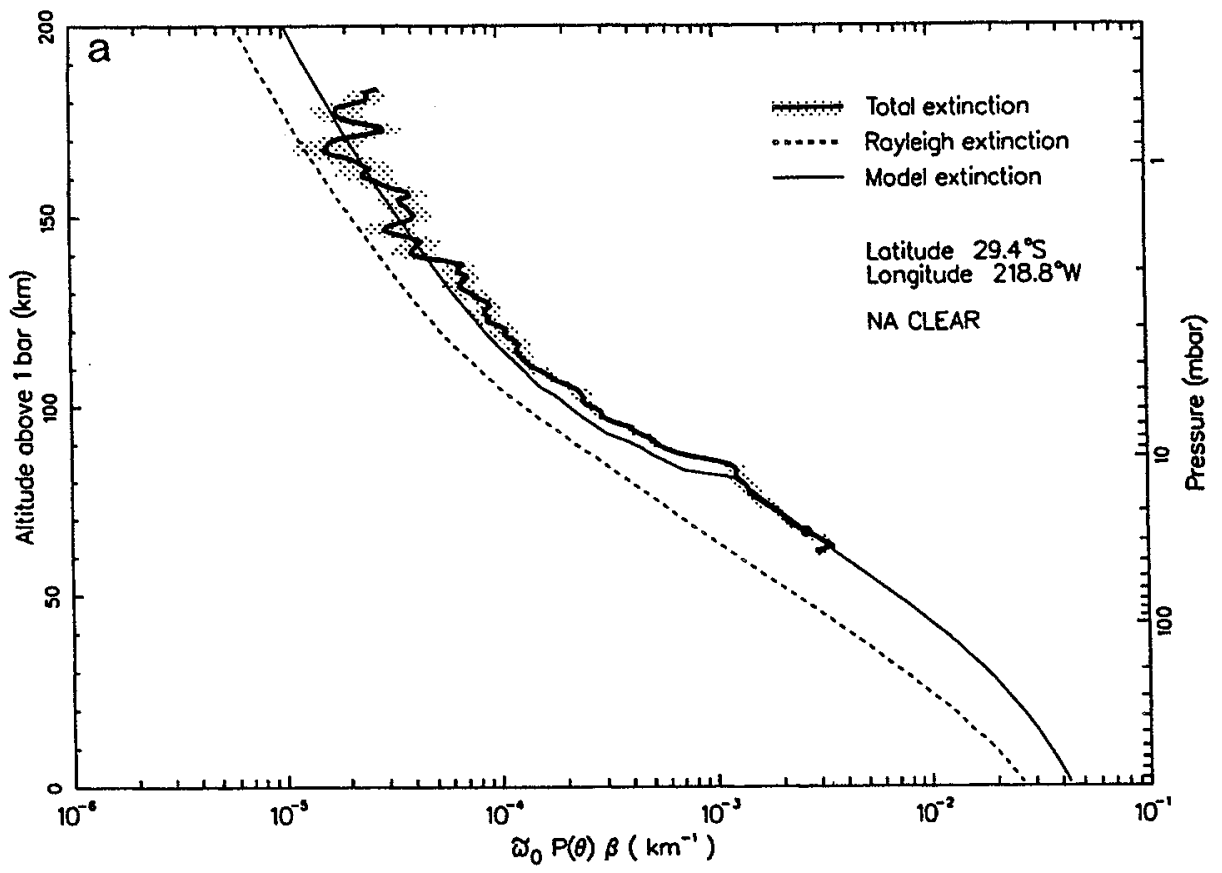

FIG. 18. A comparison of an aerosol model that uses the Case $C$ eddy diffusion coefficient of Romani et al. (1993) and the Model $A$ input parameters with (a) the NA clear-filter, (b) the WA violet-filter, and (c) the WA orange-filter observations.

Condensation levels. If our adopted temperature profile is correct, then the narrow-angle camera clear-filter observations indicate that greater than $70 \%$ of the total condensation in Neptune's stratosphere occurs at $12_{-1}^{+1}$ mbar. Aside from this major condesnation level, which is most likely due to ethane condensation, the actual locations of the other condensation levels have little effect on the results. If the eddy diffusion coefficient is high $(\geq 1 \times$ $\left.10^{4} \mathrm{~cm}^{-2} \mathrm{sec}^{-1}\right)$ in the condensation region, then there is no abrupt observable increase in extinction at the other condensation levels; we cannot constrain the relative haze production rates at pressures other than 12 mbar.

Optical properties. Changing the optical properties within reasonable limits can cause a few percent change in the calculated phase function and extinction coefficient. The results are more sensitive to the value of the real refractive index than to that of the imaginary refractive index because scattering, rather than absorption, dominates the extinction profile. Since most of the condensation in Neptune's lower stratosphere is due to ethane, the real refractive index of the hazes should be very close to 1.44 , at least in the outer layers of the aerosol particles. If some other material (such as methane) contributes greatly to the optical properties, then the total extinction coefficient times phase function might change by as much as $-30 \%$. The choice of the real index of the core material (e.g., ices vs silicates vs kerogen) can have a few percent effect on the results but will not change our conclusions. We cannot constrain the wavelength dependence of the imaginary refractive index of the hazes using this high-phase-angle data set.

\subsection{Comparisons with Other Observations}

The Voyager images are not the only observations that provide us with information about the stratospheric hazes on Neptune. Quantitative information is also derived from other ground-based and Voyager observations at ultraviolet, visible, and near-infrared wavelengths. Evidence for stratospheric hazes on Neptune has been documented for many years (see review by Baines et al. 1994). Several recent analyses of Earth-based (Baines and Hammel 1994) and Voyager (Pryor et al. 1992) data have provided estimates for the mean particle size, total haze column abundance, and haze production rate in Neptune's stratosphere.

Analyses of ground-based observations are sensitive to assumptions about certain unresolved properties of Neptune's atmosphere (such as the methane abundance in the stratosphere). As a result, the haze altitude distribution and total column abundance inferred from ground-based observations should be regarded as somewhat model dependent. Recent results reported by Baines and Hammel (1994) suggest that stratospheric aerosols have a total inferred column mass of $0.2-1.5 \times 10^{-6} \mathrm{~g} \mathrm{~cm}^{-2}$ if the stratospheric methane mixing ratio is near $3.5 \times 10^{-4}$ and if the particles have mean radii $\langle r\rangle$ of $0.2 \mu \mathrm{m}$. Baines and 

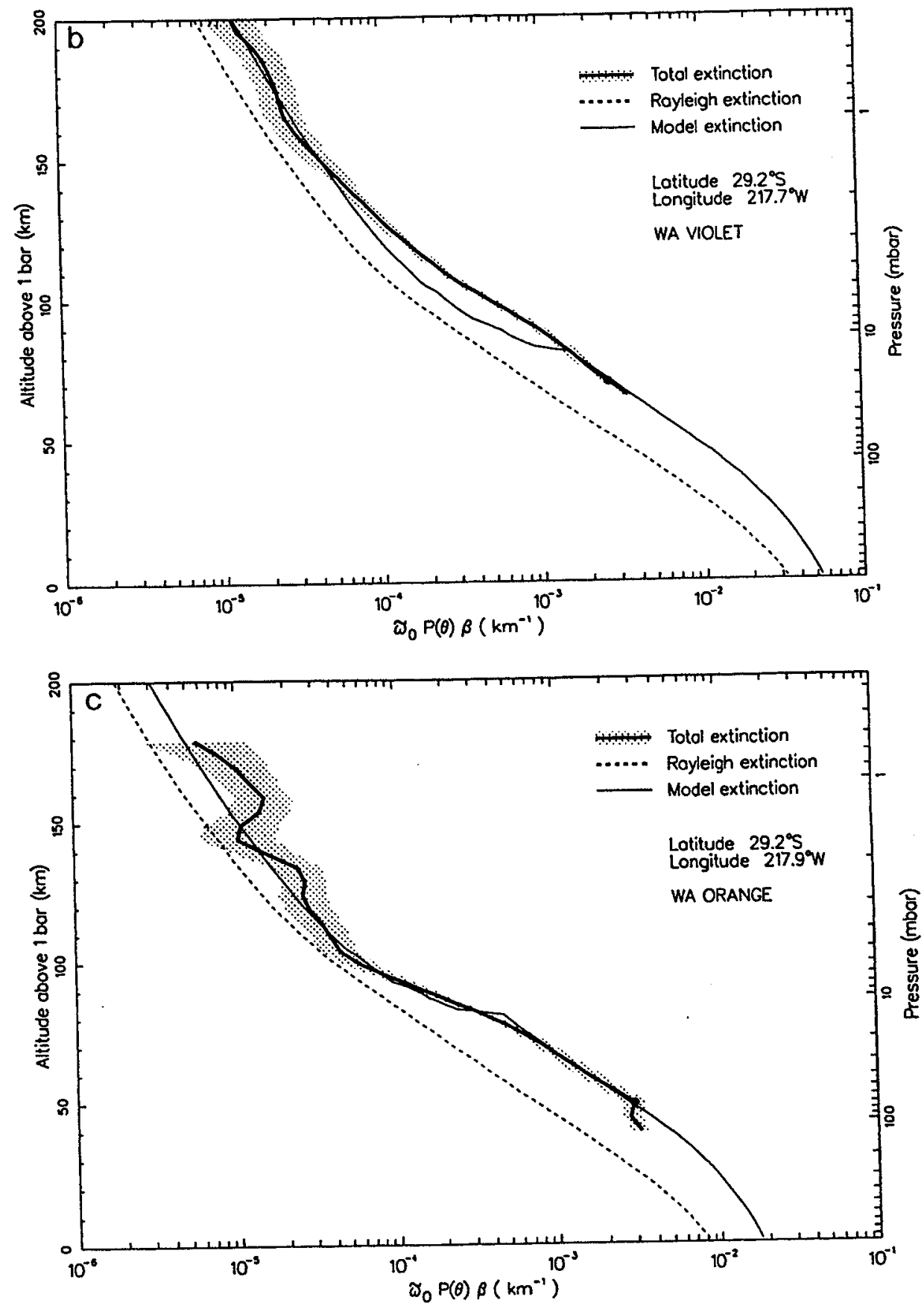

FIG. 18-Continued

Hammel indicate that the range in the above quotes for column abundance represents real differences in their results for the 1986 "equatorial region" versus the 19811986 "global mean" for Neptune. Their results demonstrate that the stratospheric aerosol abundance on Neptune can vary with location on the planet (e.g., latitude region) and/or with time (e.g., the year of the observations).
Table VIII shows how the Baines and Hammel (1994) results compare with ours. In this table, we present the column mass abundance of haze particles from our bestfit Model A above a few particular pressure levels, show the mean radius and area-weighted mean radius at these levels, and present the optical depths at these levels for the NA-camera clear filter and two monochromatic wavelengths, 0.265 and $0.750 \mu \mathrm{m}$. Baines and Hammel 
TABLE VIII

Properties of Model A Compared with Other Observations

\begin{tabular}{|c|c|c|c|c|c|c|c|}
\hline $\begin{array}{l}\text { Pressure } \\
\text { (mbar) }\end{array}$ & $\begin{array}{l}\text { Col. mass } \\
\left(\mu \mathrm{g} \mathrm{cm}^{-2}\right)\end{array}$ & $\begin{array}{c}\langle r\rangle \\
(\mu \mathrm{m})\end{array}$ & $\begin{array}{l}\langle r\rangle_{\mathrm{aw}} \\
(\mu \mathrm{m})\end{array}$ & $\stackrel{{ }^{\top}}{\text { (clear filter) }}$ & $\begin{array}{c}\tau \\
(0.265 \mu \mathrm{m})\end{array}$ & $\underset{(0.75 \mu \mathrm{m})}{\tau}$ & Reference \\
\hline 4.9 & 0.021 & 0.091 & 0.092 & 0.00068 & 0.0028 & 0.00012 & This work" \\
\hline 14 & 0.064 & 0.112 & 0.123 & 0.0029 & 0.0088 & 0.00065 & " \\
\hline 40 & 0.18 & 0.116 & 0.129 & 0.010 & 0.024 & 0.0026 & $\ddot{ }$ \\
\hline 110 & 0.38 & 0.123 & 0.137 & 0.024 & 0.050 & 0.0067 & $"$ \\
\hline 870 & 1.3 & 0.141 & 0.159 & 0.088 & 0.15 & 0.028 & " \\
\hline 20 & 1.5 & 0.2 & & & 0.37 & 0.13 & $\begin{array}{l}\text { Baines and } \\
\text { Hammel }(1994)^{h}\end{array}$ \\
\hline 20 & 0.20 & 0.2 & & $0.035^{d}$ & 0.048 & 0.017 & $\begin{array}{l}\text { Baines and } \\
\text { Hammel (1994) }\end{array}$ \\
\hline 15 & 0.1 & & 0.2 & & 0.02 & & $\begin{array}{l}\text { Pryor et al. } \\
\quad \text { (1992) }\end{array}$ \\
\hline 100 & 1.0 & & 0.25 & $0.11^{d}$ & 0.19 & 0.05 & $\begin{array}{l}\text { Pryor et al. } \\
\quad(1992)\end{array}$ \\
\hline
\end{tabular}

a Note that the aerosol properties derived from this work are based on observations that are only reliable down to $\sim 40$ mbar. The results at higher pressures are based on the aerosol-dynamics model and are conjectural.

b 1981-1986 global mean values.

c 1986 equatorial band values.

The clear-filter optical depths are not given in Baines and Hammel (1994) or Pryor et al. (1992) but have been reconstructed based on the descriptions of their aerosol models.

(1994) have assumed that the stratospheric aerosols lie between 1.4 and 20 mbar. According to our aerosol and photochemical models, the stratospheric haze particles will not begin to evaporate until they fall to at least 800 mbar (e.g., in the troposphere); thus, our aerosol layer is spread out over a much larger altitude region. Even so, our column mass abundance above 870 mbar is $1.3 \times 10^{-6}$ $\mathrm{g} \mathrm{cm}^{-2}$, similar to Baines and Hammel's global mean values for 1981-1986. However, much of the mass in our model is below the 116-mbar tropopause. The derived stratospheric optical depth in our model is notably smaller than the global-mean results inferred by Baines and Hammel (see Fig. 15). Note, however, that Baines and Hammel assume a mean particle radius of $\langle r\rangle=0.2$ $\mu \mathrm{m}$ while we find that particles this large cannot account for the small phase function derived from the high-phaseangle images. The total haze optical depth in our model is 0.15 at $0.265 \mu \mathrm{m}, 0.088$ in the clear filter (effective wavelength $0.47 \mu \mathrm{m}$ ), and 0.028 at $0.75 \mu \mathrm{m}$. These values are intermediate between the global-mean and equatorialband opacities inferred by Baines and Hammel (1994), but again, much of the haze opacity in our model is derived from particles below the troposphere.

Spatially resolved high-phase-angle observations in the $20^{\circ}-30^{\circ} \mathrm{S}$ latitude region of Neptune were taken at 0.265 and $0.750 \mu \mathrm{m}$ with the Voyager PPS instrument (Pryor et al. 1992). This data set is complementary to the ISS observations in the sense that the observations were located in the same latitude region, had similar phase-angle cov- erage, but were taken at different wavelengths and lack the ISS vertical resolution. The PPS observations indicate that haze particles with an area-weighted mean radius of $0.15-0.25 \mu \mathrm{m}$ exist between 5 and $100 \mathrm{mbar}$ in Neptune's stratosphere, contributing a haze optical depth of $0.19 \pm 0.08$ at $0.265 \mu \mathrm{m}$ and $0.05 \pm 0.02$ at 0.750 $\mu \mathrm{m}$. Pryor et al. (1992) claim that there is some evidence in the PPS data for the aerosols to be spread out a bit more in altitude than they assumed in their modeling, i.e., from 5 to 250 mbar instead of from 5 to 100 mbar. Assuming a particle radius of $0.2 \mu \mathrm{m}$ and a particle bulk density of $0.7 \mathrm{~g} \mathrm{~cm}^{-3}$, Pryor et al. infer a total column mass of the hazes of $0.58-1.4 \mu \mathrm{g} \mathrm{cm}^{-2}$ from the PPS data, with only $0.061-0.15 \mu \mathrm{g} \mathrm{cm}^{-2}$ deriving from the 5- to 15 mbar region. Further assuming that the sedimentation velocity for these particles is 3.9 mbar year $^{-1}$, Pryor et al. infer a stratospheric haze production rate of $1-2 \times 10^{-15} \mathrm{~g}$ $\mathrm{cm}^{-2} \mathrm{sec}^{-1}$ from high-phase-angle PPS data.

These values are again similar but somewhat larger than our Model A results (see Table VIII). The column mass abundances are similar-our model has $30 \%$ more total stratospheric mass and $\sim 50 \%$ less mass above 15 mbar than the Pryor et al. results. Our total stratospheric opacities are similar, but we find that the opacity near 15 mbar in our model is a factor of 2 smaller at $0.265 \mu \mathrm{m}$ than that derived by Pryor et al. (1992). In addition, we find that particles of area-weighted mean radii greater than 0.2 cannot fit the observations, and we favor particle sizes at 15 mbar of $\langle r\rangle_{\mathrm{aw}}+0.12 \pm 0.02$, whereas Pryor $e t$ 


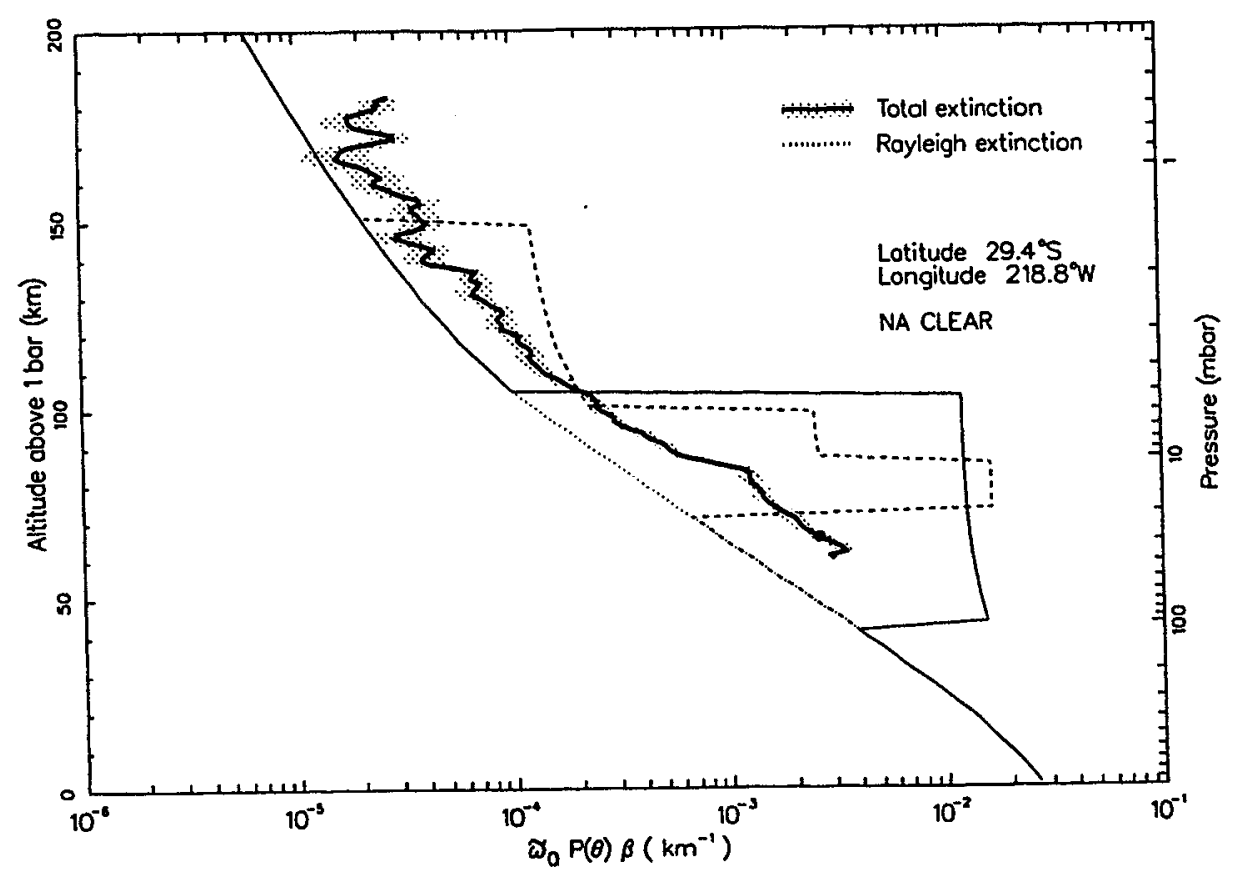

FIG. 19. A comparison of the Pryor et al. (1992) (thin solid line) and Baines and Hammel (1994) (dashed line) aerosol models with the NA clearfilter data. See Fig. 11a for a similar comparison using our aerosol Model A.

al. (1992) find that they cannot fit the high-phase-angle $0.265-\mu \mathrm{m}$ data if the particles are $\sim 0.1 \mu \mathrm{m}$ and favor particle sizes of $0.2 \pm 0.05$.

It is difficult to determine at this point whether our results are within the lower error bounds of the Pryor et al. observations or whether the two data sets are mutually exclusive. Our modeling procedures are not identical. Pryor et al. (1992) assume that the aerosols are uniformly mixed with the particle size remaining constant with altitude in the stratosphere while we find that both the concentration and particle size are increasing with decreasing altitude in the lower stratosphere (Figs. 12 and 13). We also find that the haze layer may extend to larger depths than is assumed by Pryor et al. (1992) and find that high-altitude hazes may exist, which may also affect the short-wavelength PPS results. Furthermore, our haze optical properties differ somewhat. We use a shell/mantle Mie-scattering code instead of a Mie code for homogeneous spheres and do not know the wavelength dependence of the optical properties. However, the ISS observations produce fairly strict limitations on the opacity of the hazes about 15 mbar, at least at visible wavelengths. Further work is needed before we can determine whether our results are consistent with the lower limits provided by the high-phase-angle PPS observations. In any case, both sets of observations indicate that the total production rate of aerosols in Neptune's strato- sphere is much smaller than that of the photochemical model predictions.

Figure 19 demonstrates how the Baines and Hammel (1994) and Pryor et al. (1992) aerosol models compare with our high-phase-angle limb scans. Here, we use information on aerosol column densities, particle size distributions, and optical properties provided in the two abovementioned papers to calculate the extinction that their models would exhibit at high phase angles in the Voyager clear filter. We assume that the particles are homogeneous Mie-scattering spheres with an imaginary refractive index of $3 \times 10^{-4}$ for these calculations; the results are insensitive to the exact value chosen. Note that both the Pryor et al. and Baines and Hammel models overpredict the extinction coefficient times the phase function $\bar{\omega}_{0} p(\theta) \beta_{\text {tot }}$ in the 5- to 40 -mbar region at our limb-scan site. The main problem lies with the phase function $\bar{\omega}_{0} p(\theta)_{\text {haz }}$, but the extinction coefficient $\beta_{\text {haz }}$ is also too large, making $\bar{\omega}_{0} p(\theta)_{\text {tot }}$ too large. Here, again, is evidence that the particles at our site are smaller than 0.2 $\mu \mathrm{m}$ and/or that the aerosol concentrations derived by the previous studies are too high in this altitude region. Note also that both models assume stratospheric aerosols reside in narrowly confined altitude regions; our highphase-angle information suggests that particle properties vary more smoothly with altitude and that scattering particles extend at least as high as 0.6 mbar and as low as $\mathbf{8 0}$ 
mbar (orange-filter information). It will be interesting to determine through further studies whether aerosol distributions similar to our Model A (with extended amounts of smaller particles) can be consistent with the groundbased and PPS data.

\subsection{Comparisons with Uranus}

The total haze production rate on Uranus was found to be $\sim 1.0 \pm 0.1 \times 10^{-16} \mathrm{~g} \mathrm{~cm}^{-2} \mathrm{sec}^{-1}$ (Rages et al. 1991). Eddy diffusion coefficients are higher on Neptune than on Uranus, methane reaches higher altitudes on Neptune than on Uranus, and photochemical models predict higher production rates of higher-order hydrocarbons on Neptune than on Uranus (Romani et al. 1993, Moses et al. 1992). Thus, we expect more condensible hydrocarbons like ethane and acetylene to be present on Neptune than on Uranus. In fact, observations of these two species on Uranus (Herbert et al. 1987, Orton et al. 1990, Bishop et al. 1990) indicate gas mixing ratios of $\sim 1 \times$ $10^{-8}$ at 0.1 mbar for both $\mathrm{C}_{2} \mathrm{H}_{2}$ and $\mathrm{C}_{2} \mathrm{H}_{6}$, lower than those measured for Neptune (see Fig. 5). Therefore, we might expect the total haze production rate on Neptune to greatly exceed that of Uranus-theoretical photochemical models predict a full order of magnitude or more difference. That is indeed what we observe. The stratospheric haze production rate on Neptune appears to be a factor of 10 higher on Neptune than on Uranus according to the Voyager high-phase-angle imaging analyses.

However, photochemical models for Uranus predict stratospheric haze production rates of $2-4 \times 10^{-16} \mathrm{~g} \mathrm{~cm}^{-2}$ $\mathrm{sec}^{-1}$ (Summers and Strobel 1989, Rages et al. 1991), a factor of 2-4 higher than the observations. In addition, the photochemical model predictions for the condensation rates at different altitudes due to different hydrocarbon species do not compare well with observations. The Neptune models exhibit the same problems, suggesting that we need to reexamine our views of hydrocarbon photochemistry.

\subsection{Conflicts with Photochemical Models}

The major conflict between Neptune photochemical models and observations is that the models overpredict the stratospheric haze production rate by a factor $\sim 7$. How can we reconcile this problem? The photochemical predictions are fairly robust. We have some good observations of the methane, ethane, and acetylene vapor abundances on Neptune (Yelle et al. 1992, Orton et al. 1992, Kostiuk et al. 1992) that help us constrain the eddy diffusion coefficient in Neptune's middle and upper stratosphere. We have been unsuccessful in developing a photochemical model that has low downward fluxes of these species similar to those derived from the haze observa- tions but that does not violate the gas-abundance observations either in the upper or lower stratosphere. Somehow, the effective haze production rate on Neptune seems to be different from the predicted flux of the condensible vapor passing through the condensation region. How can this happen? Let's look at some of the possibilities: (a) perhaps the bulk of the vapor simply does not condense, (b) perhaps the atmosphere is not in steady state, (c) perhaps the $-30^{\circ} \mathrm{S}$ latitude region of the highphase-angle images is different from the global average or large-scale meridional motions are acting to redistribute particles to other latitudes, (d) perhaps ethane is destroyed chemically in the lower stratosphere between the lowest point of the observations and the top of the ethane condensation region, (e) perhaps the atmosphere is incredibly stagnant in the lower stratosphere, implying lower eddy diffusion coefficients than have been considered here, (f) perhaps the particles falling through the condensation regions are highly porous or fractal in nature allowing some of the condensed mass to be hidden, or (g) perhaps a few large particles are falling rapidly through the condensation region and are dominating the condensation rate but are not affecting the optical properties.

Most of the above suggestions are highly unlikely. Given that high-altitude particles exist and that ethane would be grossly supersaturated in the lower stratosphere if there were no ethane condensation, we find it extremely unlikely that option (a) could occur. Given the physical conditions encountered in Neptune's lower stratosphere, ethane and many of the other condensible hydrocarbons cannot remain highly supersaturated. Options (b) and (c) are possible but not very satisfying; we discuss option (c) in more detail below. Ethane cannot be destroyed photochemically in Neptune's lower stratosphere given our current understanding of how the photochemistry operates; thus option (d) appears unlikely. However, galactic cosmic rays, which penetrate to Neptune's lower stratosphere, might be able to break apart ethane or other molecules and might act to eventually reduce the ethane abundance by initiating the formation of other chemical species. For such a process to reduce the total haze production rate, the new species must be more volatile than ethane and must not condense in the lower stratosphere. We find this option to be improbable. The high ethane density derived from infrared observations suggests that we have overestimated eddy diffusion coefficients in the lower stratosphere. The adoption of a different eddy profile may resolve both the ethane mixing ration and flux problems. However, the UVS methane observations indicate that methane reaches high altitudes on Neptune, and it is not clear that an extremely stagnant lower stratosphere can satisfy this constraint. In any case, option (e) warrants further study. 
If the particles that are falling into the condensation region are highly fractal or porous, it is possible that much of the ethane could condense without measurably increasing the particle size, but the sheer amount of mass available for condensation makes option (f) unlikely; the particles would still grow large enough to cause inconsistencies with the observed phase functions. If a small number of large particles (e.g., $\geq 1 \mu \mathrm{m}$ radius) are falling through the condensation region as suggested by option (g), then ethane would preferentially condense about these particles. The large particles would fall out more rapidly and have phase functions that are so sharply peaked that they do not contribute substantially to the observed extinction. The large particles could be unablated meteoroids or some other foreign debris, but could not be simply the tail-end of a normal particle distribution since the presence of any particles in the radius range 0.2 to $0.5 \mu \mathrm{m}$ would likely have an effect on the optical properties. This last option is possible, but needs more study before we can suggest that it is currently operating to reduce the "effective" observed haze production rate on Neptune.

The possibility of latitudinal variations in stratospheric haze properties is certainly viable. In fact, Baines and Hammel (1994) provide evidence for such variations on Neptune in past observational years (see Table VI). Bézard et al. (1991) find evidence in high-resolution IRIS data for latitudinal variations in acetylene emission at the time of the Voyager encounter. Bézard et al. conclude that if the differences in emission are due to differences in $\mathrm{C}_{2} \mathrm{H}_{2}$ abundances with latitude, then the acetylene concentration could vary by as much as a factor of 5 across the planet. Since the acetylene haze production rate is correlated with the gas concentration, aerosol production might also be expected to vary with latitude. However, the differences observed by Bézard et al. may be due to temperature differences rather than (or as well as) concentration differences, and such temperature differences are expected on theoretical grounds (Conrath et al. 1991a). It is therefore unclear whether the IRIS observations support the possibility of latitudinally varying haze production rates. In addition, the PPS ultraviolet images (Lane et al. 1989) and the ISS visible images (Smith et al. 1989) suggest that, if anything, the latitude region probed in this study might contain more ultraviolet-absorbing, visible-scattering hazes than the bulk of the planet, not less. Although there are no quantitative numbers to back up this qualitative impression, it does seem surprising that we have derived such low condensation fluxes for this $\sim 30^{\circ} \mathrm{S}$ latitude region. More theoretical work describing stratospheric circulation patterns or unusual condensation scenarios may be required before the low apparent condensation flux can be explained.

One problem with our procedure for calculating parti- cle distributions in the aerosol-dynamics code is that we assume that new particles all start out at the same size. If we had chosen a small enough particle size and if coagulation were acting to rapidly increase this size (as occurred in the Uranus study; see Pollack et al. 1987 and Rages et al. 1991), then our choice of a monochromatic size distribution would not matter. However, we find that large particles must exist at high altitudes on Neptune; thus, we needed to begin with a large initial size. These particles do not begin to coagulate or grow until they fall to the condensation regions in the lower stratosphere and do not grow drastically even then. Therefore, the particle population tends to peak just past $0.1 \mu \mathrm{m}$, fall off gradually toward larger sizes, and fall off abruptly at small sizes (see Fig. 20). In other words, we have no particles smaller than the initial size in our model. In a real atmosphere, the high-altitude particles would exhibit a large range of sizes, causing a much broader distribution of sizes by the time the particles fall to the condensation regions. Some of the condensed ethane could be "hidden" in small particles. However, the small particles would affect the total extinction in the atmosphere, and it might still be difficult to support the predicted haze production rate even if we were to use a more realistic particle distribution. There is simply too much mass to support the observed extinction. In any case, our above conclusions should be regarded as preliminary until the discrepancy between photochemical model predictions and apparent observational constraints is better resolved.

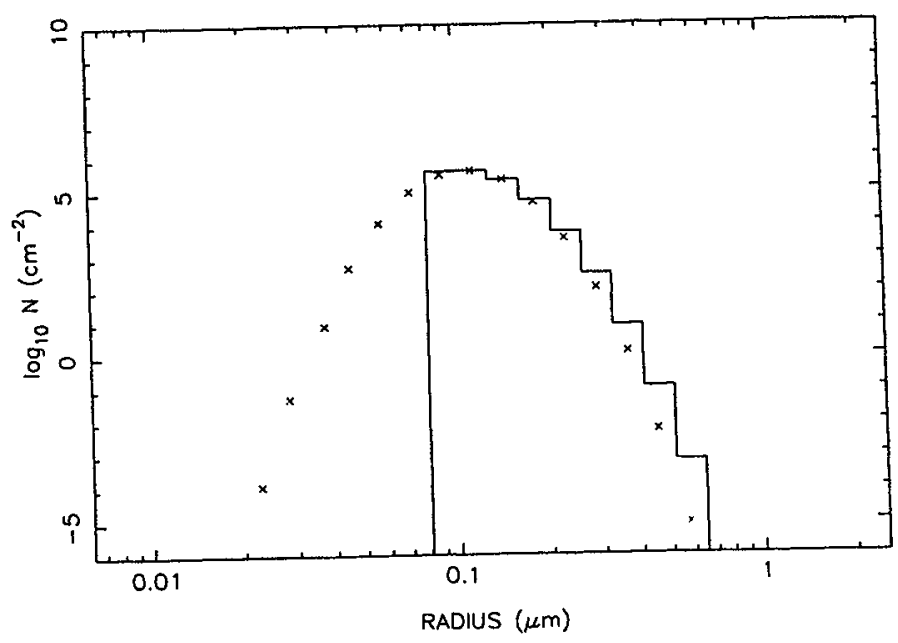

FIG. 20. The particle size distribution at 29 mbar in our Model A (solid line histograms) compared with a fitted log-normal distribution (crosses). The column abundances in the layer should be divided by the depth of the layer $(2 \mathrm{~km})$ in order to obtain particle number density. The sizes with larger particle concentrations were weighted more heavily during the fitting procedure. Log-normal distributions clearly do not fit the actual calculated particle distribution very well. The fit is much worse at lower pressures (higher altitudes) and somewhat better at higher pressures (lower altitudes). 


\subsection{High-Altitude Particles}

Another unexpected result arising from our analysis is the suggestion that large particles exist at high altitudes in Neptune's atmosphere. No such particles were evident on Uranus (Pollack et al. 1987) to the extent that could be ascertained from the lower resolution data. It is challenging to imagine ways in which the particles could achieve these large sizes on Neptune. Coagulation of small dust particles or small involatile hydrocarbon particles cannot operate quickly enough for the particles to grow to the $0.1-\mu \mathrm{m}$ radius necessary to reproduce the observations. Therefore, the particles must be large to begin with, or condensation must be occurring at high altitudes on Neptune. Large quantities of dust or ice particles of a few micrometers in radius were observed in orbit around Neptune by the Voyager 2 spacecraft (Pedersen et al. 1991, Gurnett et al. 1991). As the orbits of these particles decay, the dust will enter Neptune's atmosphere. Perhaps these particles are responsible for the observed high-altitude extinction on Neptune.

Little is known about the recondensation of ablated meteoric debris; therefore, it is difficult to estimate the possible sizes of any recondensed meteoric material; in addition, it is difficult to estimate the possible incoming flux of such material. Much of the incoming meteoroid mass examined by Moses (1992) remains unablated after being decelerated in the atmosphere. These residual meteoroids are generally between 1 and $500 \mu \mathrm{m}$ in the Moses model and could affect the extinction in Neptune's atmosphere (as well as affect condensation in the lower stratosphere) if enough of these particles exist. However, the flux of incoming debris on Neptune is not expected to greatly exceed that on Uranus (Moses 1992); thus, we would still need to explain why such particles are present on Neptune and not Uranus.

The second possibility, that of high-altitude condensation, is also difficult to analyze. We need fairly large haze production rates of $\sim 7 \times 10^{-16} \mathrm{~g} \mathrm{~cm}^{-2} \mathrm{sec}^{-1}$ at high altitudes (pressures less than $0.5 \mathrm{mbar}$ ) in order to reproduce the observations; this constraint implies the presence of a fairly abundant species that can condense above $0.5 \mathrm{mbar}$ on Neptune. No such species has yet been identified on Neptune. HCN condenses too low in the stratosphere, is too involatile, and is not abundant enough to fit the description. Perhaps water from incoming meteorites could do the trick; the flux necessary to reproduce the haze observations is within the range of incoming water flux predicted by Moses (1992). However, Lyons (1994) places slightly tighter constraints on the amount of water that is present in Neptune's stratosphere based on the lack of water absorption signatures in the Voyager UVS data. Lyons's upper limits are of the same magnitude that is needed to explain the haze observations. Again, if this process is operating, we need to explain why Neptune is different from its "twin" Uranus.

\subsection{Haze Opacity and Stratospheric Temperatures}

Figure 15 demonstrates that the stratospheric haze opacity is fairly low at visible wavelengths. This firm conclusion is relatively model independent and results directly from the observations. The total haze extinction optical depth in the stratosphere (e.g., at altitudes above 116 mbar) at visible wavelengths ranges from 0.01 to 0.03 and is more than an order of magnitude smaller at 10 mbar. The haze absorption optical depth, which is the relevant quantity required for calculations of atmospheric heating, is even smaller than the extinction optical depth since most of the observed extinction is due to scattering. Therefore, if the opacities observed at $\sim 30^{\circ} \mathrm{S}$ latitude are representative of the planet as a whole, it does not appear that the hazes can account for the heat source required to explain the stratospheric temperature inversion (see Wang and Yelle 1993). The heating calculations need to be examined in more detail.

\section{SUMMARY AND CONCLUSIONS}

An examination of the highest phase angle Voyager images has revealed many interesting properties of the stratospheric hazes on Neptune. Our principal conclusions are as follows:

1. A distinct haze layer is visible at $12_{-1}^{+1} \mathrm{mbar}$ in the $28^{\circ}-30^{\circ} \mathrm{S}$ latitude region of Neptune's stratosphere. If this haze layer is due to condensed ethane and if our adopted temperature profile is correct, then the ethane abundance at 12 mbar on Neptune is $\sim 8 \times 10^{10} \mathrm{~cm}^{-3}$, implying a volume mixing ratio at $12 \mathrm{mbar}$ of $\sim 6 \times 10^{-8}$. Condensation in this region is responsible for $\sim 70 \%$ of the total haze production in Neptune's lower stratosphere.

2. The area-weighted mean radius of the haze particles at $28 \mathrm{mbar}$, the level at which we believe we can separate the albedo-weighted phase function from the extinction coefficient in the clear-filter image, appears to be $0.13_{-0.02}^{+0.02}$ $\mu \mathrm{m}$. The haze particles must have a mean radius $\langle r\rangle_{\text {aw }}<$ $0.2 \mu \mathrm{m}$ in the lower stratosphere in order to fit the highphase-angle observations from all the different filters. These values are somewhat smaller than those inferred from the Voyager PPS observations (Pryor et al. 1992). The particle number density at $28 \mathrm{mbar}$ is $-5 \pm 3 \mathrm{~cm}^{-3}$.

3. The total atmospheric extinction in the 0.5- to 5mbar region of Neptune's stratosphere appears to significantly exceed the extinction due to Rayleigh scattering alone, suggesting the presence of high-altitude hazes. Some, but not all, of the discrepancy could be due to uncertainties in the temperature profile in this region. We 
would need to dramatically increase the average temperature in the upper troposphere and stratosphere in our model atmosphere in order to have the extinction profile more closely follow the Rayleigh curve. With the temperature profile that we have adopted, we find that the particles must have a mean radius of $0.1_{-0.02}^{+0.01} \mu \mathrm{m}$ in this pressure region in order to match the observed extinction in the middle stratosphere.

4. The high-phase-angle observations show no evidence for methane condensation in Neptune's stratosphere; however, the data are not sensitive enough in the lower stratosphere to make this a firm conclusion.

5 . The total stratospheric haze production rate inferred from our model-data comparisons is $1.0_{-0.3}^{+0.2} \times$ $10^{-15} \mathrm{~g} \mathrm{~cm}^{-2} \mathrm{sec}^{-1}$. This value is consistent with the results of the analysis of the PPS data (Pryor et al. 1992) and the analysis of ground-based data (Baines and Hammel 1994), but is considerably smaller than photochemical model predictions (Romani et al. 1993, Moses et al. 1992, and this work).

6. The rate of production of high-altitude hazes must be $7_{-2}^{+3} \times 10^{-16} \mathrm{~g} \mathrm{~cm}^{-2} \mathrm{sec}^{-1}$ if the particles are large to begin with (e.g., $\sim 0.1 \mu \mathrm{m}$ ). If the high-altitude hazes are small when they are first formed, then a comparable rate of condensation must be occurring at high altitudes in order for the particles to achieve the necessary $\sim 0.1-\mu \mathrm{m}$ mean radius by the time they fall to 0.5 mbar.

7. The optical depth of the Neptune haze ranges from 0.05 to 0.1 at visible wavelengths. Much of this opacity comes from hazes that have been transported well below the ethane condensation region. For example, optical depths at 15 mbar are much smaller, $1-4 \times 10^{-3}$. Since these opacities are small and since most of the extinction is due to scattering rather than absorption, the hazes cannot account for the stratospheric temperature inversion layer and may not contribute significantly to the heating of Neptune's stratosphere. All of the above statements assume that the $\sim 30^{\circ} \mathrm{S}$ latitude region is representative of the planet as a whole. This assumption may be incorrect.

Many of these conclusions were unanticipated before the Voyager 2 encounter with Neptune. Neptune, unlike Uranus, shows evidence for hazes at very high altitudes. Why should such hazes form on Neptune and not Uranus? What is the source of the high-altitude hazes on Neptune? The effective stratospheric haze production rate seems much smaller than it should be based on our current understanding of hydrocarbon photochemistry on the outer planets. What are the reasons for this discrepancy? The stratospheric hazes are not very dark or very abundant. What, then, is causing the strong temperature inversion in Neptune's stratosphere? Future theoretical models must address some of these unanswered questions.

\section{APPENDIX A: IMAGE PROCESSING}

The images were processed in the manner described in Rages et al. (1991) and Pollack et al. (1987). The raw images were converted to photometrically and geometrically corrected versions using software supplied by the Jet Propulsion Laboratory's Multimission Image Processing Laboratory (MIPL). Initial processing included the subtraction of dark current, correction for nonlinearity in the response function of each pixel, shading corrections to allow for nonuniform response across the vidicon, and the removal of known blemishes, reseau marks, and noise spikes. The initial data number (DN) values for each pixel were converted to specific intensity, $I / F$, where $I / F$ is defined as the ratio between the intensity of the light reflected in a given direction from a given point and the intensity of normally incident light reflecting from a nonabsorbing normally illuminated Lambert surface. To perform this conversion, we used the filter calibration corrections and adjustments discussed in Rages et al. (1991).

Although the initial encounter ephermeris (spacecraft event data record, SEDR) provided accurate positioning of the spacecraft relative to the planet's center of mass during the imaging sequences, our knowledge of the position of the scan platform in its limit cycle motion was incomplete. We refined our knowledge of the camera pointing direction and overall spacecraft/planet geometry by using an image ruavigation program that compared the observed limb prediction with the position predicted by the navigation data in the SEDR file.

This procedure was complicated by the fact that the planet is oblate and the radius of the visible limb as a function of latitude is unknown, and by the fact that only a portion of the limb and planet appears in the images. For our analysis, we assumed that the planet is an ellipsoid with an equatorial radius of $24825 \mathrm{~km}$ and a polar radius of $24400 \mathrm{~km}$. These radii were chosen to be consistent with the shape of the 1-bar geoid and with the temperature profiles derived by the radio occultation experiment (Lindal 1992) and with our estimate that the "visible" limb of the planet might be near the 35-mbar level (based on the similar situation on Uranus; see Pollack et al. 1987). Uncertainties in the planetary shape, limb radius, camera pointing, and overall viewing geometry could cause errors of $\leq 0.1^{\circ}$ in our estimates of the planetary latitudes and longitudes in the images; our estimates of the absolute limb radii might be off by $10 \mathrm{~s}$ of kilometers.

The next step in our processing of the images involved taking radial scans across the planetary limb to get $I / F$ as a function of radius at different latitudes. We started our scans well outside the limb and continued to well inside it and averaged over $30-31$ pixels in the cross-track direction for the wide-angle frames and 21 pixels for the narrow-angle frame. Some of the results of these scans are shown in Figs. A1 and A2. Figure Al shows the results for the NA-camera clear-filter high-resolution image at three different latitudes. Note that the results for the different latitudes are similar except at high altitudes where the signal quality is lower. Figure A2 shows the results for the WA-camera colored filters at the same latitude. The orange-filter scan is at a lower resolution that the others $(10-\mathrm{km}$ resolution rather than $5 \mathrm{~km})$. Remember that we assumed that the planetary limb in all the images could be found at an equivalent equatorial radius of $24825 \mathrm{~km}$. In reality, the longer wavelengths will penetrate to deeper levels than the shorter wavelengths; thus the observed "limb" of the planet will be at smaller radii (lower altitudes) for the longer wavelength images. Thus, the relative altitude scales for the different filters could be off by as much as 20 $\mathrm{km}$ or so. As already mentioned, the absolute altitude scale could also be off by 10 s of kilometers. We use theoretical models to further refine the altitude scales of the limb scans; details of this procedure can be found in Section 4.1.

Since the dark current registered by the vidicon is proportional to the vidicon temperature and since the dark current frames used in the background subtraction were not always taken at the same vidicon temperature as the image frames, we found that $I / F$ 's were generally small but 
nonzero in pixels far from the planet or well beyond the planet's terminator. In addition, some sunlight was scattered from the sun shade into the cameras at the high phase angles observed here, preventing the residual dark current from being uniform across the images. Therefore, we employed one more background-subtraction routine. For the wideangle images, we used a quadratic function in radius to calculate the average background in the "empty" or unilluminated portions of each radial scan (well off the planet or well inside the terminator). However, the terminator lay beyond the edge of the narrow-angle frame; thus, we used a constant background subtraction term for this image.

To invert the resulting $I / F$ profiles and obtain atmospheric extinction coefficient profiles, we performed a least-squares fit to the observations, using the extinction coefficient in a series of thin ellipsoidal shells and the albedo-weighted single-scattering phase function as free parameters. The ellipsoidal shells were $1 \mathrm{~km}$ thick for the NA frames and $5 \mathrm{~km}$ thick for the WA frames. The dominance of single scattering in the highphase-angle images of the stratosphere simplifies the data inversion; our inversion routine is valid so long as the slant optical depth remains less than unity.

An $I / F$ profile for a given set of free parameters was found using the method described in Rages and Pollack (1983) and Pollack et al. (1987). In this algorithm, the specific intensity due to singly scattered light in an atmosphere consisting of a series of concentric homogeneous ellipsoidal shells is calculated exactly. Light reflected from the planet and then scattered once more high in the atmosphere is included (see the method described in Pollack et al. 1987), and the angular distribution of the planet-reflected light is calculated using a matrix adding-doubling code and a scattering model of Neptune's atmosphere similar to that derived for Uranus (Rages et al. 1991). This scattering model is also used to calculate the ratio of total/single scattering as a function of scattering geometry so that the contribution from multiple scattering inside the planet's limb can be estimated. Due to the high solar phase angle of the images and the forward-scattering nature of the stratospheric haze, contributions from planet-reflected and multiply scattered light averaged only about $10-20 \%$ of the total.

Once $I / F$ and its derivatives as functions of altitude have been calculated for a given set of free parameters, singular-value decomposition (see Chap. 14 of Press et al. 1986) is used to calculate the increments in

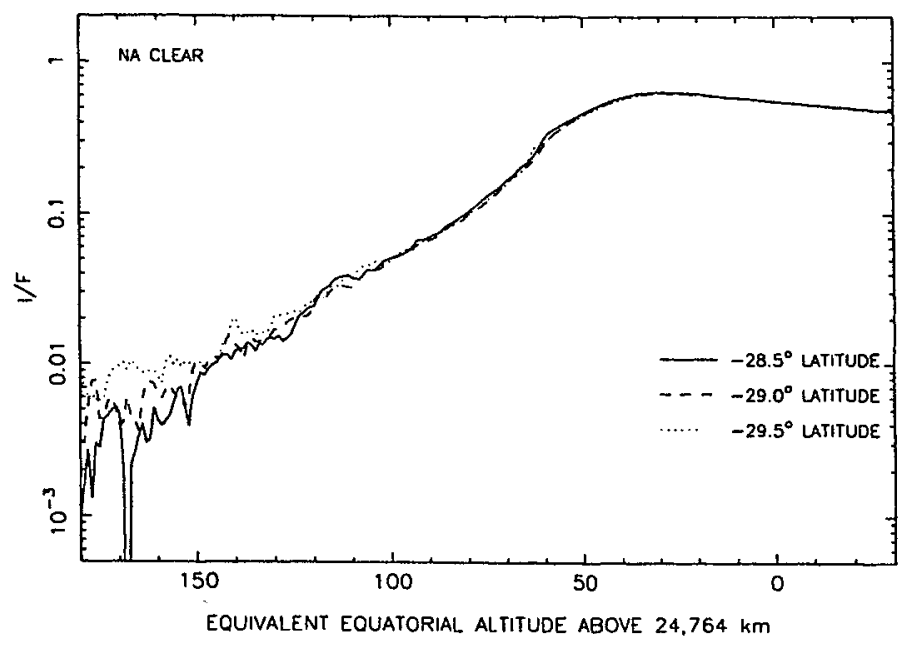

FIG. A1. Specific intensity as a function of altitude for the NAcamera clear-filter image at three different latitudes. The absolute altitude scale is uncertain due to uncertainties in the planetary shape and imaging geometry. The "bumps" in the profile near 60-65 km show evidence for a distinct haze layer in Neptune's stratosphere.

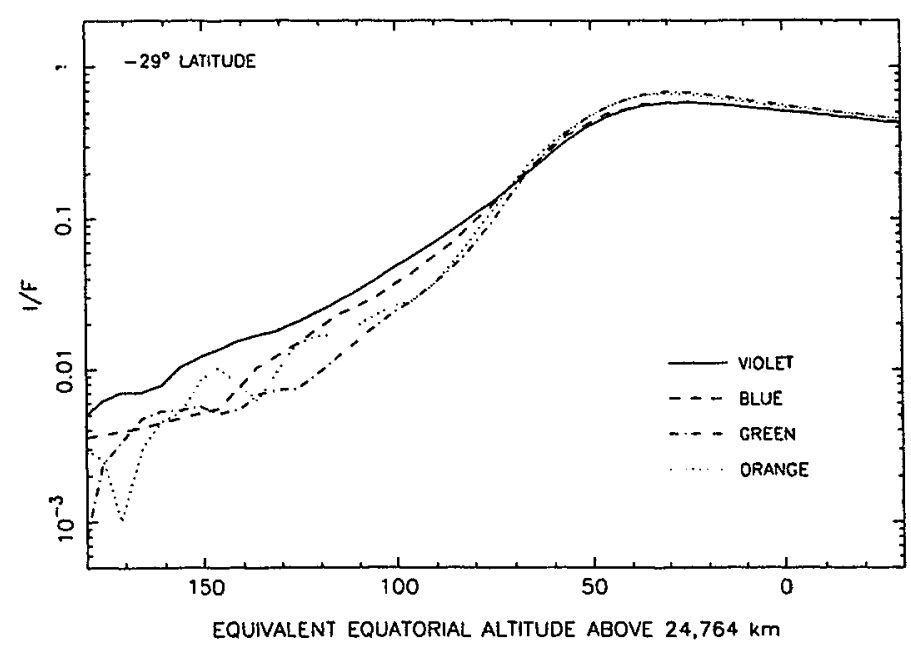

FIG. A2. Specific intensity as a function of altitude for the WAcamera colored-filter images at $29^{\circ} \mathrm{S}$ latitude. The planetary limb was assumed to be at $24825 \mathrm{~km}$ in all the images; thus the relative altitude scale between the limb scans of the different filters is not accurate. Note that these lower resolution limb scans do not notably exhibit the distinct stratospheric haze layer observed in the NA-camera dlimb scans.

these parameters that would minimize the least-squares difference between the observed and calculated $I / F$ profiles. We found it necessary to pursue this process through several iterations since the dependence of the calculated $I / F$ profile on the free parameters is nonlinear. The use of singular-value decomposition, instead of inverting the normal equation matrix directly, greatly improves the stability of the inversion process and permits a more sophisticated error analysis.

The extinction coefficients and albedo-weighted phase functions derived from the inversion routine can be compared directly with forward models of gas and aerosol scattering.

\section{APPENDIX B: MODEL HYDROSTATIC ATMOSPHERE}

Our knowledge of Neptune's thermal structure is fragmentary-detailed information only exists for certain latitudes, and altitude coverage is incomplete. To develop accurate atmospheric models, we must piece together all available observations, determine a reasonable temperature profile, and generate a background hydrostatic atmosphere that describes the temperature/density variation with altitude. Since the temperature profile appears to vary spatially across the planet, however, we must use caution when applying the observations to specific situations. Figure 2 shows some of the available information concerning Neptune's thermal structure.

The Voyager radio science (RSS) occultation data (Lindal 1992) provide accurate information concerning the tropospheric/stratospheric temperatures on Neptune from 6 bar to $\sim 0.3$ mbar. However, the RSS egress $\left(45^{\circ} \mathrm{S}\right.$ latitude) and ingress $\left(62^{\circ} \mathrm{N}\right.$ latitude) profiles differ from each other notably; furthermore, the Voyager infrared spectrometer and radiometer (IRIS) results indicate that the two latitudes probed by the radio occultations are several degrees colder than most regions of the planet (Conrath et al. 1991a). Since errors of $10 \%$ in temperature (e.g., $5 \mathrm{~K}$ at the $\sim 50-\mathrm{K}$ tropopause) can substantially affect interpretations of observations, the adoption of either of the RSS profiles would not be appropriate for an analysis of global-average ground-based observations or of Voyager observations at other latitudes. For example, Orton et al. (1992) could not reproduce the 17- to 23- $\mu \mathrm{m}$ spectra of 
Neptune as reported in Orton et al. (1990) when the RSS profiles were used in their models-Orton et al. (1992) found that they needed to perturb the RSS profiles to higher temperatures in the 500- to 1-mbar region in order to remain consistent with the infrared observations (see Fig. 2). We adopt the profile of Orton et al. (1992) to set up the background troposphere and lower stratosphere used in our analysis of the $\sim 30^{\circ} \mathrm{S}$ latitude high-phase-angle Voyager imaging data.

Since most of the observed infrared emission from the hydrocarbon gas molecules originates in Neptune's middle and upper stratosphere (1 to $10^{-4} \mathrm{mbar}$ ), it is important to determine the temperature profile in this region. Unfortunately, accurate temperature estimates are difficult to obtain in this region; e.g., the RSS and IRIS profiles do not extend to these high altitudes. Fortunately, however, the analysis of groundbased stellar occultations has met with some success (e.g., Hubbard $e t$ al. 1987, Roques et al. 1994). By analyzing a central flash event as well as the occultation data at the planetary limb, Hubbard et al. (1987) determine a temperature profile for the 0.4- to $10^{-3}$-mbar region of Neptune. A study of several Neptune stellar occultations by Roques $e t$ al. 1994 reveals that middle stratospheric temperatures on Neptune might vary considerably with location on the planet and perhaps with time as well. Roques et al. derive profiles for the upper stratosphere that typically fall within a temperature range of $140-200 \mathrm{~K}$; the Hubbard et al. profile also lies in this range.

Another useful piece of information for determining the temperature profile in Neptune's middle and upper stratosphere is the measurement of the hydrogen density as a function of altitude in the $\sim 150-$ to $300-\mathrm{km}$ altitude region $\left(\sim 1.4\right.$ to $0.064 \mathrm{mbar}$ in our model) at $45^{\circ} \mathrm{S}$ and $61^{\circ} \mathrm{N}$ by the Voyager ultraviolet spectrometer (Yelle et al. 1993). The $\mathrm{H}_{2}$ density in this region is estimated from the observed absorption at wavelengths greater than $1500 \AA$. Any pressure-temperature profile adopted for the hydrostatic model must reproduce the observed $\mathrm{H}_{2}$ densities at these altitudes.

We have constructed a temperature profile for Neptune's middle and upper stratosphere based on the Hubbard et al. (1987) central flash data at $\sim 0.37$ mbar, on some of the profiles of Roques et al. (1994), on the information from Yelle et al. (1993), and on the ground-based infrared spectral analysis of Orton et al. (1992). Orton et al. use both high- and low-resolution infrared spectra of $\mathrm{CH}_{3} \mathrm{D}$ and $\mathrm{CH}_{4}$ to derive a globally averaged temperature of $168 \pm 10 \mathrm{~K}$ in the $10^{-2}$ - to $10^{-3}$-mbar region. This $168-\mathrm{K}$ value is compelling for other reasons: The stratospheric methane mixing ratio implied from Orton et al.'s ground-based IR spectra at this temperature compares well with the results form the UVS analysis (Yelle et al. 1993), $168 \mathrm{~K}$ is near the center of the stellar occultation profiles discussed above (Roques et al. 1994), and this choice of temperature is consistent with the other Orton $e t$ al. data sets.

The temperature in the thermosphere of Neptune has little effect on the photochemistry (Moses 1991). However, much of the useful UVS data extends to these high altitudes; therefore, it is important to have some knowledge of the atmospheric density variation with altitude (i.e. the temperature profile) in this region. We adopt the theoretical profile of Wang and Yelle (1992), which was developed to match the shortwavelength UVS spectra. One useful piece of information comes from the measurement of the $\mathrm{H}_{2}$ density at $\sim 700 \mathrm{~km}$ above $\mathrm{I}$ bar derived from the UVS analysis of absorption in the $800-$ to $1100-\AA \mathrm{H}_{2}$ electronic band systems (Yelle et al. 1993). If the temperature profile in the lower and middle stratosphere is incorrect, the predicted altitude for the observed density could be off by as much as 10 s of kilometers, and the model results would never compare well with the UVS lightcurves.

Establishing a temperature profile is only part of the process of deriving a background atmosphere. The density variation with altitude (and latitude) also depends on the planetary shape, the gravitational field, and the atmospheric mean molecular mass as a function of altitude or pressure. The planetary shape is obtained from the RSS data (Lindal 1992). The variation of gravitational acceleration with altitude is derived as in Lindal et al. (1985) with information on $J_{2}, J_{4}, G M$, and rotation rate from Lindal (1992) and Lindal et al. (1990). Some uncertainties arise in the gravity profile (especially at midlatitudes) due to our lack of knowledge of the wind speeds at all altitudes and latitudes. Hammel $e t$ al. (1989) and Limaye and Sromovsky (1991) track cloud features about the planet and determine cloud-top wind speeds as a function of latitude on Neptune. Wind speeds might decay with height, however. We therefore use a constant rotation rate of $16.1 \mathrm{hr}$, the interior rotation rate as measured by the magnetic field, at all latitudes. The mean molecular mass of the atmosphere drops off with altitude in the middle and upper stratosphere as helium and other species heavier than hydrogen become diffusively separated. We estimate the altitude variation of the mean molecular mass using preliminary photochemistry and diffusion models. Small errors in the planetary shape, gravitational field, mean molecular mass, or the estimated thermal profile can lead to measurable errors in the atmospheric density predicted at specific altitudes and can cause problems during the comparison of the models with the various observations.

Figure 3 shows the resulting variation of hydrogen number density with altitude (or pressure in this case) compared with the UVS egress occultation results presented by Yelle et al. (1993). The calculations were performed for $49^{\circ}$ latitude to facilitate the comparison. The calculations compare fairly well with observations. Unfortunately, we have no measurements of the hydrogen density in the middle and upper stratosphere, precisely in the region in which temperature measurements are difficult to obtain; therefore, our atmospheric density profile in this region is uncertain.

\section{ACKNOWLEDGMENTS}

Damon Simonelli and W. Reid Thompson assisted in the early processing of the images, and Shelly Pope wrote the preliminary version of the aerosol-dynamics code. We thank $O$. Brian Toon, Anthony Jones, Kevin Baines, and an anonymous reviewer for useful comments and suggestions. This research was supported in part by the National Research Council Associateship Program, by NASA grants from the NDAP program (889-59-01-28) and Planetary Atmospheres Program (RTOP 154-10-80-01), and by the Lunar and Planetary Institute, which is operated by the Universities Space Research Association under NASA Contract NASW-4574. This paper represents Contribution 843 of the Lunar and Planetary Institute.

\section{REFERENCES}

Allen, M., Y. L. Yung, and G. R. Gladstone 1992. The relative abundance of ethane to acetylene in the Jovian stratosphere. Icarus 100, 527-533. See also Icarus 104, 155.

Allen, M., Y. L. YUNG, AND J. W. WATERS 1981. Vertical transport and photochemistry in the terrestrial mesosphere and lower thermosphere (50-120 km). J. Geophys. Res. 86, 3617-3627.

Atreya, S. K., AND J. J. Ponthieu 1983. Photolysis of methane and the ionosphere of Uranus. Planet. Space Sci. 31, 939-944.

Atreya, S. K., AND P. N. Romani 1985. Photochemistry and clouds of Jupiter, Saturn, and Uranus. In Recent Advances in Planetary Meteorology (G. E. Hunt, Ed.), pp. 17-68. Cambridge Univ. Press, Cambridge.

Atreya, S. K., B. R. Sandel, ANd P. N. Romani 1991. Photochemistry and vertical mixing. In Uranus (J. T. Bergstralh, E. D. Miner, and M. Shapley Matthews. Eds.), pp. 110-146. Univ. of Arizona Press, Tucson.

Baines, K. H., AND H. B. Hammel 1994. Clouds, hazes, and the 
stratospheric methane abundance in the atmosphere of Neptune. Icarus 109, 20-39.

Baines, K. H., H. B. Hammel, K. A. Rages, P. N. Romani, and R. E. SAMUelson 1994. Clouds and hazes in the atmosphere of Neptune. In Neptune and Triton (D. Cruikshank, Ed.). Univ. of Arizona Press, Tucson. In press.

Bezard, B., P. N. Romani, B. J. Conrath, and W. C. Maguire 1991. Hydrocarbons in Neptune's atmosphere from Voyager infrared observations. J. Geophys. Res. 96, 18,961-18,976.

Bishop, J., S. K. Atreya, P. N. Romani, B. R. SANdel, ANd F. Herbert 1992. Voyager 2 Ultraviolet Spectrometer solar occultations at Neptune: Constraints on the abundance of methane in the stratosphere. J. Geophys. Res. 97, 11,681-11,694.

Bishop, J., S. K. Atreya, F. Herbert, and P. N. Romani 1990. Reanalysis of Voyager 2 UVS occultations at Uranus: Hydrocarbon mixing ratios in the equatorial stratosphere. Icarus 88, 448-464.

Broadfoot, A. L., S. K. Atreya, J. L. Bertaux, J. E. Blamont, A. J. Dessler, T. M. Donahue, W. T. Forrester, D. T. Hall, F. Herbert, J. B. Holberg, D. M. Hunten, V. A. Krasnopolsky, S. Linick, J. I. Lunine, J. C. McConnell, H. W. Moos, B. R. SANDel, N. M. Schneider, D. E. Shemansky, G. R. Smith, D. F. STROBEL, AND R. V. YelLe 1989. Ultraviolet spectrometer observations of Neptune and Triton. Science 246, 1459-1466.

Caldwell, J., R. WAGener, AND K.-H. Fricke 1988. Observations of Neptune and Uranus below $2000 \AA$ with the IUE. Icarus 74, 133-140.

Conrath, B. J., F. M. Flasar, and P. J. Gierasch 1991a. Thermal structure and dynamics of Neptune's atmosphere from Voyager measurements. J. Geophys. Res. 96, 18,931-18,939.

Conrath, B. J., D. Gautier, G. F. Lindal, R. E. Samuelson, and W. A. SCHAFFER 1991b. The helium abundance of Neptune from Voyager measurments. J. Geophys. Res. 96, 18,907-18,919.

Danielson, G. E., P. N. Kupferman, T. V. Johnson, and L. A. SODERBLOM 1981. Radiometric performance of the Voyager cameras. J. Geophys. Res. 86, 8683-8689.

Gladstone, G. R., M. Allen, and Y. L. Yung 1994. Hydrocarbon photochemistry in the upper atmosphere of Jupiter. Submitted.

Gurnett, D. A., W. S. Kurth, L. J. Granroth, and S. C. AllenDORF 1991. Micron-sized particles detected near Neptune by the Voyager 2 plasma wave instrument. J. Geophys. Res. 96, 19,17719,186 .

HAMMEL, H. B. 1989. Neptune cloud structure at visible wavelengths. Science 244, 1165-1167.

Hammel, H. B., R. F. Beebe, E. M. DeJong, C. J. Hansen, C. D. Howell, A. P. Ingersoll, T. V. Johnson, S. S. Limaye, J. A. Magelhäes, J. B. Pollack, L. A. Sromovsky, V. E. Suomi, AND C. E. SwIFT 1989. Neptune's wind speeds obtained by tracking clouds in Voyager images. Science 245, 1367-1369.

Herbert, F., B. R. Sandel, R. V. Yelle, J. B. Holberg, A. L. BroAdFoot, AND D. E. SHEMANSKy 1987. The upper atmosphere of Uranus: EUV occultations observed by Voyager 2. J. Geophys. Res. 92, 15,093-15,109.

Hubbard, W. B., P. D. Nicholson, E. Lellouch, B. Sicardy, A. Brahic, F. Vilas, P. Bouchet, R. A. Mclaren, R. L. Millis, L. H. Wasserman, J. H. Elias, K. Matthews, and J. D. McGill 1987. Oblateness, radius, and mean stratospheric temperature of Neptune from the 1985 August 20 occultation. Icarus 72, 635-646.

Khare, B. N., C. Sagan, W. R. Thompson, E. T. Arakawa, and P. VoTAW 1987. Solid hydrocarbon aerosols produced in simulated Uranian and Neptunian stratospheres. J. Geophys. Res. 92, 1506715082.

Kostiuk, T. F., P. Romani, F. Espenak, and B. Bezard 1992.
Stratospheric ethane on Neptune: Comparison of Groundbased and Voyager IRIS retrievals. Icarus 99, 353-362.

Lane, A. L., R. A. West, C. W. Hord, R. M. Nelson, K. E. SiMMONS, W. R. PryoR, L. W. Esposito, L. J. HoRN, B. D. Wallis, B. J. Buratti, T. G. Brophy, P. Yanamandra-Fisher, J. E. ColWell, D. A. Bliss, M. J. Mayo, AND W. D. Smythe 1989. Photometry from Voyager 2: Initial results from the Neptunian atmosphere, satellites, and rings. Science 246, 1450-1454.

LEE, Y. T., M. ALLEN, AND Y. L. YUNG 1993. Solar system hydrocarbon photochemistry: Impact of new $\mathrm{CH}_{4} \mathrm{Ly} \alpha$ photodissociation quantum yields. Bull. Am. Astron. Soc. 25, 1035.

LIMAYE, S. J., AND L. A. SromovsKy 1991. Winds of Neptune: Voyager observations of cloud motions. J. Geophys. Res. 96, 18,94118,960 .

LiNDAL, G. F. 1992. The atmosphere of Neptune: An analysis of radio occultation data acquired with Voyager 2. Astron. J. 103, 967-982.

Lindal, G. F., J. R. Lyons, D. N. Sweetnam, V. R. Eshleman, D. P. Hinson, AND G. L. TYler 1990. The atmosphere of Neptune: Results of radio occultation measurements with the Voyager 2 spacecraft. Geophys. Res. Lett. 17, 1733-1736.

Lindal, G. F., D. N. Sweetnam, and V. R. Eshleman 1985. The atmosphere of Saturn: An analysis of the Voyager Radio occultation measurements. Astron. J. 90, 1136-1146.

LINDZEN, R. S. 1971. Tides and gravity waves in the upper atmosphere. In Mesopheric Models and Related Experiments (G. Fiocco, Ed.), pp. 122-130. Reidel, Dordrecht, the Netherlands.

LYoNs, J. R. 1994. Constraints on meteoroidal influx into the upper atmospheres of Uranus and Neptune. Submitted.

Mordaunt, D. H., I. R. Lambert, G. P. Morley, M. N. R. Ashford, R. N. Dixon, AND C. M. Western 1993. Primary product channels in the photodissociation of methane at $121.6 \mathrm{~nm}$. J. Chem. Phys. 98, 2054-2065.

MosEs, J. 1. 1992. Meteoroid ablation in Neptune's atmosphere. Icarus 99, 368-383.

Moses, J. I. 1991. I. Phase Transformations and the Spectral Reflectance of Solid Sulfur: Possible Metastable Sulfur Allotropes on Io's Surface. II. Photochemistry and Aerosol Formation in Neptune's Atmosphere. Ph.D. thesis, California Institute of Technology, Pasadena.

Moses, J. I., M. Allen, AND Y. L. Yung 1992. Hydrocarbon nucleation and aerosol formation in Neptune's atmospere. Icarus 99, 318346.

Orton, G., K. H. Baines, J.' Caldwell, P. Romani, A. T. ToKUNAGA, AND R. A. WEST 1990. Calibration of the 7- to 14- $\mu \mathrm{m}$ brightness spectra of Uranus and Neptune. Icarus 85, 257-265.

Orton, G. S., J. H. Lacy, J. M. Achtermann, P. Parmar, and W. E. Blass 1992. Thermal spectroscopy of Neptune: The stratospheric temperature, hydrocarbon abundances, and isotopic ratios. Icarus 100, 541-555.

Pedersen, B. M., N. Meyer-Vernet, M. G. Aubier, and P. Zarka 1991. Dust distribution around Neptune: Grain impacts near the ring plane measured by the Voyager planetry radio astronomy experiment. J. Geophys. Res. 96, 19,187-19,196.

Pollack, J. B., K. Rages, S. K. Pope, M. G. Tomasko, P. N. RoMANI, AND S. K. ATREYA 1987. Nature of the stratospheric haze on Uranus: Evidence for condensed hydrocarbons. J. Geophys. Res. 92, 15037-15065.

Press, W. H., B. P. Flannery, S. A. Teukolsky, and W. T. VetTERLING 1986. Numerical Recipes. Cambridge Univ. Press, Cambridge.

Pryor, W. R., R. A. West, K. E. Simmons, and M. Delitsky 1992. 
High-phase-angle observations of Neptune at 2650 and $7500 \AA$ : Haze structure and particle propeties. Icarus 99, 302-317.

RAGES, K., AND J. B. PolLACK 1983. Vertical distribution of scattering hazes in Titan's upper atmosphere. Icarus 55, 50-62.

Rages, K., J. B. Pollack, M. G. Tomasko, and L. R. Doose 1991. Properties of scatterers in the troposphere and lower stratosphere of Uranus based on Voyager imaging data. Icarus 89, 359-376.

RizK, B., D. M. HuNTEN, AND S. ENGEL 1991. Effects of size-dependent emissivity on maximum temperatures during micrometeorite entry. J. Geophys. Res. 96, 1303-1314.

Romani, P. N., AND S. K. ATreya 1988. Methane photochemistry and haze production on Neptune. Icarus $\mathbf{7 4}$, 424-445.

RomANi, P. N., AND S. K. ATREYA 1989. Stratospheric aerosols from $\mathrm{CH}_{4}$ photochemistry on Neptune. Geophys. Res. Lett. 16, 941-944.

Romani, P. N., J. Bishop, B. Bézard, and S. K. Atreya 1993. Methane photochemistry on Neptune: Ethane and acetylene mixing ratios and haze production. Icarus 106, 442-463.

Roques, F., B. SiCARDY, AND A. BRahic 1994. Neptune's upper stratosphere, 1983-1990. Ground-based stellar occultation observations III. Temperature profiles. Astron. Astrophys. 288, 985-1011.

Smith, B. A., L. A. Soderblom, D. Banfield, C. Barnet, A. T. Basilevsky, R. F. Beebe, K. Bollinger, J. M. BoyCe, A. Brahic, G. A. Briggs, R. H. Brown, C. Chyba, S. A. Collins, T. Colvin, A. F. Cook II, D. Crisp, S. K. Croft, D. Cruikshank, J. N. Cuzzi, G. E. Danielson, M. E. Davies, E. De Jong, L. Dones, D. Godfrey, J. Goguen, I. Grenier, V. R. Haemmerle, H. Hammel, C. J. Hansen, C. P. Halfenstein, C. Howell, G. E. Hunt, A. P. Ingersoll, T. V. Johnson, J. Kargel, R. Kirk, D. I. Kuehn, S. Limaye, H. Masursky, A. McEwen, D. Morrison, T. Owne, W. Owne, J. B. Pollack, C. C. Porco, K. Rages, P. Rogers, D. Rudy, C. Sagan, J. Schwartz, E. M. Shoemaker, M. Showalter, B. Sicardy, D. Simonelli, J. Spencer, L. A. Stro-
Movksy, C. Stoker, R. G. Strom, V. E. SUOMi, S. P. Synott, R. J. Terrile, P. Thomas, W. R. Thompson, A. VerbisCer, AND J. VEVERKA 1989 . Voyager 2 at Neptune: Imaging science results. Science 246, 1422-1449.

STONE, P. H. 1976. The meteorology of the Jovian atmosphere. In Jupiter (T. Gehrels, Ed.), pp. 586-618. Univ. of Arizona Press, Tucson.

Summers, M. E., AND D. F. Strobel 1989. Photochemistry of the atmosphere of Uranus. Astrophys. J. 346, 495-508.

TOON, O. B., AND T. P. ACKERMAN 1981. Algorithm for the calculation of scattering by stratified spheres. Appl. Optics 20, 3657-3660.

Toon, O. B., R. P. Turco, AND J. B. Pollack 1980. A physical model of Titan's clouds. Icarus 43, 260-282.

Turco, R. P., P. Hamill, O. B. Toon, R. C. Whitten, and C. S. KIANG 1979. The NASA-Ames Research Center stratospheric aerosol model. I. Physical processes and computational analogs. NASA Technical Paper 1362.

VAN DE HULST, H. C. 1957. Light Scattering by Small Particles, pp. 70-84. Dover, New York.

WANG, Y., AND R. V. YELLE 1992. Models of the thermal profile of Neptune's upper atmosphere. In Neptune and Triton Conference Proceedings, Tucson, Az, p. 92.

WANG, Y., AND R. V. YELLE 1993. Modeling the thermal structure of Neptune's stratosphere. Bull. Am. Astron. Soc. 25, 1078.

West, R. A., K. H. Baines, and J. B. Pollack 1991. Clouds and aerosols in the Uranian atmosphere. In Uranus (J. T. Bergstralh, E. D. Miner, and M. Shapley Matthews, Eds.), pp. 296-324. Univ. of Arizona Press, Tucson.

Yelle, R. V., F. Herbert, B. R. SANdel, R. J. VerVACK, JR., ANd T. WENTZEL 1993. The distribution of hydrocarbons in Neptune's upper atmosphere. Icarus 104, 38-59. 